\title{
Total solid-phase synthesis of dehydroxy fengycin
}

\section{derivatives}

Cristina Rosés, Cristina Camó, Àngel Oliveras, Lluis Moll, Nerea López,

Lidia Feliu,* and Marta Planas*

LIPPSO, Department of Chemistry, University of Girona, Maria Aurèlia Capmany 69, 17003 Girona, Spain

${ }^{*}$ Corresponding authors

E-mail: lidia.feliu@udg.edu

marta.planas@udg.edu 


\section{Table of Contents/Abstract Graphic}

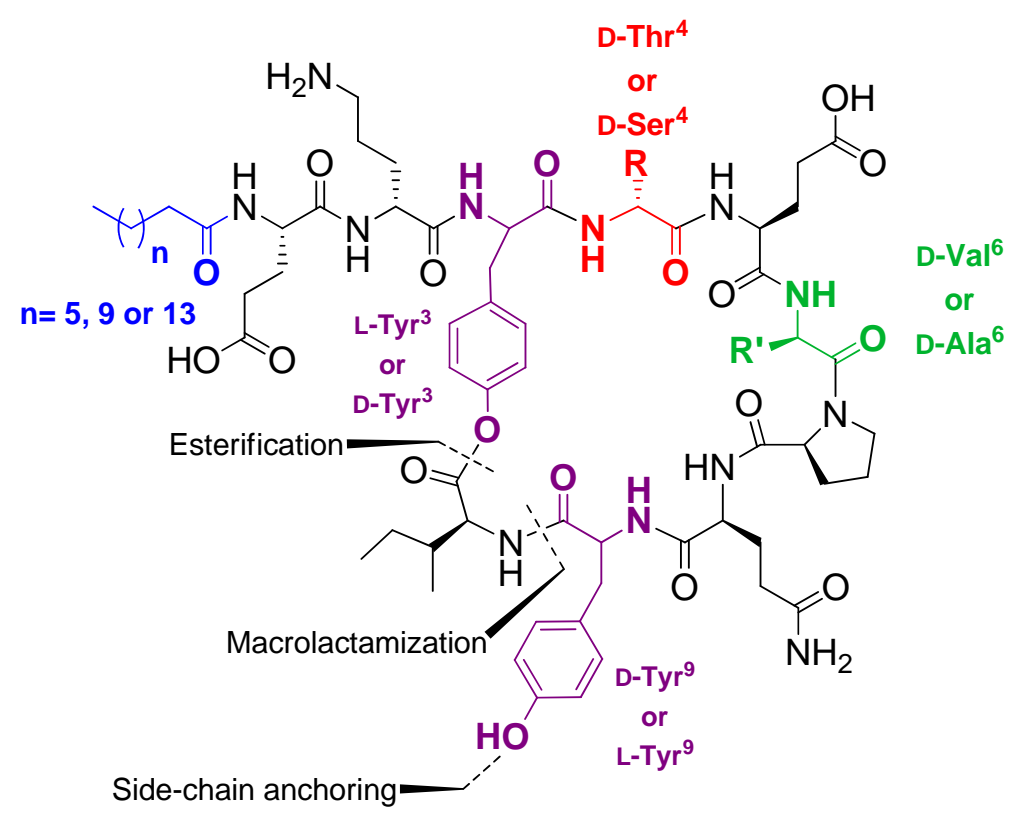




\section{Abstract}

A rapid and efficient solid-phase strategy for the synthesis of dehydroxy fengycins derivatives is described. This synthetic approach involved the linkage of a Tyr to a Wang resin via a Mitsunobu reaction and the elongation of the peptide sequence followed by subsequent acylation of the $\mathrm{N}$-terminus of the resulting linear peptidyl resin, esterification of the phenol group of a Tyr with an Ile, and final macrolactamization. The amino acid composition as well as the presence of the $\mathrm{N}$-terminal acyl group significantly influenced the stability of the macrolactone. Cyclic lipodepsipeptides with a L-Tyr ${ }^{3} / \mathrm{D}-\mathrm{Tyr}^{9}$ configuration were more stable than those containing the Tyr residues with an opposite configuration. This work constitutes the first approach on the total solid-phase synthesis of dehydroxy fengycin derivatives. 


\section{Introduction}

Cyclic lipodepsipeptides are a structurally diverse group of nonribosomal metabolites typically produced by bacterial and fungal species, such as Pseudomonas or Bacillus strains. ${ }^{1-4}$ This class of compounds have raised interest due to their wide range of biological activities and to their structural complexity. The main structural feature of these cyclic peptides is the occurrence of an ester bond between the side-chain of an amino acid and the C-terminal end of the sequence. The fatty acid moiety is commonly attached to the N-terminus of this macrolactone. Moreover, the complexity of the structure of these lipodepsipeptides is increased by the combination of L- and D-amino acids as well as of other unnatural residues present in their sequence. ${ }^{5,6}$

Fengycins are a family of natural cyclic lipodepsipeptides isolated from Bacillus strains. ${ }^{5,6}$ They consist of an eight-residue macrocycle with a phenyl ester linkage between the phenol group of a tyrosine (Tyr) and the $\alpha$-carboxylic group of an isoleucine (Ile) (Figure 1). This cyclic depsipeptide bears at its $\mathrm{N}$-terminus a dipeptidyl tail acylated with a $\beta$-hydroxy fatty acid. Different fengycin isoforms have been described, being fengycins $\mathrm{A}$ and $\mathrm{B}$ the most common. ${ }^{7-14}$ These two isoforms differ on the amino acid at position 6 , which is a D-alanine (D-Ala) in fengycin A and a D-valine (DVal) in the B isoform (Figure 1). Sang-Cheol et al. isolated from Bacillus amyloliquefaciens fengycin S. ${ }^{15}$ Compared to fengycin B, this isoform bears a D-serine (D-Ser) at position 4 instead of a D-allothreonine (D-allo-Thr). The configuration of Tyr residues at position 3 and 9 of fengycins has been a controversial issue. Fengycins were first reported to incorporate a D-Tyr ${ }^{3}$ and an L-Tyr ${ }^{9}$ whereas the opposite configuration ( $\left.\mathrm{L}-\mathrm{Tyr}^{3} / \mathrm{D}-\mathrm{Tyr}^{9}\right)$ was assigned to another family of related peptides named as plipastatins. ${ }^{7,16}$ However, it has been recently confirmed that the configuration of $\mathrm{Tyr}^{3}$ and $\mathrm{Tyr}^{9}$ in fengycins and plipastatins is identical, being L-Tyr ${ }^{3} / \mathrm{D}-\mathrm{Tyr}^{9} .{ }^{17-20}$ Since the term fengycins is most 
widely used than plipastatins, herein we employed the former term for the peptides of this study, but using the correct configuration.

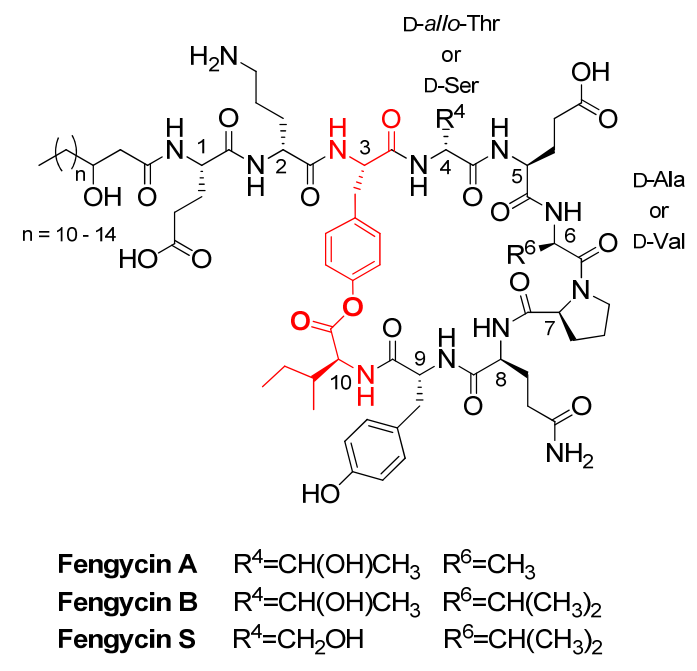

Figure 1. Structure of fengycins A, B and S.

Fengycin lipopeptides have attracted considerable interest due to their strong antifungal activity and their low hemolysis compared to other lipopeptides isolated from Bacillus spp. ${ }^{7,21,22}$ Their significant antifungal activity has been ascribed to their propensity to form stable membrane-bound aggregates. ${ }^{23}$ Moreover, they have been described as elicitors of plant defence responses. ${ }^{24,25}$ The use of compounds with such activity is considered as a promising alternative approach for the protection of crops against phytopathogens. . 26,27

Despite their interest, up to now and to the best of our knowledge, the synthesis of fengycins has only been accomplished by the group of Marahiel and Essen. Their strategy comprised the solid-phase preparation of the linear precursor and its enzymatic cyclization in solution. ${ }^{5,28,29}$ The lack of suitable synthetic protocols for fengycins could be attributed to the presence of a highly labile phenyl ester function in their structure. In fact, the synthesis of cyclic depsipeptides, either in solution or on solid 
phase, has only been reported for those peptides bearing an ester bond involving the $\beta$-hydroxyl group of a Ser or a Thr residue. ${ }^{30-34}$ Recently, we have established a convenient solid-phase strategy for the preparation of the macrolactone of eight amino acids present in fengycins. ${ }^{35}$ The key steps of the synthesis were the formation of the phenyl ester bond and the on-resin head-to-side-chain cyclization.

In view of these previous results, we decided to study the application of this methodology to the total solid-phase synthesis of dehydroxy fengycin derivatives with the general structure I (Figure 2). These derivatives are fengycin A, B and S analogues that contain a D-Thr ${ }^{4}$ instead of a D-allo-Thr ${ }^{4}$ and a dehydroxy fatty acid chain. Synthesis of analogues containing both an L-Tyr ${ }^{3} / \mathrm{D}-\mathrm{Tyr}^{9}$ or a D-Tyr ${ }^{3} / \mathrm{L}-$ Tyr $^{9}$ configuration was studied. This approach would benefit from the advantages inherent to the solidphase methodology and would allow rapid access to a variety of fengycin analogues.

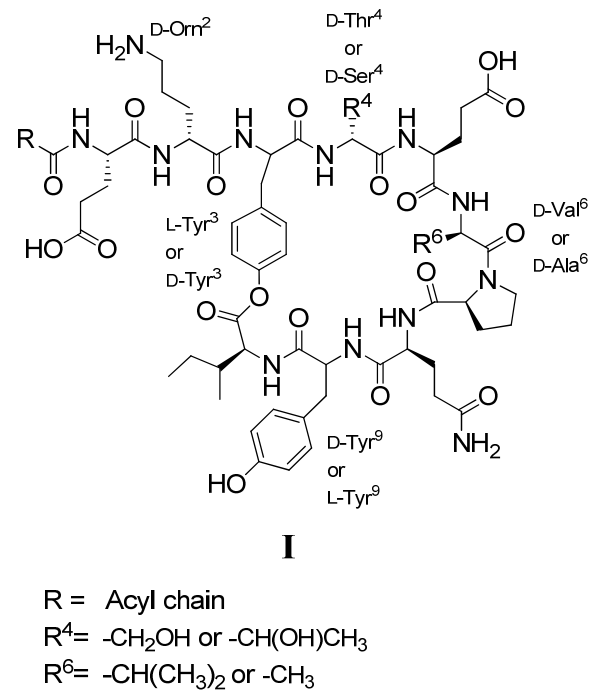

Figure 2. General structure of dehydroxy fengycin derivatives I. 


\section{Results and Discussion}

\section{Synthesis of Cyclic Lipodepsipeptides Bearing a L-Tyr ${ }^{3} / \mathrm{D}^{-T y r}{ }^{9}$}

Our investigations started with the solid-phase synthesis of the cyclic lipodepsipeptide BPC838 containing D-Thr ${ }^{4}, \mathrm{D}-\mathrm{Val}^{6}, \mathrm{D}-\mathrm{Tyr}^{9}$ and an octanoyl group (Scheme 1). This synthesis was chosen as a model to check the feasibility of our approach. Based on our previous experience on the preparation of cyclic depsipeptides, the retrosynthetic route that we envisaged involved as main steps: (i) the preparation of the heptapeptidyl resin 1 with the N-terminal ${ }_{\text {L-Tyr }}{ }^{3}$ protected with a $p$ nitrobenzyloxycarbonyl ( $p \mathrm{NZ}$ ) group, (ii) the ester bond formation between $\mathrm{L}-\mathrm{Tyr}^{3}$ and $\mathrm{Ile}{ }^{10}$, (iv) the simultaneous deprotection of $\mathrm{Ile}^{10}$ and ${ }_{\mathrm{D}}-\mathrm{Tyr}^{9}$ followed by the on-resin cyclization and (vi) the elongation of the peptide sequence and its final acylation. As protecting groups, allyl (All) and allyloxycarbonyl (Alloc) were selected for $\mathrm{Tyr}^{9}$ and $\mathrm{Ile}^{10}$, respectively, because they can be simultaneously removed in presence of palladium and are orthogonal with the 9fluorenylmethoxycarbonyl (Fmoc) and tert-butyl $\left({ }^{t} \mathrm{Bu}\right)$ groups. The $p \mathrm{NZ}$ group was chosen to protect $\mathrm{Tyr}^{3}$ because it has been reported to be orthogonal to Fmoc as well as to ${ }^{t} \mathrm{Bu}$ and allyl-based protecting groups, and its removal can be achieved under neutral conditions using $\mathrm{SnCl}_{2}$ in presence of catalytic amounts of acid. ${ }^{36}$ 


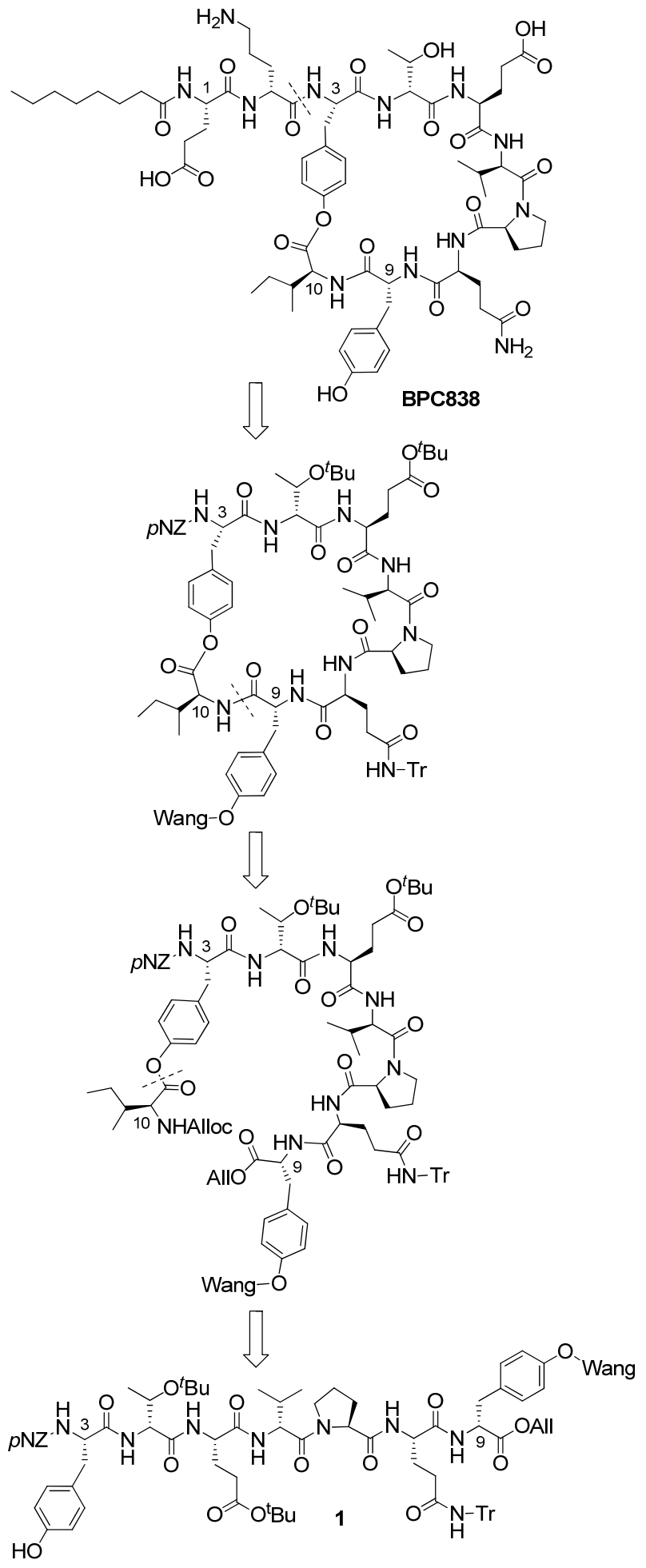

Scheme 1. Retrosynthetic analysis of BPC838. 
According to this route, Fmoc-D-Tyr-OAll was anchored to a Wang resin by treatment with $\mathrm{PPh}_{3}$ and diisopropylazodicarboxylate (DIAD) in anhydrous tetrahydrofuran (THF) under microwave irradiation for $30 \mathrm{~min}$ at $60^{\circ} \mathrm{C}$ (Scheme 2). ${ }^{35}$ The loading of the resulting resin Fmoc-D-Tyr(Wang)OAll was $0.33 \mathrm{mmol} / \mathrm{g}$ as determined by the Fmoc method. ${ }^{37}$ In addition a Marfey's test confirmed that the D-Tyr residue did not racemise under these conditions. ${ }^{38}$ Then, the peptidyl resin $p$ NZ-Tyr ${ }^{3}-\mathrm{D}-$ $\operatorname{Thr}\left({ }^{t} \mathrm{Bu}\right)-\mathrm{Glu}\left(\mathrm{O}^{t} \mathrm{Bu}\right)-\mathrm{D}-$ Val-Pro-Gln$(\mathrm{Tr})-\mathrm{D}-\mathrm{Tyr}^{9}($ Wang)-OAll (1) was prepared following a Fmoc $/{ }^{t} \mathrm{Bu} /$ allyl (All) strategy. Fmoc group removal was performed using piperidine/DMF (3:7) and the coupling of the corresponding amino acids was mediated by $N, N^{\prime}$-diisopropylcarbodiimide (DIPCDI) and 2-cyano-2-(hydroxyimino)acetate (Oxyma) in DMF. All the amino acids, except for Tyr ${ }^{3}$, were incorporated as Fmoc-derivatives with the side-chain group protected with trityl (Tr) for Gln and ${ }^{t} \mathrm{Bu}$ for Glu and Thr. $\mathrm{Tyr}^{3}$ was introduced as $p \mathrm{NZ}-\mathrm{Tyr}-\mathrm{OH}$ to allow the required esterification of the phenol group. This tyrosine derivative was prepared from commercially available $\mathrm{H}-\mathrm{Tyr}\left(\mathrm{O}^{t} \mathrm{Bu}\right)-\mathrm{OH}$ by treatment with $p$ NZ-N ${ }_{3}{ }^{36}$ followed by side-chain deprotection under acidic conditions $(68 \%$ overall yield). After completion of the peptide sequence, acidolytic cleavage with trifluoroacetic acid (TFA)/ $\mathrm{H}_{2} \mathrm{O} /$ triisopropylsilane (TIS) $(95: 2.5: 2.5)$ of an aliquot of resin 1 afforded the expected peptide $p$ NZ-Tyr-D-Thr-Glu-D-Val-Pro-Gln-D-Tyr-OAll in 65\% HPLC purity.

Then, Alloc-Ile-OH was incorporated to $\mathbf{1}$ using the conditions previously described for the preparation of cyclic depsipeptides analogous to the cyclic moiety of fengycins (Scheme 2). ${ }^{35}$ Therefore, esterification of $\mathbf{1}$ with Alloc-Ile-OH was achieved employing DIPCDI, 4dimethylaminopiridine (DMAP) and N,N-diisopropylethylamine (DIEA) in DMF. Two treatments of $24 \mathrm{~h}$ were necessary to obtain a complete conversion. An aliquot of the resulting resin $p \mathrm{NZ}-\mathrm{Tyr}^{3}(\mathrm{O}-$ $\mathrm{Ile}^{10}$-Alloc)-D-Thr( $\left({ }^{t} \mathrm{Bu}\right)-\mathrm{Glu}\left(\mathrm{O}^{t} \mathrm{Bu}\right)-\mathrm{D}-$ Val-Pro-Gln(Tr)-D-Tyr ${ }^{9}(\mathrm{Wang})-\mathrm{OAll}$ (2) was treated under acidolytic conditions yielding the expected linear depsipeptide $p$ NZ-Tyr(O-Ile-Alloc)-D-Thr-Glu-DVal-Pro-Gln-D-Tyr-OAll in 87\% HPLC purity. 

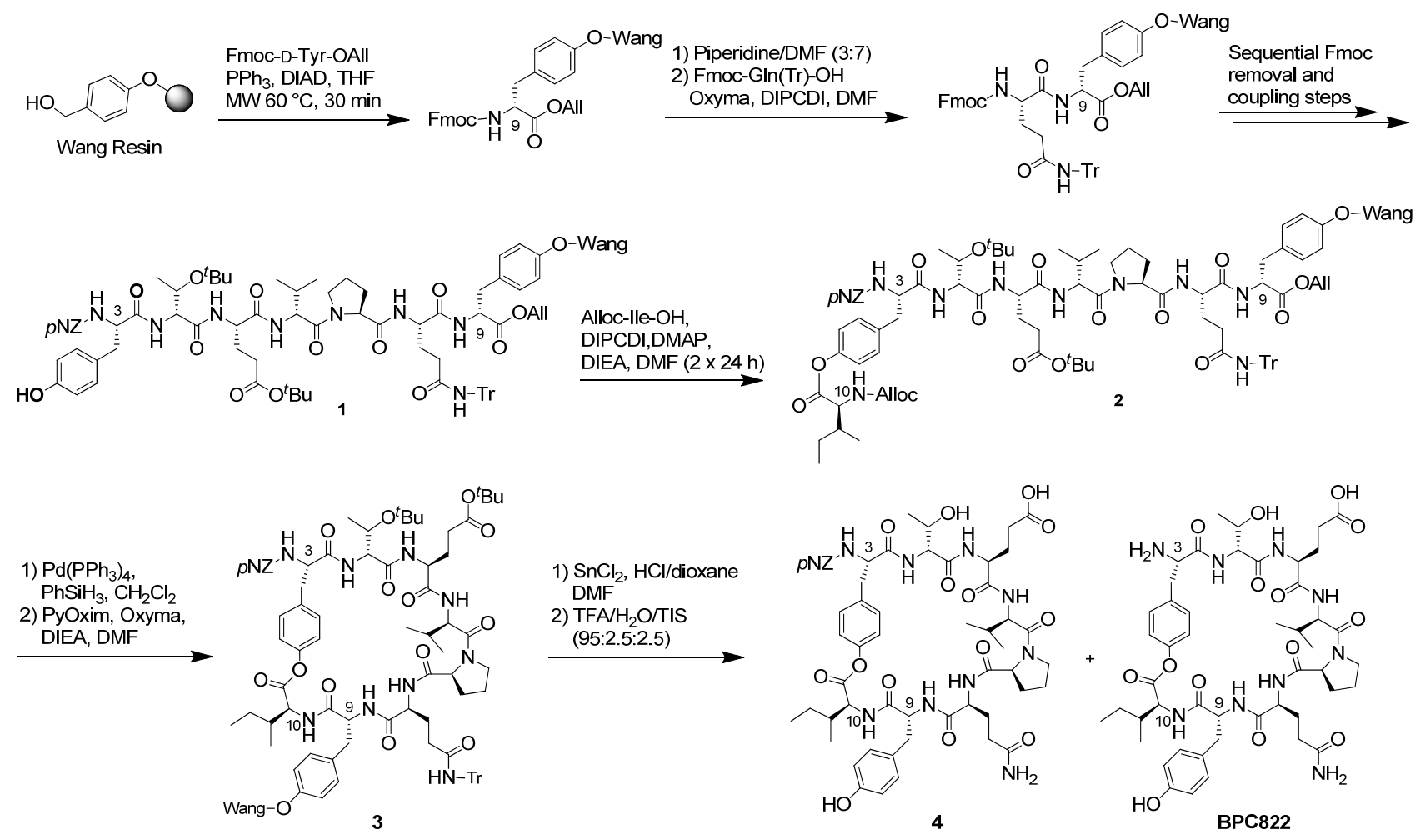

Scheme 2. Synthesis of the cyclic peptidyl resin 3 and attempts of $p$ NZ removal.

Next, the Alloc and allyl protecting groups of resin 2 were simultaneously removed using a catalytic amount of $\mathrm{Pd}\left(\mathrm{PPh}_{3}\right)_{4}$ in presence of $\mathrm{PhSiH}_{3}$ in $\mathrm{CH}_{2} \mathrm{Cl}_{2}$ for $4 \mathrm{~h}$ (Scheme 2). After acidolytic cleavage of an aliquot of the resulting resin $p \mathrm{NZ}-\mathrm{Tyr}^{3}\left(\mathrm{O}-\mathrm{Ile}{ }^{10}-\mathrm{H}\right)-\mathrm{D}-\mathrm{Thr}\left({ }^{t} \mathrm{Bu}\right)-\mathrm{Glu}\left(\mathrm{O}^{t} \mathrm{Bu}\right)-\mathrm{D}-\mathrm{Val}-\mathrm{Pro}-\mathrm{Gln}(\mathrm{Tr})-\mathrm{D}-$ $\operatorname{Tyr}^{9}$ (Wang)-OH, the corresponding peptide was obtained in $72 \%$ HPLC purity. Subsequent on-resin cyclization was assayed using [ethyl cyano(hydroxyimino)acetato- $O^{2}$ ]tri-(1-pyrrolidinyl)-phosphonium hexafluorophosphate (PyOxim), Oxyma and DIEA yielding the cyclic peptidyl resin 3. Treatment of an aliquot of this resin with TFA/ $\mathrm{H}_{2} \mathrm{O} / \mathrm{TIS}$ afforded the cyclic depsipeptide $p$ NZ-Tyr ${ }^{3}(\&)$-D-Thr-Glu-DVal-Pro-Gln-D-Tyr ${ }^{9}-\mathrm{Ile}^{10}-\&$ (4) in 31\% HPLC purity, as shown by ESI-MS where a peak at $\mathrm{m} / z 1195.5$ corresponding to $[\mathrm{M}+\mathrm{Na}]^{+}$was observed (\& symbol indicates the location of the ester linkage ${ }^{39}$ ). The linear peptide precursor was not detected either by HPLC or mass spectrometry. To confirm the 
structure of 4 , the crude reaction mixture resulting from acidolytic cleavage was treated with $\mathrm{CH}_{3} \mathrm{OH} / \mathrm{H}_{2} \mathrm{O} / \mathrm{NH}_{3}(4: 1: 1)$, conditions that are known to hydrolyze ester bonds. ${ }^{40-42}$ HPLC and mass spectrometry analysis of the resulting crude revealed only the presence of the linear peptide resulting from the hydrolysis of the ester bond in $\mathbf{4}, p$ NZ-Tyr ${ }^{3}-\mathrm{D}-\mathrm{Thr}-\mathrm{Glu}-\mathrm{D}-\mathrm{Val}-\mathrm{Pro}-\mathrm{Gln}-\mathrm{D}-\mathrm{Tyr}^{9}-\mathrm{Ile} \mathrm{e}^{10}-\mathrm{OH}$, and of the corresponding methyl ester $p$ NZ-Tyr ${ }^{3}$-D-Thr-Glu-D-Val-Pro-Gln-D-Tyr ${ }^{9}-\mathrm{Ile}^{10}$-OMe. Therefore, this result demonstrated the formation of the cyclic depsipeptide 4.

We then attempted the $p \mathrm{NZ}$ group removal of resin 3 using $\mathrm{SnCl}_{2}(6 \mathrm{M})$ in $\mathrm{HCl} /$ dioxane $(1.6 \mathrm{mM})$ and DMF for $1 \mathrm{~h}$ at room temperature. Unexpectedly, this deprotection resulted to be troublesome and even after repeating this treatment 5 more times, the deprotected cyclic peptide BPC822 was obtained together with an important amount of the $p$ NZ-protected cyclic depsipeptide 4 as shown by mass spectrometry.

Based on these results, we envisaged an alternative route to obtain the cyclic lipodepsipeptide BPC838 (Scheme 3). In this approach, instead of building the macrolactone and then incorporating the tail, we planned the synthesis of the linear peptidyl resin 5a already bearing the acylated dipeptidyl tail. Then, this resin would be esterified at $\mathrm{Tyr}^{3}$ with $\mathrm{Ile}^{10}$ and finally cyclized to render the desired cyclic lipodepsipeptide. 


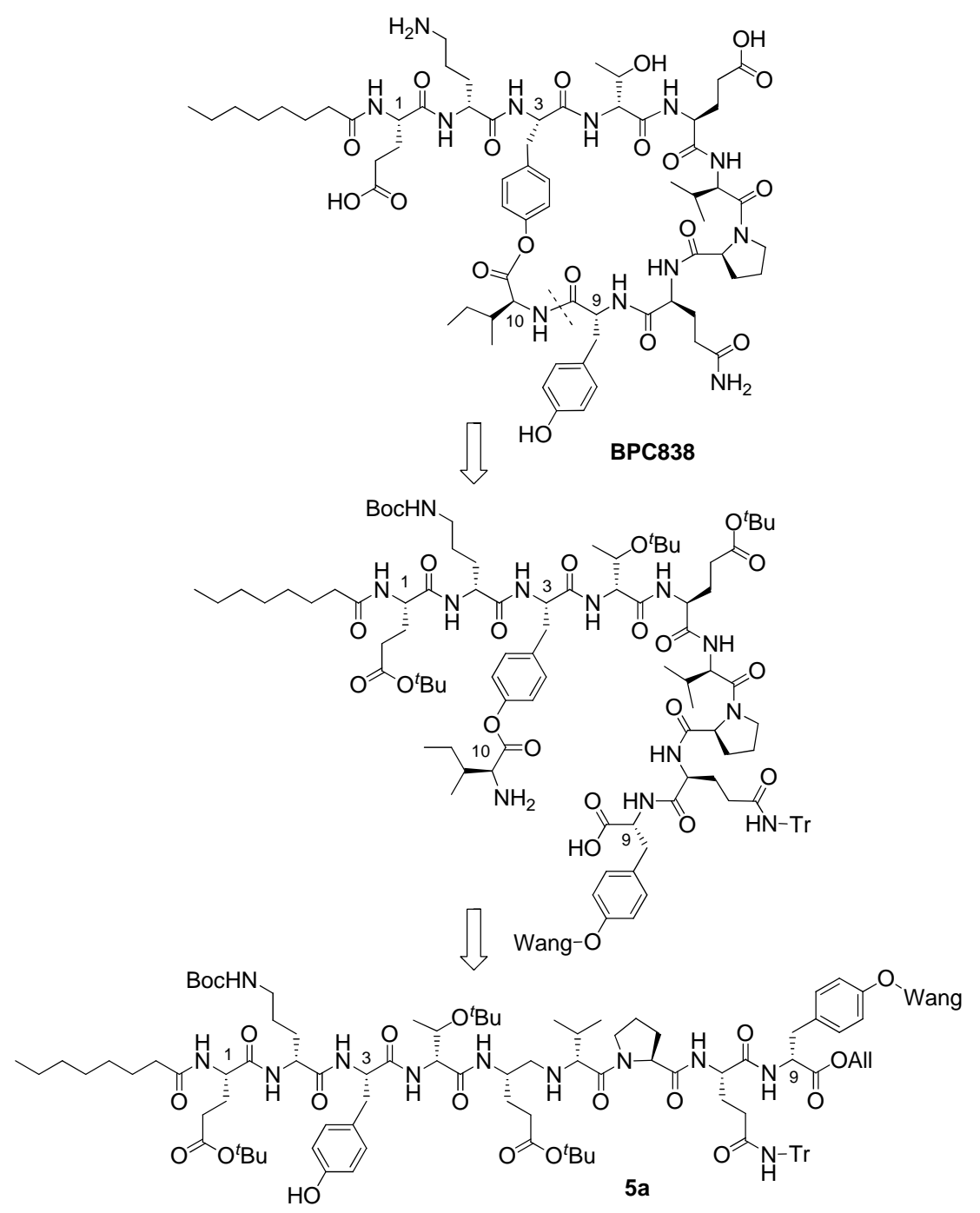

Scheme 3. Alternative retrosynthetic analysis of BPC838.

For this purpose as depicted in Scheme 4, we first synthesized the linear peptidyl resin Fmoc$\mathrm{Glu}^{1}\left(\mathrm{O}^{t} \mathrm{Bu}\right)-\mathrm{D}-\mathrm{Orn}(\mathrm{Boc})-\mathrm{Tyr}^{3}-\mathrm{D}-\mathrm{Thr}\left({ }^{t} \mathrm{Bu}\right)-\mathrm{Glu}\left(\mathrm{O}^{t} \mathrm{Bu}\right)-\mathrm{D}-\mathrm{V}$ al-Pro-Gln$(\mathrm{Tr})-\mathrm{D}-\mathrm{Tyr}^{9}(\mathrm{Wang})-\mathrm{OAll}$ through side-chain anchoring of Fmoc-D-Tyr-OAll to a Wang resin followed by sequential Fmoc removal and coupling steps using the conditions mentioned above for resin $\mathbf{1}$. Orn and $\mathrm{Tyr}^{3}$ were incorporated as Fmoc-Orn(Boc)-OH and Fmoc-Tyr-OH, respectively. An aliquot of the resulting linear 
peptidyl resin 6 was treated with TFA/ $\mathrm{H}_{2} \mathrm{O} / \mathrm{TIS}(95: 2.5: 2.5)$ for $2 \mathrm{~h}$ affording the linear peptide FmocGlu-D-Orn-Tyr-D-Thr-Glu-D-Val-Pro-Gln-D-Tyr-OAll in 74\% HPLC purity.
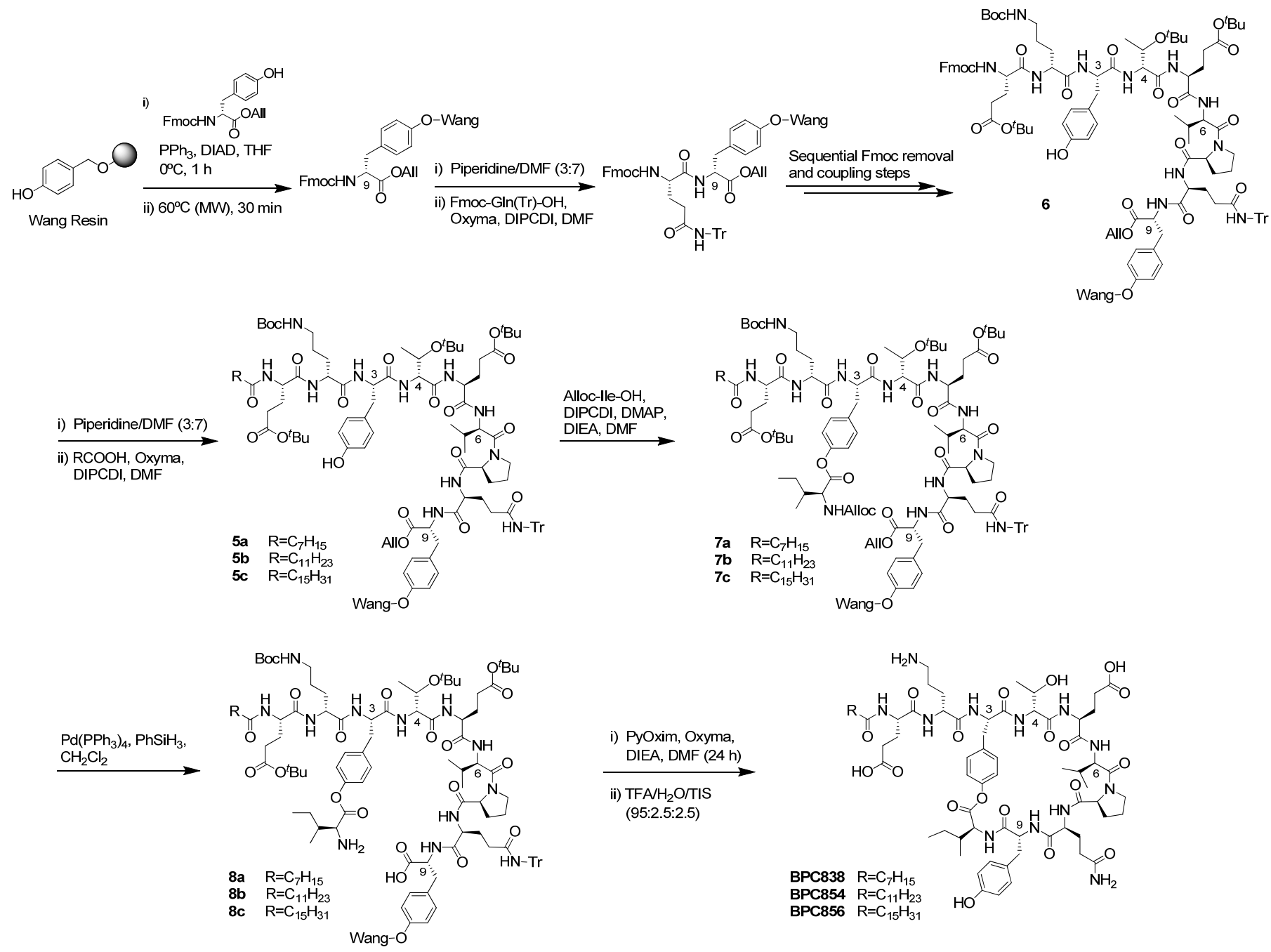

Scheme 4. Structure and general synthetic approach of cyclic lipodepsipeptides BPC838, BPC854 and BPC856.

With peptidyl resin 6 in hand, we proceeded to its acylation with octanoic acid (Scheme 4). Thus, after Fmoc group removal octanoic acid was incorporated using Oxyma/DIPCDI in DMF under stirring at room temperature overnight. Acidolytic treatment of an aliquot of the resulting resin $\mathbf{5 a}$ afforded the 
linear lipopeptide $\mathrm{C}_{7} \mathrm{H}_{15} \mathrm{CO}-\mathrm{Glu}$-D-Orn-Tyr-D-Thr-Glu-D-Val-Pro-Gln-D-Tyr-OAll in 79\% HPLC purity. Next, the esterification of 5a with Alloc-Ile-OH was performed employing DIEA, DIPCDI and DMAP $(2 \times 24 \mathrm{~h})$. An aliquot of the resulting resin $\mathrm{C}_{7} \mathrm{H}_{15} \mathrm{CO}-\mathrm{Glu}^{1}\left(\mathrm{O}^{t} \mathrm{Bu}\right)-\mathrm{D}-\mathrm{Orn}(\mathrm{Boc})-\mathrm{Tyr}^{3}\left(\mathrm{O}-\mathrm{Ile}^{10}-\right.$ Alloc)-D-Thr( $\left.{ }^{t} \mathrm{Bu}\right)-\mathrm{Glu}\left(\mathrm{O}^{t} \mathrm{Bu}\right)-\mathrm{D}-\mathrm{Val}-\mathrm{Pro}-\mathrm{G} \ln (\mathrm{Tr})-\mathrm{D}-\mathrm{Tyr}^{9}($ Wang)-OAll (7a) was acidolytically cleaved yielding the branched lipodepsipeptide $\mathrm{C}_{7} \mathrm{H}_{15} \mathrm{CO}-\mathrm{Glu}$-D-Orn-Tyr(O-Ile-Alloc)-D-Thr-Glu-D-Val-ProGln-D-Tyr-OAll in 81\% HPLC purity.

Selective removal of both Alloc and allyl protecting groups in 7a with $\mathrm{Pd}\left(\mathrm{PPh}_{3}\right)_{3}$ and $\mathrm{PhSiH}_{3}$ in $\mathrm{CH}_{2} \mathrm{Cl}_{2}$ for $4 \mathrm{~h}$ followed by exposure of an aliquot of the resulting resin $8 \mathrm{a}$ to $\mathrm{TFA} / \mathrm{H}_{2} \mathrm{O} / \mathrm{TIS}$ afforded the expected linear peptide in 76\% HPLC purity (Scheme 4). Resin 8a was then treated with Oxyma, PyOxim and DIEA in DMF for $24 \mathrm{~h}$ to prompt its cyclization. Acidolytic cleavage of the resulting resin afforded the desired cyclic lipodepsipeptide $\mathrm{C}_{7} \mathrm{H}_{15} \mathrm{CO}-\mathrm{Glu}-\mathrm{D}-\mathrm{Orn}-\mathrm{Tyr}(\&)$-D-Thr-Glu-D-Val-Pro-Gln-DTyr-Ile-\& (BPC838) in 33\% HPLC purity. Analysis by mass spectrometry of the crude reaction mixture showed the formation of BPC838 as a major product. Interestingly, only traces of the linear peptide resulting from hydrolysis of the macrolactone were observed. Purification by reverse-phase column chromatography (RP-HPLC) yielded BPC838 in $>99 \%$ HPLC purity. The structure of this peptide was verified by $1 \mathrm{D}$ and $2 \mathrm{D}$ NMR experiments. This result demonstrated the suitability of this strategy for the total solid-phase synthesis of dehydroxy fengycin derivatives.

This methodology was then extended to the preparation of cyclic lipodepsipeptides BPC854 and BPC856 bearing a lauric and palmitic acid, respectively (Scheme 4). For this purpose, after Fmoc removal of resin $\mathbf{6}$ acylation of the resulting free amino group was performed using lauric and palmitic acid in presence of DIPCDI and Oxyma. The corresponding lipopeptidyl resins $\mathbf{5 b}$ and $\mathbf{5 c}$ were acydolitically cleaved yielding the expected lipopeptides in 90 and 93\% HPLC purity, respectively. Subsequent esterification of $\mathbf{5 b}$ and $\mathbf{5 c}$ with Alloc-Ile-OH, followed by allyl and Alloc group removal and on-resin cyclization provided, after cleavage with TFA $/ \mathrm{H}_{2} \mathrm{O} / \mathrm{TIS}(95: 2,5: 2,5)$, the cyclic 
lipodepsipeptides $\mathrm{C}_{11} \mathrm{H}_{23} \mathrm{CO}-\mathrm{Glu}-\mathrm{D}-\mathrm{Orn}-\mathrm{Tyr}(\&)$-D-Thr-Glu-D-Val-Pro-Gln-D-Tyr-Ile-\& (BPC854) and $\mathrm{C}_{15} \mathrm{H}_{31}$ CO-Glu-D-Orn-Tyr(\&)-D-Thr-Glu-D-Val-Pro-Gln-D-Tyr-Ile-\& (BPC856) in 49 and 50\% HPLC purity, respectively. Both crude peptides were purified by RP-HPLC and characterized by mass spectrometry yielding BPC854 and BPC856 in good purities (92\% HPLC purity). To confirm the presence of the ester bond, these cyclic lipodepsipeptides were subjected to basic hydrolysis using $\mathrm{CH}_{3} \mathrm{OH} / \mathrm{H}_{2} \mathrm{O} / \mathrm{NH}_{3}(4: 1: 1)$. HPLC and mass spectrometry analysis of the resulting crudes revealed only the presence of the linear peptide resulting from the hydrolysis of the ester bond demonstrating the formation of the cyclic lipodepsipeptide. These peptides were fully characterized by NMR spectroscopy.

Similarly, we prepared analogues of BPC838 differing on the amino acids at positions 4 and 6 (Figure 3). In particular, BPC840 (D-Ser $\left.{ }^{4}, \mathrm{D}^{-\mathrm{Val}^{6}}{ }^{6}, \mathrm{D}-\mathrm{Tyr}^{9}\right)$, BPC842 $\left(\mathrm{D}-\mathrm{Thr}^{4}, \mathrm{D}-\mathrm{Ala}^{6}, \mathrm{D}-\mathrm{Tyr}^{9}\right)$ and BPC844 (D-Ser ${ }^{4}, \mathrm{D}-\mathrm{Ala}^{6}, \mathrm{D}-\mathrm{Tyr}^{9}$ ) were synthesized following the above methodology and were obtained in 35, 26 and 21\% HPLC purity, respectively. ESI-MS analysis showed the presence of the corresponding cyclic lipodepsipeptide as the major product. RP-HPLC purification afforded these peptides in purities $>94 \%$. Their structure was characterized by NMR.

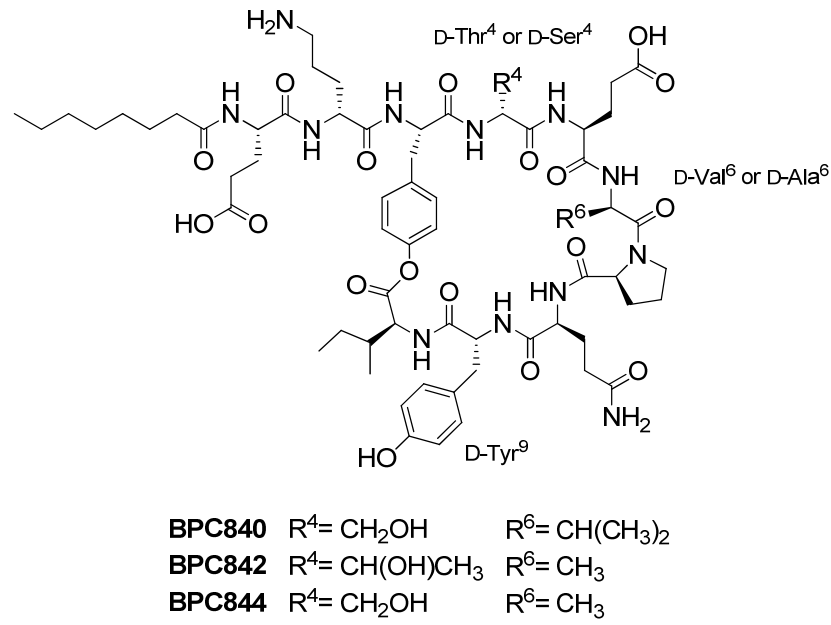

Figure 3. Structure of cyclic lipodepsipeptides BPC840, BPC842 and BPC844. 


\section{Synthesis of Cyclic Lipodepsipeptides Bearing D-Tyr ${ }^{3} / \mathrm{L}^{-T r^{9}}$}

Taken into account the different configuration reported for $\mathrm{Tyr}^{3}$ and $\mathrm{Tyr}^{9}$ for natural fengycins, we decided to prepare cyclic lipodepsipeptides incorporating a $\mathrm{D}-\mathrm{Tyr}^{3}$ and an L-Tyr ${ }^{9}$ (Figure 4). In particular, we attempted the synthesis of BPC846 (D-Tyr ${ }^{3}, \mathrm{D}-\mathrm{Thr}^{4}, \mathrm{D}-\mathrm{Val}^{6}$, octanoyl), BPC848 (D-Tyr ${ }^{3}$, D-Ser ${ }^{4}, \mathrm{D}-\mathrm{Val}^{6}$, octanoyl), BPC850 (D-Tyr ${ }^{3}, \mathrm{D}-\mathrm{Thr}^{4}, \mathrm{D}-\mathrm{Ala}{ }^{6}$, octanoyl $)$, BPC852 (D-Tyr $^{3}, \mathrm{D}-\mathrm{Ser}^{4}, \mathrm{D}-$ Ala ${ }^{6}$, octanoyl), BPC858 (D-Tyr ${ }^{3}, \mathrm{D}^{-T_{h}}{ }^{4}, \mathrm{D}-\mathrm{Val}^{6}$, lauroyl) and BPC860 (D-Tyr ${ }^{3}, \mathrm{D}-\mathrm{Thr}^{4}, \mathrm{D}-\mathrm{Val}^{6}$, palmitoyl).

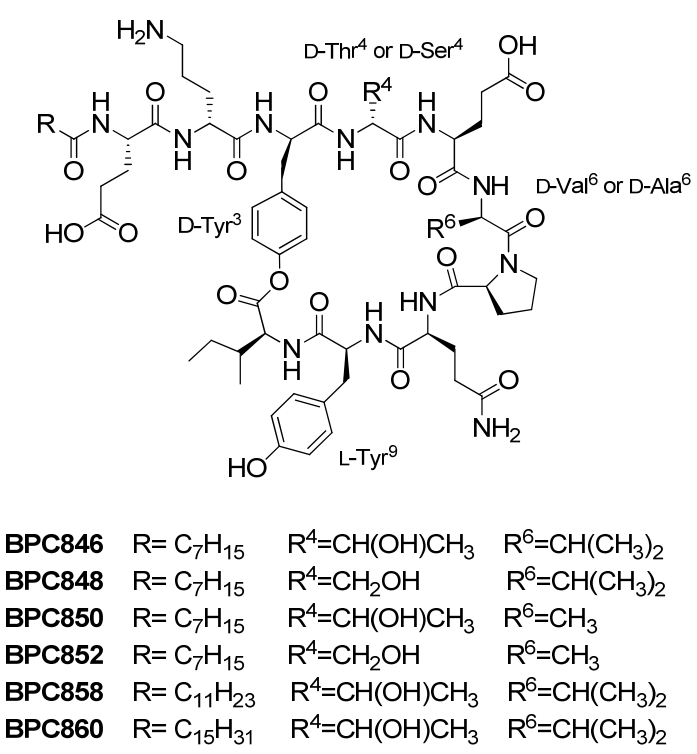

Figure 4. Structure of cyclic lipodepsipeptides BPC846, BPC848, BPC850, BPC852, BPC858 and

\section{BPC860.}

These cyclic lipodepsipeptides were synthesized according to the protocol described in Scheme 4 . After TFA cleavage, mass spectrometry analysis of the crude reaction mixtures showed the presence of the expected peptide along with a substantial amount of a byproduct with a $m / z$ corresponding to $[\mathrm{M}+$ $\left.\mathrm{H}_{2} \mathrm{O}\right]^{+}$. This byproduct could correspond to the linear precursor or to the linear compound resulting from the hydrolysis of the ester bond of the final cyclic depsipeptide. To verify the identity of this 
byproduct, the crude reaction mixtures were subjected to $\mathrm{CH}_{3} \mathrm{OH} / \mathrm{H}_{2} \mathrm{O} / \mathrm{NH}_{3}(4: 1: 1) .{ }^{40-42}$ As depicted in Scheme 5, if the linear precursor is present in the crudes, hydrolysis of the ester bond would lead to the release of the Ile residue (Scheme 5A). However, when the crudes were subjected to hydrolysis only peaks at $\left[\mathrm{M}+\mathrm{H}_{2} \mathrm{O}\right]^{+}$and at $\left[\mathrm{M}+\mathrm{CH}_{3} \mathrm{OH}\right]^{+}$were observed (Scheme 5B). This result demonstrated that the cyclizations were complete and that the cyclic lipodepsipeptides hydrolysed during the cleavage or the HPLC analysis. The crude reaction mixtures from the synthesis of BPC858 and BPC860 were purified by reverse-phase column chromatography and were obtained in 78 and 70\% HPLC purity, respectively.

A
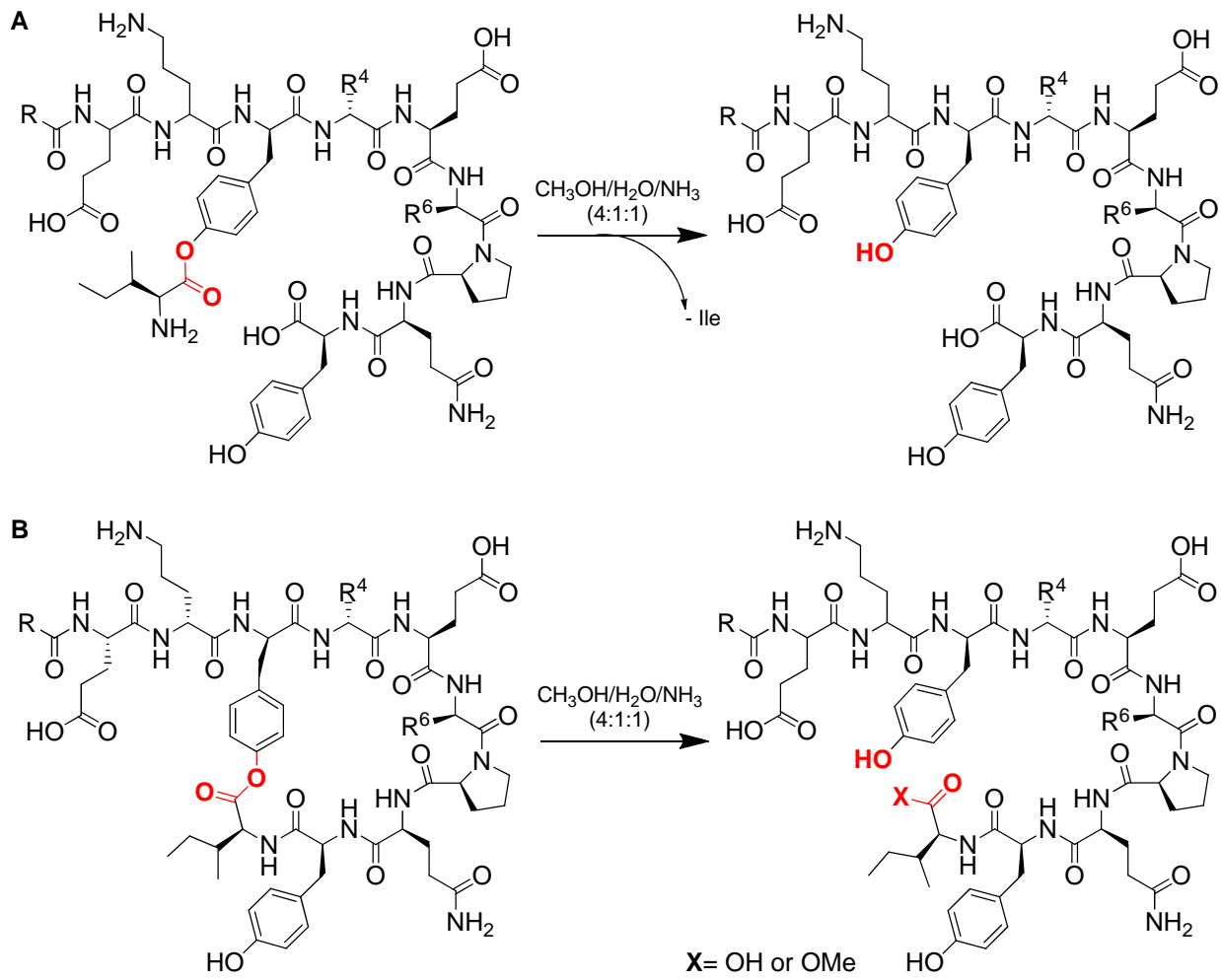

Scheme 5. Hydrolysis reaction of the crude reaction mixtures from the synthesis of the cyclic lipodepsipeptides. A. Hydrolysis of the linear precursors. B. Hydrolysis of the cyclic lipodepsipeptides. 
In our previous studies on the synthesis of the macrolactone of eight amino acids present in fengycins, compounds bearing the same configuration at the Tyr residues than the cyclic lipodepsipeptides depicted in Figure 4 were obtained together with a dimeric product and the linear precursor prior to cyclization. ${ }^{35}$ The results obtained herein point out, on the one hand, that the presence of the fatty acid tail in the linear precursor may favour its cyclization and, on the other hand, that the resulting macrolactone is less stable than the one containing the Tyr residues with an opposite configuration (i.e. BPC846 vs. BPC838). Moreover, these findings further support the recent hypothesis of Honma and co-workers that postulated L-Tyr ${ }^{3} / \mathrm{D}-\mathrm{Tyr}^{9}$ as the correct fengycin configuration. ${ }^{19}$

\section{Conclusion}

In summary, here we describe an efficient solid-phase approach for the total synthesis of dehydroxy derivatives of fengycins $\mathrm{A}, \mathrm{B}$ and $\mathrm{S}$. The synthesis was accomplished using a Fmoc/ ${ }^{t} \mathrm{Bu} / \mathrm{allyl}$ strategy, being the key steps the formation of a phenyl ester and the final macrolactamization. This study revealed the significance of the configuration of the Tyr residues on the stability of the macrolactone and showed that the most stable compounds were those containing an L-Tyr ${ }^{3}$ and a D-Tyr ${ }^{9}$. This study represents the first approach on the total solid-phase synthesis of fengycin derivatives and would allow rapid access to a large variety of analogues. ${ }^{43}$ 


\section{Experimental Section}

\section{General Methods}

Peptide synthesis was performed manually in a polypropylene syringe fitted with a polyethylene porous disc. Solvents and soluble reagents were removed in vacuo. Wang resin was purchased from Fluka. Amino acids derivatives and other chemical reagents were purchased from IrisBiotech, Sigma-Aldrich or Panreac and were used without further purification, unless otherwise noted. Solvents were purchased from Sigma-Aldrich, Sharlau, VidraFoc, SDS or VWR international. All organic solvents were synthesis grade except for $\mathrm{CH}_{3} \mathrm{CN}$ which was multisolvent HPLC grade. Solvents were purified and dried by an activated alumina purification system (mBraun SPS-800) or by conventional distillation techniques. $\mathrm{H}_{2} \mathrm{O}$ was deionised and filtered by a COT Millipore Q-gradient system $(\mathrm{COT}<3 \mathrm{ppb})$ with a resistivity of $18 \mathrm{M} \Omega \cdot \mathrm{cm}^{-1}$.

Microwave-assisted reactions were carried out on a Discover ${ }^{\circledR}$ S-Class CEM Corporation Microwave equipped with an Explorer-Autosampler and controlled with the Synergy ${ }^{\mathrm{TM}}$ software. The equipment was provided with a multispeed magnetic stirring system with adjustable speed and an automated power control system based on temperature feedback through a volume-independent noninvasive infrared sensor control which ranges from 0 to 300 Watts. The work temperature range is from 40 to $300{ }^{\circ} \mathrm{C}$ with a heating rate of $2-6{ }^{\circ} \mathrm{C} /$ second. The reactions were performed in $35 \mathrm{~mL}$ reaction vessels which volume can range from 2 to $25 \mathrm{~mL}$. 
Compounds were analysed under standard analytical high performance liquid chromatography (HPLC) conditions using a Dionex liquid chromatography instrument composed of an UV/Vis Dionex UVD170U detector, a P680 Dionex bomb, an ASI-100 Dionex automatic injector and using the CHROMELEON 6.60 software. The analysis was carried out with a Kromasil $100 \mathrm{C}_{18}(4.6 \mathrm{~mm} \times 40$ $\mathrm{mm}, 3.5 \mu \mathrm{m}$ ) column with a $2-100 \%$ B linear gradient over $7 \mathrm{~min}$ at a flow rate of $1 \mathrm{~mL} / \mathrm{min}$. Solvent A was $0.1 \%$ aq. TFA and solvent $\mathrm{B}$ was $0.1 \% \mathrm{TFA}$ in $\mathrm{CH}_{3} \mathrm{CN}$. Detection was performed at $220 \mathrm{~nm}$. Purity was estimated with the integrated area under peaks. Peptide purifications were carried on a reverse-phase column chromatography CombiFlash Rf200.

MS (ESI) were recorded with an Esquire 6000 ESI Bruker ion Trap LC/MS instrument equipped with an electrospray ion source (Serveis Tècnics de recerca, University of Girona). The instrument was operated in both positive ESI(+) and negative ESI(-) ion modes. Samples $(5 \mu \mathrm{L})$ were introduced into the mass spectrometer ion source directly through a 1200 Series Agilent HPLC autosampler. The mobile phase $\left(80: 20 \mathrm{CH}_{3} \mathrm{CN} / \mathrm{H}_{2} \mathrm{O}\right.$ at a flow rate of $\left.100 \mu \mathrm{L} / \mathrm{min}\right)$ was delivered by an Agilent HPLC pump. Nitrogen was employed as both drying and nebulizing gas.

HRMS analyses were performed under conditions of ESI with a Bruker MicroTOF-QII instrument using a hybrid quadrupole time-of-flight mass spectrometer (Serveis Tècnics de Recerca, University of Girona). Samples were introduced into the mass spectrometer ion source by direct infusion through a syringe pump and were externally calibrated using sodium formate. The instrument was operated in positive $\mathrm{ESI}(+)$ ion mode.

NMR spectra $\left({ }^{1} \mathrm{H}-\mathrm{NMR},{ }^{13} \mathrm{C}-\mathrm{NMR}\right)$ were recorded with a $400 \mathrm{MHz}$ or $300 \mathrm{MHz}$ Bruker Ultrashield Avance spectrometer. Chemical shifts were reported as $\delta$ values ( $\mathrm{ppm}$ ) directly referenced to undeuterated residual solvent signal (i.e. $\mathrm{DMSO}^{-\mathrm{d}_{6}} \delta=2.50 \mathrm{ppm}$ ). The following multiplicity 
abbreviations are used: (d) doublet, (dd) double doublet, (td) triple doublet, (m) multiplet and (br) broad peak. 2D-COSY and 2D-TOCSY experiments were carried out to assign the ${ }^{1} \mathrm{H}-\mathrm{NMR}$ peaks of the cyclic lipodepsipeptides.

IR spectra were recorded with a Bruker Alpha FT-IR spectrometer equipped with a Bruker platinum ATR adaptador and wavenumbers $(v)$ are expressed in $\mathrm{cm}^{-1}$.

The "\&" symbol was used as indicator of the ester chemical bond to facilitate the view of cyclic depsipeptide formulas. On the one-line formula, the "\&" symbol indicates both the location of one end of a chemical bond and the point to which this bond is attached. This symbol has already used in the nomenclature of other cyclic depsipeptides. ${ }^{39}$

Allyl $N^{\alpha}$-(9-fluorenylmethyloxycarbonyl)-D-tyrosinate, allyl $N^{\alpha}$-(9-fluorenylmethyloxycarbonyl)-Ltyrosinate and $N^{\alpha}$-allyloxycarbonyl-L-isoleucine were prepared according to previously reported procedures. $^{35}$

\section{Synthesis of Amino Acids}

$\boldsymbol{N}^{\alpha}$-(p-Nitrobenzyloxycarbonyl)-O-tert-butyl-L-tyrosine. ${ }^{36} \mathrm{NaN}_{3}(164.37 \mathrm{mg}, 2.53 \mathrm{mmol})$ was dissolved in $\mathrm{H}_{2} \mathrm{O}(0.66 \mathrm{~mL})$ and the mixture was added to a solution of $p \mathrm{Nz}-\mathrm{Cl}(468.3 \mathrm{~mL}, 2.11 \mathrm{mmol})$ in 1,4-dioxane $(0.92 \mathrm{~mL})$. The mixture was stirred at room temperature for $2 \mathrm{~h}$ until the formation of $p$ nitrobenzylazidoformate $\left(p \mathrm{Nz}-\mathrm{N}_{3}\right)$. Next, a solution of $\mathrm{H}-\mathrm{L}-\mathrm{Tyr}\left({ }^{t} \mathrm{Bu}\right)-\mathrm{OH}(500 \mathrm{mg}, 2.11 \mathrm{mmols}) \mathrm{in} 1 \%$ $\mathrm{Na}_{2} \mathrm{CO}_{3} / 1$,4-dioxane $(1: 1,2.63 \mathrm{~mL})$ was added dropwise. The resulting white suspension was stirred for $48 \mathrm{~h}$ at room temperature keeping the $\mathrm{pH}$ between 8 and 10 by addition of $10 \% \mathrm{Na}_{2} \mathrm{CO}_{3}$. The progress of the reaction was monitored by TLC. Once the reaction was completed, $\mathrm{H}_{2} \mathrm{O}(30 \mathrm{~mL})$ was added and the resulting suspension was washed with tert-butyl methyl ether $(3 \times 15 \mathrm{~mL})$. The aqueous 
portion was acidified to $\mathrm{pH} 2$ with $3 \mathrm{~N} \mathrm{HCl}$ and a precipitate appeared, which was filtered off and dried to yield $p$ Nz-L-Tyr $\left({ }^{t} \mathrm{Bu}\right)-\mathrm{OH}$ as a white solid $(653.3 \mathrm{mg}, 75 \%$ yield $) .{ }^{1} \mathrm{H}-\mathrm{NMR}\left(400 \mathrm{MHz}, \mathrm{CD}_{3} \mathrm{OD}\right) \delta$ (ppm): $1.31\left(\mathrm{~s}, 9 \mathrm{H},\left(\mathrm{CH}_{3}\right)_{3}\right), 2.87\left(\mathrm{dd}, J=9.6,14.0 \mathrm{~Hz}, 1 \mathrm{H}, \mathrm{CH}_{2}-\beta\right), 3.20(\mathrm{dd}, J=4.6,14.0 \mathrm{~Hz}, 1 \mathrm{H}$, $\left.\mathrm{CH}_{2}-\beta\right), 4.39(\mathrm{dd}, J=4.6,9.6 \mathrm{~Hz}, 1 \mathrm{H}, \mathrm{CH}-\alpha), 5.09\left(\mathrm{~d}, J=13.8 \mathrm{~Hz}, 1 \mathrm{H}, \mathrm{OCH}_{2}\right), 5.19(\mathrm{~d}, J=13.8 \mathrm{~Hz}$, $\left.1 \mathrm{H}, \mathrm{OCH}_{2}\right), 6.88\left(\mathrm{~d}, J=8.6 \mathrm{~Hz}, 2 \mathrm{H}, \mathrm{CH}_{\text {Arom3 }}\right.$-Tyr $), 7.14\left(\mathrm{~d}, J=8.6 \mathrm{~Hz}, 2 \mathrm{H}, \mathrm{CH}_{\text {Arom2 }}\right.$-Tyr $), 7.45(\mathrm{~d}, J=$ $\left.8.8 \mathrm{~Hz}, 2 \mathrm{H}, \mathrm{CH}_{\text {Arom2 }}-p \mathrm{Nz}\right), 8.20\left(\mathrm{~d}, J=8.8 \mathrm{~Hz}, 2 \mathrm{H}, \mathrm{CH}_{\text {Arom } 3}-p \mathrm{Nz}\right) ;{ }^{13} \mathrm{C}\left\{{ }^{1} \mathrm{H}\right\} \mathrm{NMR}\left(400 \mathrm{MHz}, \mathrm{CD}_{3} \mathrm{OD}\right)$ $\delta$ (ppm): $29.2\left(\left(\mathrm{CH}_{3}\right)_{3}\right), 38.3\left(\mathrm{CH}_{2}-\beta\right), 57.3(\mathrm{CH}-\alpha), 65.9\left(\mathrm{OCH}_{2}\right), 79.4\left(\mathrm{C}_{\left.\left(\mathrm{CH}_{3}\right)_{3}\right),}, 124.5,125.1\right.$ $\left(\mathrm{CH}_{\text {Arom3 }}-\mathrm{Ty}, \mathrm{CH}_{\text {Arom3 }}-p \mathrm{Nz}\right), 128.9,130.9\left(\mathrm{CH}_{\text {Arom2 }}-\mathrm{Tyr}, \mathrm{CH}_{\text {Arom2}}-p \mathrm{Nz}\right), 133.9\left(\mathrm{C}_{\text {Arom1 }}-\mathrm{Tyr}\right), 146.1$, $148.8\left(\mathrm{C}_{\text {Arom1- }}-p \mathrm{Nz}, \mathrm{C}_{\text {Arom4 }}-p \mathrm{Nz}\right), 155.3\left(\mathrm{OC}_{\text {Arom4}}-\mathrm{Tyr}\right), 157.8(\mathrm{HN}-\mathrm{C}=\mathrm{O}), 175.7(\mathrm{COOH})$.

$N^{\alpha}$-(p-Nitrobenzyloxycarbonyl)-L-tyrosine. This compound was prepared following the procedure described for Fmoc-L-Tyr-OAll starting from $p \mathrm{Nz}-\mathrm{L}-\mathrm{Tyr}\left({ }^{t} \mathrm{Bu}\right)-\mathrm{OH}(613.3 \mathrm{mg}, 1.47 \mathrm{mmol})$. $p \mathrm{Nz}-\mathrm{L}-\mathrm{Tyr}-$ $\mathrm{OH}$ was obtained quantitatively as a white solid $\left(478.1 \mathrm{mg}, 90 \%\right.$ yield). IR (neat): $3325\left(\mathrm{COO}-\mathrm{H}_{\mathrm{st}}\right)$, $1697\left(\mathrm{C}=\mathrm{O}_{\mathrm{st}}\right), 1608,1512\left(\mathrm{NO}_{2}\right.$ st as $), 1441,1343\left(\mathrm{NO}_{2} \mathrm{st} \operatorname{sim}\right), 1201,1105,1059 \mathrm{~cm}^{-1} ;{ }^{1} \mathrm{H}-\mathrm{NMR}(400$ $\left.\mathrm{MHz}, \mathrm{CD}_{3} \mathrm{OD}\right) \delta(\mathrm{ppm}): 2.82\left(\mathrm{dd}, J=9.6,14.0 \mathrm{~Hz}, 1 \mathrm{H}, \mathrm{CH}_{2}-\beta\right), 3.13\left(\mathrm{dd}, J=4.8,14.0 \mathrm{~Hz}, 1 \mathrm{H}, \mathrm{CH}_{2}-\beta\right)$, $4.38(\mathrm{dd}, J=4.8,9.6 \mathrm{~Hz}, 1 \mathrm{H}, \mathrm{CH}-\alpha), 5.11\left(\mathrm{~d}, J=14.0 \mathrm{~Hz}, 1 \mathrm{H}, \mathrm{OCH}_{2}\right), 5.19(\mathrm{~d}, J=14.0 \mathrm{~Hz}, 1 \mathrm{H}$, $\left.\mathrm{OCH}_{2}\right), 6.70\left(\mathrm{~d}, J=8.4 \mathrm{~Hz}, 2 \mathrm{H}, \mathrm{CH}_{\mathrm{Arom} 3}-\mathrm{Tyr}\right), 7.05\left(\mathrm{~d}, J=8.4 \mathrm{~Hz}, 2 \mathrm{H}, \mathrm{CH}_{\mathrm{Arom} 2}-\mathrm{Tyr}\right), 7.45(\mathrm{~d}, J=8.8$ $\left.\mathrm{Hz}, 2 \mathrm{H}, \mathrm{CH}_{\text {Arom2 } 2}-p \mathrm{Nz}\right), 8.19\left(\mathrm{~d}, J=8.8 \mathrm{~Hz}, 2 \mathrm{H}, \mathrm{CH}_{\text {Arom3 }}-p \mathrm{Nz}\right) ;{ }^{13} \mathrm{C}\left\{{ }^{1} \mathrm{H}\right\} \mathrm{NMR}\left(400 \mathrm{MHz}, \mathrm{CD}_{3} \mathrm{OD}\right) \delta$ (ppm): $37.9\left(\mathrm{CH}_{2}-\beta\right), 57.0(\mathrm{CH}-\alpha), 66.0\left(\mathrm{OCH}_{2}\right), 116.2\left(\mathrm{CH}_{\text {Arom3 }}-\mathrm{Tyr}\right), 124.5\left(\mathrm{CH}_{\text {Arom3 }}-p \mathrm{Nz}\right), 128.8$, 129.2 $\left(\mathrm{CH}_{\text {Arom2 }}-\mathrm{Tyr}, \mathrm{CH}_{\text {Arom2 }}-p \mathrm{Nz}\right), 131.3\left(\mathrm{C}_{\text {Arom } 1}-\mathrm{Tyr}\right), 146.1,148.8\left(\mathrm{C}_{\text {Arom1 }}-p \mathrm{Nz}, \mathrm{C}_{\text {Arom4 } 4}-p \mathrm{Nz}\right), 157.3$, $157.9\left(\mathrm{OC}_{\text {Arom4}}-\mathrm{Tyr}, \mathrm{HN}-\mathrm{C}=\mathrm{O}\right), 175.2(\mathrm{COOH}) ; \mathrm{MS}(\mathrm{ESI}) \mathrm{m} / z: 361.0[\mathrm{M}+\mathrm{H}]^{+}, 383.0[\mathrm{M}+\mathrm{Na}]^{+}$.

$N^{\alpha}$-(9-Fluorenylmethyloxycarbonyl)-D-tyrosine. The commercial amino acid Fmoc-D-Tyr $\left(\mathrm{O}^{t} \mathrm{Bu}\right)-\mathrm{OH}$ $(0.6 \mathrm{~g}, 1.31 \mathrm{mmol})$ was dissolved in TFA $/ \mathrm{CH}_{2} \mathrm{Cl}_{2}(1: 1)$ and stirred at room temperature for $3 \mathrm{~h}$. The solution was concentrated to dryness followed by repeat washings and evaporations with diethyl ether. 
The resulting residue was digested in pentane to yield Fmoc-D-Tyr-OH as a white powder $(0.58 \mathrm{~g}, 97 \%$ yield). IR (neat): $3308\left(\mathrm{OH}_{\mathrm{st}}\right), 1685\left(\mathrm{C}=\mathrm{O}_{\mathrm{st}}\right), 1541,1515\left(\operatorname{arC}-\mathrm{C}_{\mathrm{st}}, \mathrm{NH}_{\delta}\right), 1450\left(\mathrm{CH}_{2 \delta}\right), 1230\left(\operatorname{arC}-\mathrm{O}_{\mathrm{st}}\right)$, $735\left(\mathrm{CH}_{2 \gamma}\right) \mathrm{cm}^{-1} ;{ }^{1} \mathrm{H}-\mathrm{NMR}\left(400 \mathrm{MHz}, \mathrm{DMSO}_{6}\right) \delta(\mathrm{ppm}): 2.74\left(\mathrm{dd}, J=10.8,13.6 \mathrm{~Hz}, 1 \mathrm{H}, \mathrm{CH}_{2}-\beta\right)$, $2.95\left(\mathrm{dd}, J=4.0,13.6 \mathrm{~Hz}, 1 \mathrm{H}, \mathrm{CH}_{2}-\beta\right), 4.05-4.11$ (m, 1H, CH-Fmoc), 4.15-4.21 (m, 3H, OCH $2-\mathrm{Fmoc}_{\text {, }}$ $\mathrm{CH}-\alpha), 6.66$ (d, J = 8.4 Hz, 2H, $\mathrm{CH}_{\text {Arom6-Tyr), }} 7.06$ (d, $J=8.4 \mathrm{~Hz}, 2 \mathrm{H}, \mathrm{CH}_{\text {Arom5-Tyr) }}$, 7.27-7.34 (m, 2H,

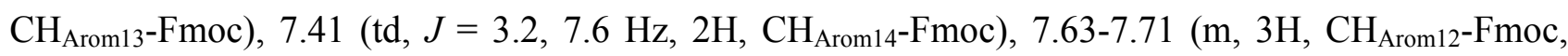
$\mathrm{OH}), 7.88$ (d, $J=7.6 \mathrm{~Hz}, 2 \mathrm{H}, \mathrm{CH}_{\text {Arom } 15}$-Fmoc) 9.22 (br, $\left.1 \mathrm{H}, \mathrm{NH}\right), 12.71$ (br, $\left.1 \mathrm{H}, \mathrm{COOH}\right) ;{ }^{13} \mathrm{C}\left\{{ }^{1} \mathrm{H}\right\}$ NMR (300 MHz, DMSO-d $\left.)_{6}\right) \delta(\mathrm{ppm}): 35.2\left(\mathrm{CH}_{2}-\beta\right), 46.1$ (CH-Fmoc), $55.4(\mathrm{CH}-\alpha), 65.1\left(\mathrm{OCH}_{2^{-}}\right.$ Fmoc), 114.5 ( $\mathrm{CH}_{\text {Arom6 }}$-Tyr $), 119.6,124.8,126.6,127.1,127.5\left(\mathrm{CH}_{\text {Arom12-Fmoc, }} \mathrm{CH}_{\text {Arom13 }}\right.$-Fmoc,

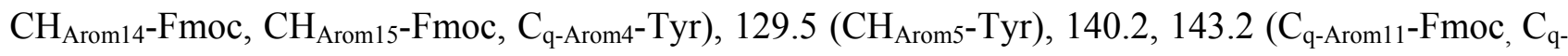
Arom16-Fmoc), 155.4, $155.4\left(\mathrm{OC}_{\mathrm{q}-\mathrm{Arom} 7-}\right.$ Tyr, NH-C=O), $173.0(\mathrm{O}-\mathrm{C}=\mathrm{O})$; MS (ESI) m/z : $404.0[\mathrm{M}+\mathrm{H}]^{+}$, $426.1[\mathrm{M}+\mathrm{Na}]^{+}$.

$N^{\alpha}$-(9-Fluorenylmethyloxycarbonyl)-L-tyrosine. Starting from the commercially available Fmoc-L$\operatorname{Tyr}\left(\mathrm{O}^{t} \mathrm{Bu}\right)-\mathrm{OH}(0.5 \mathrm{~g}, 1.09 \mathrm{mmol})$, this compound was prepared following the procedure described above for Fmoc-D-Tyr-OH. Fmoc-L-Tyr-OH was obtained as a white powder (0.43 g, 96\% yield). IR (neat): $3354\left(\mathrm{OH}_{\mathrm{st}}\right), 1655\left(\mathrm{C}=\mathrm{O}_{\mathrm{st}}\right), 1538,1514\left(\operatorname{arC}-\mathrm{C}_{\mathrm{st}}, \mathrm{NH}_{\delta}\right), 1449\left(\mathrm{CH}_{2 \delta}\right), 1256,1220\left(\operatorname{arC}-\mathrm{O}_{\mathrm{st}}\right), 735$ $\left(\mathrm{CH}_{2 \gamma}\right) \mathrm{cm}^{-1} ;{ }^{1} \mathrm{H}-\mathrm{NMR}\left(400 \mathrm{MHz}, \mathrm{DMSO}-\mathrm{d}_{6}\right) \delta(\mathrm{ppm}): 2.75\left(\mathrm{dd}, J=10.4,13.8 \mathrm{~Hz}, 1 \mathrm{H}, \mathrm{CH}_{2}-\beta\right), 2.95$ (dd, $\left.J=4.4,13.8 \mathrm{~Hz}, 1 \mathrm{H}, \mathrm{CH}_{2}-\beta\right), 4.05-4.11$ (m, 1H, CH-Fmoc), 4.15-4.22 (m, 3H, OCH - Fmoc, CHa), 6.66 (d, $J=8.4 \mathrm{~Hz}, 2 \mathrm{H}, \mathrm{CH}_{\text {Arom6 }}$-Tyr), 7.06 (d, $J=8.4 \mathrm{~Hz}, 2 \mathrm{H}, \mathrm{CH}_{\text {Aroms }}$-Tyr), 7.27-7.34 (m, 2H,

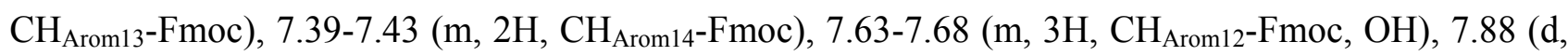
$J=7.2 \mathrm{~Hz}, 2 \mathrm{H}, \mathrm{CH}_{\text {Arom } 15}$-Fmoc), 9.20 (br, 1H, NH), 12.68 (br, $\left.1 \mathrm{H}, \mathrm{COOH}\right) ;{ }^{13} \mathrm{C}\left\{{ }^{1} \mathrm{H}\right\} \mathrm{NMR}(400 \mathrm{MHz}$, DMSO-d $\left.{ }_{6}\right) \delta(\mathrm{ppm}): 36.2\left(\mathrm{CH}_{2}-\beta\right), 47.0(\mathrm{CH}-\mathrm{Fmoc}), 56.3(\mathrm{CH}-\alpha), 66.1\left(\mathrm{OCH}_{2}-\mathrm{Fmoc}\right), 115.4\left(\mathrm{CH}_{\text {Arom6}^{-}}\right.$ Tyr $), 120.5,125.7,127.5,128.1,128.4\left(\mathrm{CH}_{\text {Arom } 12}-\mathrm{Fmoc}, \mathrm{CH}_{\mathrm{Arom} 13}-\mathrm{Fmoc}, \mathrm{CH}_{\mathrm{Arom} 14}-\mathrm{Fmoc}, \mathrm{CH}_{\mathrm{Arom} 15^{-}}\right.$ 
Fmoc, $\mathrm{C}_{\mathrm{q}-\mathrm{Arom} 4}$-Tyr $), 130.48$ ( $\left.\mathrm{CH}_{\text {Arom5-Tyr }}\right), 141.1,144.2$ ( $\mathrm{C}_{\mathrm{q}-\text { Arom11-Fmoc, }} \mathrm{C}_{\mathrm{q}-\mathrm{Arom} 16}$-Fmoc $), 156.3$, $156.4\left(\mathrm{OC}_{\mathrm{q}-\mathrm{Arom7}-\mathrm{Tyr}}\right.$ NH-C=O), $173.9(\mathrm{O}-\mathrm{C}=\mathrm{O})$; MS (ESI) $m / z: 404.0[\mathrm{M}+\mathrm{H}]^{+}, 426.1[\mathrm{M}+\mathrm{Na}]^{+}$.

\section{Solid-Phase Synthesis of Cyclic Lipodepsipeptides}

\section{Synthesis of peptidyl resins 1 and 6, and of the linear lipopeptidyl resins}

Wang resin (450 mg, loading $1.1 \mathrm{mmol} / \mathrm{g}, 100-200 \mathrm{mesh}$, crosslinked with 1\% DVB) was placed in a microwave tube and swollen in dry tetrahydrofuran (THF) for 30 min. A solution of Fmoc-L-Tyr-OAll or Fmoc-D-Tyr-OAll (878.11 mg, $1.98 \mathrm{mmol})$ and triphenylphosphine $\left(\mathrm{PPh}_{3}\right)(519.35 \mathrm{mg}, 1.98 \mathrm{mmol})$ in dry THF $(3 \mathrm{~mL})$ was added to the resin, and the resulting mixture was cooled at $0{ }^{\circ} \mathrm{C}$. A solution of diisopropylazodicarboxylate (DIAD, $390 \mu \mathrm{L}, 1.98 \mathrm{mmol})$ in dry THF $(0.5 \mathrm{~mL})$ was added dropwise and the mixture was stirred $1 \mathrm{~h}$ at $0{ }^{\circ} \mathrm{C}$. The resulting mixture was subjected to microwave irradiaton at $60{ }^{\circ} \mathrm{C}$ for $30 \mathrm{~min}$. Then, the resin was transferred to a polypropylene syringe and washed sequentially with THF $(3 \times 2 \mathrm{~min}), \mathrm{CH}_{2} \mathrm{Cl}_{2}(3 \times 2 \mathrm{~min}), \mathrm{DMF}(3 \times 2 \mathrm{~min}), \mathrm{DMF} / \mathrm{H}_{2} \mathrm{O}(1: 1,3 \times 2 \mathrm{~min}), \mathrm{DMF}(3 \times 2 \mathrm{~min})$, $\mathrm{CH}_{3} \mathrm{OH}(3 \times 2 \mathrm{~min}), \mathrm{CH}_{2} \mathrm{Cl}_{2}(3 \times 2 \mathrm{~min})$ and diethyl ether $(3 \times 2 \mathrm{~min})$, and dried in vacuo. The loading of the resulting resins Fmoc-L-Tyr(Wang)-OAll and Fmoc-D-Tyr(Wang)-OAll was determined using the Fmoc test, being of 0.20 and $0.29 \mathrm{mmol} / \mathrm{g}$, respectively. ${ }^{37}$ The racemization of the anchored amino acid was evaluated using the Marfey's reagent. ${ }^{38}$ Finally, the resin was acetylated with acetic anhydride/pyridine/ $\mathrm{CH}_{2} \mathrm{Cl}_{2}(86: 7: 7,2 \times 30 \mathrm{~min})$. The resin was washed with $\mathrm{CH}_{2} \mathrm{Cl}_{2}(3 \times 2 \mathrm{~min}), \mathrm{DMF}$ $(3 \times 2 \mathrm{~min}), \mathrm{CH}_{3} \mathrm{OH}(3 \times 1 \mathrm{~min}), \mathrm{CH}_{2} \mathrm{Cl}_{2}(3 \times 2 \mathrm{~min})$ and diethyl ether $(3 \times 2 \mathrm{~min})$, and dried in vacuo. ${ }^{44}$

Fmoc-L-Tyr(Wang)-OAll and Fmoc-D-Tyr(Wang)-OAll resins were elongated by the solidphase method following a standard Fmoc chemistry. Fmoc-Gln(Tr)-OH, Fmoc-Pro-OH, Fmoc-D-ValOH, Fmoc-D-Ala-OH, Fmoc-Glu( $\left(\mathrm{O}^{t} \mathrm{Bu}\right)-\mathrm{OH}$, Fmoc-D-Thr $\left({ }^{t} \mathrm{Bu}\right)-\mathrm{OH}$, Fmoc-D-Ser $\left({ }^{t} \mathrm{Bu}\right)-\mathrm{OH}$, Fmoc-Tyr- 
$\mathrm{OH}$, Fmoc-D-Tyr-OH, Fmoc-D-Orn(Boc)-OH and $p \mathrm{NZ}-\mathrm{Tyr}-\mathrm{OH}$ were used as amino acid derivatives. The coupling of the corresponding protected amino acid (4 equiv) was carried out in presence of ethyl 2-cyano-2-(hydroxyimino) acetate (Oxyma) (4 equiv) and N,N'-diisopropylcarbodiimide (DIPCDI) (4 equiv) in DMF for $3 \mathrm{~h}$ under stirring at room temperature. The completion of each coupling was monitored by a Kaiser test ${ }^{45}$ or a chloranil test. ${ }^{46}$ The Fmoc protecting group was removed by treating the resin with piperidine/DMF $(3: 7,1 \times 2+3 \times 10 \mathrm{~min})$. After each coupling and deprotection step, the resin was washed with $\mathrm{DMF}(6 \times 1 \mathrm{~min})$ and $\mathrm{CH}_{2} \mathrm{Cl}_{2}(3 \times 1 \mathrm{~min})$, and air-dried.

To obtain the lipopeptidyl resins, each peptidyl resin was subjected to $N$-terminal Fmoc removal as described above. After washings, the resin was treated with the corresponding fatty acid (3 equiv), Oxyma (3 equiv) and DIPCDI (3 equiv) in DMF under overnight stirring at room temperature. The resin was then washed with $\mathrm{DMF}(6 \times 1 \mathrm{~min})$ and $\mathrm{CH}_{2} \mathrm{Cl}_{2}(3 \times 1 \mathrm{~min})$, and air-dried. Completion of the reaction was checked with the Kaiser test. ${ }^{45}$ An aliquot of each resulting lipopeptidyl resin was treated with trifluoroacetic acid (TFA)/ $\mathrm{H}_{2} \mathrm{O} /$ triisopropylsilane (TIS) $(95: 2.5: 2.5)$ for $2 \mathrm{~h}$ at room temperature. Following TFA evaporation, the resulting crude lipopeptide was precipitated with diethyl ether and then decanted to give a white solid that was taken up in $\mathrm{H}_{2} \mathrm{O} / \mathrm{CH}_{3} \mathrm{CN}(1: 1)$, lyophilized, and analyzed by HPLC and mass spectrometry.

\section{Linear peptidyl resin $p$ NZ-Tyr-D-Thr( $\left.{ }^{t} \mathrm{Bu}\right)-\mathrm{Glu}\left(\mathrm{O}^{t} \mathrm{Bu}\right)-\mathrm{D}-\mathrm{Val}-\mathrm{Pro}-\mathrm{Gln}(\mathrm{Tr})-\mathrm{D}-\mathrm{Tyr}(\mathrm{Wang})-\mathrm{OAll}(1)$.} Starting from Fmoc-D-Tyr(Wang)-OAll, this peptidyl resin was prepared following the above general procedure. Acidolytic cleavage of an aliquot of $\mathbf{1}$ afforded $p$ NZ-Tyr-D-Thr-Glu-D-Val-Pro-Gln-D-TyrOAll in $65 \%$ purity. HPLC $(\lambda=220 \mathrm{~nm}) t_{R}: 7.28 \mathrm{~min}$; MS (ESI) $m / z(+): 1118.5[\mathrm{M}+\mathrm{H}]^{+}, 1140.4$ $[\mathrm{M}+\mathrm{Na}]^{+}, 1156.4[\mathrm{M}+\mathrm{K}]^{+}$. 


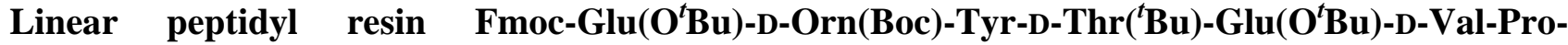

GIn(Tr)-D-Tyr(Wang)-OAll (6). Starting from Fmoc-D-Tyr(Wang)-OAll, this peptidyl resin was prepared following the above general procedure. Acidolytic cleavage of an aliquot of $\mathbf{1}$ afforded FmocGlu-D-Orn-Tyr-D-Thr-Glu-D-Val-Pro-Gln-D-Tyr-OAll in 74\% purity. HPLC $(\lambda=220 \mathrm{~nm}) t_{R}: 7.23 \mathrm{~min}$.

\section{Linear lipopeptidyl resin $\mathrm{C}_{7} \mathrm{H}_{15} \mathrm{CO}-\mathrm{Glu}\left(\mathrm{O}^{t} \mathrm{Bu}\right)-\mathrm{D}-\mathrm{Orn}(\mathrm{Boc})-\mathrm{Tyr}-\mathrm{D}-\mathrm{Thr}\left({ }^{t} \mathrm{Bu}\right)-\mathrm{Glu}\left(\mathrm{O}^{t} \mathrm{Bu}\right)-\mathrm{D}-\mathrm{Val}-$} Pro-GIn(Tr)-D-Tyr(Wang)-OAll (5a). This peptidyl resin was prepared from 6 following the above procedure using octanoic acid as fatty acid. Acidolytic cleavage of an aliquot of $5 \mathbf{a}$ afforded $\mathrm{C}_{7} \mathrm{H}_{15} \mathrm{CO}-$ Glu-D-Orn-Tyr-D-Thr-Glu-D-Val-Pro-Gln-D-Tyr-OAll in 79\% purity. HPLC $(\lambda=220 \mathrm{~nm}) t_{R}=7.09$ $\min$; MS (ESI) $m / z(+): 654.8[\mathrm{M}+2 \mathrm{H}]^{2+}, 1308.7[\mathrm{M}+\mathrm{H}]^{+}, 1330.6[\mathrm{M}+\mathrm{Na}]^{+}$; MS (ESI) $m / z(-): 1306.7$ $[\mathrm{M}-\mathrm{H}]^{-}, 1328.6[\mathrm{M}+\mathrm{Na}-2 \mathrm{H}]^{-}$.

Linear lipopeptidyl resin $\mathrm{C}_{11} \mathrm{H}_{23} \mathrm{CO}-\mathrm{Glu}\left(\mathrm{O}^{t} \mathrm{Bu}\right)-\mathrm{D}-\mathrm{Orn}(\mathrm{Boc})-\mathrm{Tyr}-\mathrm{D}-\mathrm{Thr}\left({ }^{t} \mathrm{Bu}\right)-\mathrm{Glu}\left(\mathrm{O}^{t} \mathrm{Bu}\right)-\mathrm{D}-\mathrm{Val}-$ Pro-GIn(Tr)-D-Tyr(Wang)-OAll (5b). This peptidyl resin was prepared from 6 following the above procedure using lauric acid as fatty acid. Acidolytic cleavage of an aliquot of $\mathbf{5 b}$ afforded $\mathrm{C}_{11} \mathrm{H}_{23} \mathrm{CO}$ Glu-D-Orn-Tyr-D-Thr-Glu-D-Val-Pro-Gln-D-Tyr-OAll in 90\% purity. HPLC $(\lambda=220 \mathrm{~nm}) t_{R}=7.91$ $\min$; MS (ESI) $m / z(+): 683.3[\mathrm{M}+2 \mathrm{H}]^{2+}, 1364.8[\mathrm{M}+\mathrm{H}]^{+}$; MS (ESI) $m / z(-): 1362.7[\mathrm{M}-\mathrm{H}]^{-}, 1384.6$ $[\mathrm{M}+\mathrm{Na}-2 \mathrm{H}]^{-}$.

\section{Linear lipopeptidyl resin $\mathrm{C}_{15} \mathrm{H}_{31} \mathrm{CO}-\mathrm{Glu}\left(\mathrm{O}^{t} \mathrm{Bu}\right)-\mathrm{D}-\mathrm{Orn}(\mathrm{Boc})-\mathrm{Tyr}-\mathrm{D}-\mathrm{Thr}\left({ }^{t} \mathrm{Bu}\right)-\mathrm{Glu}\left(\mathrm{O}^{t} \mathrm{Bu}\right)-\mathrm{D}-\mathrm{Val}-$} Pro-GIn(Tr)-D-Tyr(Wang)-OAll (5c). This peptidyl resin was prepared from 6 following the above procedure using palmitic acid as fatty acid. Acidolytic cleavage of an aliquot of $5 c$ afforded $\mathrm{C}_{15} \mathrm{H}_{31} \mathrm{CO}$ Glu-D-Orn-Tyr-D-Thr-Glu-D-Val-Pro-Gln-D-Tyr-OAll in 93\% purity. HPLC $(\lambda=220 \mathrm{~nm}) t_{R}=8.84$ $\min$; MS (ESI) $m / z(+): 1420.8[\mathrm{M}+\mathrm{H}]^{+}$; MS (ESI) $m / z(-): 1418.7[\mathrm{M}-\mathrm{H}]^{-}, 1440.7[\mathrm{M}+\mathrm{Na}-2 \mathrm{H}]^{-}$. 


\section{Linear lipopeptidyl resin $\mathrm{C}_{7} \mathrm{H}_{15} \mathrm{CO}-\mathrm{Glu}\left(\mathrm{O}^{t} \mathrm{Bu}\right)$-D-Orn(Boc)-Tyr-D-Ser( $\left.{ }^{t} \mathrm{Bu}\right)-\mathrm{Glu}\left(\mathrm{O}^{t} \mathrm{Bu}\right)$-D-Val-Pro-}

GIn(Tr)-D-Tyr(Wang)-OAll. Starting from Fmoc-D-Tyr(Wang)-OAll, this peptidyl resin was prepared following the above general procedure using octanoic acid as fatty acid. Acidolytic cleavage of an

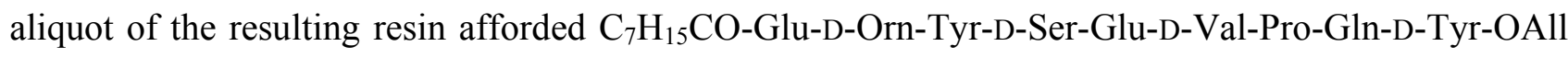
in $67 \%$ purity. $\operatorname{HPLC}(\lambda=220 \mathrm{~nm}) t_{R}=6.97 \mathrm{~min} ; \mathrm{MS}(\mathrm{ESI}) \mathrm{m} / \mathrm{z}(+): 647.8[\mathrm{M}+2 \mathrm{H}]^{2+}, 1294.6[\mathrm{M}+\mathrm{H}]^{+}$; MS (ESI) $m / z(-): 1292.6[\mathrm{M}-\mathrm{H}]^{-}, 1314.5[\mathrm{M}+\mathrm{Na}-2 \mathrm{H}]^{-}$.

\section{Linear lipopeptidyl resin $\mathrm{C}_{7} \mathrm{H}_{15} \mathrm{CO}-\mathrm{Glu}\left(\mathrm{O}^{t} \mathrm{Bu}\right)-\mathrm{D}-\mathrm{Orn}(\mathrm{Boc})-\mathrm{Tyr}-\mathrm{D}-\mathrm{Thr}\left({ }^{t} \mathrm{Bu}\right)-\mathrm{Glu}\left(\mathrm{O}^{t} \mathrm{Bu}\right)-\mathrm{D}-\mathrm{Ala}$ -} Pro-GIn(Tr)-D-Tyr(Wang)-OAll. Starting from Fmoc-D-Tyr(Wang)-OAll, this peptidyl resin was prepared following the above general procedure using octanoic acid as fatty acid. Acidolytic cleavage of an aliquot of the resulting resin afforded $\mathrm{C}_{7} \mathrm{H}_{15} \mathrm{CO}-\mathrm{Glu}-\mathrm{D}-\mathrm{Orn}-\mathrm{Tyr}-\mathrm{D}-\mathrm{Thr}-\mathrm{Glu}-\mathrm{D}-\mathrm{Ala}-\mathrm{Pro}-\mathrm{Gln}-\mathrm{D}-\mathrm{Tyr}-$ OAll in $64 \%$ purity. HPLC $(\lambda=220 \mathrm{~nm}) t_{R}=6.89 \mathrm{~min}$; MS (ESI) $\mathrm{m} / z(+): 641.3[\mathrm{M}+2 \mathrm{H}]^{2+}, 1280.6$ $[\mathrm{M}+\mathrm{H}]^{+} ; \mathrm{MS}(\mathrm{ESI}) \mathrm{m} / z(-): 1278.6[\mathrm{M}-\mathrm{H}]^{-}, 1300.5[\mathrm{M}+\mathrm{Na}-2 \mathrm{H}]^{-}$.

\section{Linear lipopeptidyl resin $\mathrm{C}_{7} \mathrm{H}_{15} \mathrm{CO}-\mathrm{Glu}\left(\mathrm{O}^{t} \mathrm{Bu}\right)$-D-Orn(Boc)-Tyr-D-Ser( $\left.{ }^{t} \mathrm{Bu}\right)-\mathrm{Glu}\left(\mathrm{O}^{t} \mathrm{Bu}\right)$-D-Ala-Pro-} Gln(Tr)-D-Tyr(Wang)-OAll. Starting from Fmoc-D-Tyr(Wang)-OAll, this peptidyl resin was prepared following the above general procedure using octanoic acid as fatty acid. Acidolytic cleavage of an

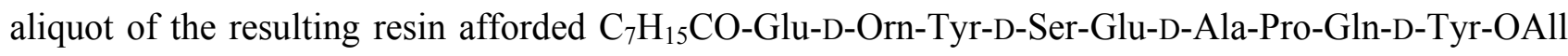
in $71 \%$ purity. $\operatorname{HPLC}(\lambda=220 \mathrm{~nm}) t_{R}=6.84 \mathrm{~min}$; MS (ESI) $\mathrm{m} / \mathrm{z}(+): 633.7[\mathrm{M}+2 \mathrm{H}]^{2+}, 1266.6[\mathrm{M}+\mathrm{H}]^{+}$; MS (ESI) $m / z(-): 1264.6[\mathrm{M}-\mathrm{H}]^{-}, 1286.5[\mathrm{M}+\mathrm{Na}-2 \mathrm{H}]^{-}$.

\section{Linear lipopeptidyl resin $\mathrm{C}_{7} \mathrm{H}_{15} \mathrm{CO}-\mathrm{Glu}\left(\mathrm{O}^{t} \mathrm{Bu}\right)-\mathrm{D}-\mathrm{Orn}(\mathrm{Boc})-\mathrm{D}-\mathrm{Tyr}-\mathrm{D}-\mathrm{Thr}\left({ }^{t} \mathrm{Bu}\right)-\mathrm{Glu}\left(\mathrm{O}^{t} \mathrm{Bu}\right)-\mathrm{D}-\mathrm{Val}-$} Pro-GIn(Tr)-Tyr(Wang)-OAll. Starting from Fmoc-Tyr(Wang)-OAll, this peptidyl resin was prepared following the above general procedure using octanoic acid as fatty acid. Acidolytic cleavage of an aliquot of the resulting resin afforded $\mathrm{C}_{7} \mathrm{H}_{15} \mathrm{CO}-\mathrm{Glu}-\mathrm{D}-\mathrm{Orn}-\mathrm{D}-\mathrm{Tyr}-\mathrm{D}-\mathrm{Thr}-\mathrm{Glu}-\mathrm{D}-\mathrm{Val}-\mathrm{Pro}-\mathrm{Gln}-\mathrm{Tyr}-$ 
OAll in $69 \%$ purity. HPLC $(\lambda=220 \mathrm{~nm}) t_{R}=7.00 \mathrm{~min}$; MS (ESI) $\mathrm{m} / z(+): 1308.6[\mathrm{M}+\mathrm{H}]^{+}$; MS (ESI) $m / z(-): 1306.6[\mathrm{M}-\mathrm{H}]^{-}$.

\section{Linear lipopeptidyl resin $\mathrm{C}_{7} \mathrm{H}_{15} \mathrm{CO}-\mathrm{Glu}\left(\mathrm{O}^{t} \mathrm{Bu}\right)$-D-Orn(Boc)-D-Tyr-D-Ser( $\left.{ }^{t} \mathrm{Bu}\right)-\mathrm{Glu}\left(\mathrm{O}^{t} \mathrm{Bu}\right)-\mathrm{D}-\mathrm{Val}-$} Pro-GIn(Tr)-Tyr(Wang)-OAll. Starting from Fmoc-Tyr(Wang)-OAll, this peptidyl resin was prepared following the above general procedure using octanoic acid as fatty acid. Acidolytic cleavage of an aliquot of the resulting resin afforded $\mathrm{C}_{7} \mathrm{H}_{15} \mathrm{CO}-\mathrm{Glu}$-D-Orn-D-Tyr-D-Ser-Glu-D-Val-Pro-Gln-TyrOAll in $67 \%$ purity. HPLC $(\lambda=220 \mathrm{~nm}) t_{R}=6.97 \mathrm{~min}$; MS (ESI) $\mathrm{m} / z(+): 647.7[\mathrm{M}+2 \mathrm{H}]^{2+}, 1294.6$ $[\mathrm{M}+\mathrm{H}]^{+}, 1316.6[\mathrm{M}+\mathrm{Na}]^{+}$; MS (ESI) $m / z(-): 1292.6[\mathrm{M}-\mathrm{H}]^{-}, 1314.5[\mathrm{M}+\mathrm{Na}-2 \mathrm{H}]^{-}$.

\section{Linear lipopeptidyl resin $\mathrm{C}_{7} \mathrm{H}_{15} \mathrm{CO}-\mathrm{Glu}\left(\mathrm{O}^{t} \mathrm{Bu}\right)-\mathrm{D}-\mathrm{Orn}(\mathrm{Boc})-\mathrm{D}-\mathrm{Tyr}-\mathrm{D}-\mathrm{Thr}\left({ }^{t} \mathrm{Bu}\right)-\mathrm{Glu}\left(\mathrm{O}^{t} \mathrm{Bu}\right)$-D-Ala-} Pro-GIn(Tr)-Tyr(Wang)-OAll. Starting from Fmoc-Tyr(Wang)-OAll, this peptidyl resin was prepared following the above general procedure using octanoic acid as fatty acid. Acidolytic cleavage of an aliquot of the resulting resin afforded $\mathrm{C}_{7} \mathrm{H}_{15} \mathrm{CO}-$ Glu-D-Orn-D-Tyr-D-Thr-Glu-D-Ala-Pro-Gln-TyrOAll in $68 \%$ purity. HPLC $(\lambda=220 \mathrm{~nm}) t_{R}=6.89 \mathrm{~min}$; MS (ESI) $\mathrm{m} / z(+): 1280.6[\mathrm{M}+\mathrm{H}]^{+}, 1302.6$ $[\mathrm{M}+\mathrm{Na}]^{+}$; MS (ESI) $m / z(-): 1278.6[\mathrm{M}-\mathrm{H}]^{-}, 1300.5[\mathrm{M}+\mathrm{Na}-2 \mathrm{H}]^{-}$.

Linear lipopeptidyl resin $\mathrm{C}_{7} \mathrm{H}_{15} \mathrm{CO}-\mathrm{Glu}\left(\mathrm{O}^{t} \mathrm{Bu}\right)$-D-Orn(Boc)-D-Tyr-D-Ser( $\left.{ }^{t} \mathrm{Bu}\right)-\mathrm{Glu}\left(\mathrm{O}^{t} \mathrm{Bu}\right)-\mathrm{D}-\mathrm{Ala}$ Pro-GIn(Tr)-Tyr(Wang)-OAll. Starting from Fmoc-Tyr(Wang)-OAll, this peptidyl resin was prepared following the above general procedure using octanoic acid as fatty acid. Acidolytic cleavage of an aliquot of the resulting resin afforded $\mathrm{C}_{7} \mathrm{H}_{15} \mathrm{CO}-\mathrm{Glu}-\mathrm{D}-\mathrm{Orn}-\mathrm{D}-\mathrm{Tyr}-\mathrm{D}-\mathrm{Ser}-\mathrm{Glu}-\mathrm{D}-\mathrm{Ala}-\mathrm{Pro}-\mathrm{Gln}-\mathrm{Tyr}-$ OAll in $67 \%$ purity. HPLC $(\lambda=220 \mathrm{~nm}) t_{R}=6.85 \mathrm{~min}$; MS (ESI) $\mathrm{m} / z(+): 1266.6[\mathrm{M}+\mathrm{H}]^{+}, 1288.6$ $[\mathrm{M}+\mathrm{Na}]^{+} ; \mathrm{MS}(\mathrm{ESI}) \mathrm{m} / z(-): 1264.6[\mathrm{M}-\mathrm{H}]^{-}, 1286.5[\mathrm{M}+\mathrm{Na}-2 \mathrm{H}]^{-}$. 


\section{Linear lipopeptidyl resin $\mathrm{C}_{11} \mathrm{H}_{23} \mathrm{CO}-\mathrm{Glu}\left(\mathrm{O}^{t} \mathrm{Bu}\right)$-D-Orn(Boc)-D-Tyr-D-Thr( $\left.{ }^{t} \mathrm{Bu}\right)-\mathrm{Glu}\left(\mathrm{O}^{t} \mathrm{Bu}\right)-\mathrm{D}-\mathrm{Val}$ -}

Pro-GIn(Tr)-Tyr(Wang)-OAll. Starting from Fmoc-Tyr(Wang)-OAll, this peptidyl resin was prepared following the above general procedure using lauric acid as fatty acid. Acidolytic cleavage of an aliquot of the resulting resin afforded $\mathrm{C}_{11} \mathrm{H}_{23} \mathrm{CO}-\mathrm{Glu}$-D-Orn-D-Tyr-D-Thr-Glu-D-Val-Pro-Gln-TyrOAll in $66 \%$ purity. HPLC $(\lambda=220 \mathrm{~nm}) t_{R}=8.14 \mathrm{~min} ; \mathrm{MS}(\mathrm{ESI}) \mathrm{m} / z(+): 1364.7[\mathrm{M}+\mathrm{H}]^{+}, 1386.7$ $[\mathrm{M}+\mathrm{Na}]^{+}$; MS (ESI) $m / z(-): 1362.7[\mathrm{M}-\mathrm{H}]^{-}, 1384.6[\mathrm{M}+\mathrm{Na}-2 \mathrm{H}]^{-}$.

\section{Linear lipopeptidyl resin $\mathrm{C}_{15} \mathrm{H}_{31} \mathrm{CO}-\mathrm{Glu}\left(\mathrm{O}^{t} \mathrm{Bu}\right)-\mathrm{D}-\mathrm{Orn}(\mathrm{Boc})-\mathrm{D}-\mathrm{Tyr}-\mathrm{D}-\mathrm{Thr}\left({ }^{t} \mathrm{Bu}\right)-\mathrm{Glu}\left(\mathrm{O}^{t} \mathrm{Bu}\right)-\mathrm{D}-\mathrm{Val}-$} Pro-GIn(Tr)-Tyr(Wang)-OAll. Starting from Fmoc-Tyr(Wang)-OAll, this peptidyl resin was prepared following the above general procedure using palmitic acid as fatty acid. Acidolytic cleavage of an aliquot of the resulting resin afforded $\mathrm{C}_{15} \mathrm{H}_{31} \mathrm{CO}-\mathrm{Glu}-\mathrm{D}-\mathrm{Orn}-\mathrm{D}-\mathrm{Tyr}-\mathrm{D}-\mathrm{Thr}$-Glu-D-Val-Pro-GlnTyr-OAll in 79\% purity. HPLC $(\lambda=220 \mathrm{~nm}) t_{R}=9.11 \mathrm{~min}$; MS (ESI) $m / z(+): 710.8[\mathrm{M}+2 \mathrm{H}]^{2+}, 1420.8$ $[\mathrm{M}+\mathrm{H}]^{+}, 1442.8[\mathrm{M}+\mathrm{Na}]^{+}$; MS (ESI) $m / z(-): 708.8[\mathrm{M}-2 \mathrm{H}]^{2-}, 1418.7[\mathrm{M}-\mathrm{H}]^{-}, 1440.7[\mathrm{M}+\mathrm{Na}-2 \mathrm{H}]^{-}$.

\section{Synthesis of linear depsipeptidyl resins containing a phenyl ester}

Each peptidyl resin was treated with Alloc-L-Ile-OH (7 equiv), DIEA (1.4 equiv), DIPCDI (7 equiv) and DMAP (0.7 equiv) in DMF for $24 \mathrm{~h}$ at room temperature. This treatment was repeated twice. After each treatment, the resin was washed with $\mathrm{DMF}(6 \times 1 \mathrm{~min}), \mathrm{CH}_{2} \mathrm{Cl}_{2}(3 \times 1 \mathrm{~min})$ and air-dried. An aliquot of the each resulting resin was treated with TFA/ $\mathrm{H}_{2} \mathrm{O} / \mathrm{TIS}(95: 2.5: 2.5)$ for $2 \mathrm{~h}$ at room temperature. Following TFA evaporation and diethyl ether extraction, the crude peptide was dissolved in $\mathrm{H}_{2} \mathrm{O} / \mathrm{CH}_{3} \mathrm{CN}$, lyophilized, analyzed by HPLC and characterized by mass spectrometry. 
Linear depsipeptidyl resin $p$ NZ-Tyr(O-Ile-Alloc)-D-Thr( $\left.{ }^{t} \mathrm{Bu}\right)-\mathrm{Glu}\left(\mathrm{O}^{t} \mathrm{Bu}\right)-\mathrm{D}-\mathrm{Val}-\mathrm{Pro}-\mathrm{G} \ln (\mathrm{Tr})-\mathrm{D}-$ Tyr(Wang)-OAll (2). Starting from 1, this depsipeptidyl resin was prepared according to the general procedure described above. Acidolytic cleavage of an aliquot of 2 afforded $p$ NZ-Tyr(O-Ile-Alloc)-DThr-Glu-D-Val-Pro-Gln-D-Tyr-OAll in $87 \%$ purity. HPLC $(\lambda=220 \mathrm{~nm}) t_{R}=8.21 \mathrm{~min}$; MS (ESI) $m / z(+): 1315.6[\mathrm{M}+\mathrm{H}]^{+}, 1337.6[\mathrm{M}+\mathrm{Na}]^{+}, 1353.5[\mathrm{M}+\mathrm{K}]^{+}$.

Linear lipodepsipeptidyl resin $\mathrm{C}_{7} \mathrm{H}_{15} \mathrm{CO}-\mathrm{Glu}\left(\mathrm{O}^{t} \mathrm{Bu}\right)-\mathrm{D}-\mathrm{Orn}(\mathrm{Boc})-\mathrm{Tyr}(\mathrm{O}-\mathrm{Ile}-\mathrm{Alloc})-\mathrm{D}-\mathrm{Thr}\left({ }^{t} \mathrm{Bu}\right)-$ Glu(O ${ }^{t}$ Bu)-D-Val-Pro-GIn(Tr)-D-Tyr(Wang)-OAll (7a). Starting from 5a, this depsipeptidyl resin was prepared according to the general procedure described above. Acidolytic cleavage of an aliquot of 7a afforded $\mathrm{C}_{7} \mathrm{H}_{15} \mathrm{CO}-\mathrm{Glu}-\mathrm{D}-\mathrm{Orn}-\mathrm{Tyr}(\mathrm{O}-\mathrm{Ile}-\mathrm{Alloc})-\mathrm{D}-\mathrm{Thr}-\mathrm{Glu}-\mathrm{D}-\mathrm{Val}-\mathrm{Pro}-\mathrm{G} \ln -\mathrm{D}-\mathrm{Tyr}-\mathrm{OAll}$ in $81 \%$ purity. HPLC $(\lambda=220 \mathrm{~nm}) t_{R}=7.82 \mathrm{~min} ; \mathrm{MS}(\mathrm{ESI}) \mathrm{m} / z(+): 753.3[\mathrm{M}+2 \mathrm{H}]^{2+}, 1505.7[\mathrm{M}+\mathrm{H}]^{+} ; \mathrm{MS}$ (ESI) $m / z(-): 1503.7[\mathrm{M}-\mathrm{H}]^{-}, 1525.6[\mathrm{M}+\mathrm{Na}-2 \mathrm{H}]^{-}$.

Linear lipodepsipeptidyl resin $\mathrm{C}_{11} \mathrm{H}_{23} \mathrm{CO}-\mathrm{Glu}\left(\mathrm{O}^{t} \mathrm{Bu}\right)$-D-Orn(Boc)-Tyr(O-Ile-Alloc)-D-Thr( $\left.{ }^{t} \mathrm{Bu}\right)$ Glu(Ot Bu)-D-Val-Pro-GIn(Tr)-D-Tyr(Wang)-OAll (7b). Starting from 5b, this depsipeptidyl resin was prepared according to the general procedure described above. Acidolytic cleavage of an aliquot of 7b afforded $\mathrm{C}_{11} \mathrm{H}_{23} \mathrm{CO}-\mathrm{Glu}-\mathrm{D}-\mathrm{Orn}-\mathrm{Tyr}(\mathrm{O}-\mathrm{Ile}-\mathrm{Alloc})-\mathrm{D}-\mathrm{Thr}-\mathrm{Glu}-\mathrm{D}-\mathrm{Val}-\mathrm{Pro}-\mathrm{Gln}$-D-Tyr-OAll in $88 \%$ purity. HPLC $(\lambda=220 \mathrm{~nm}) t_{R}=8.72 \mathrm{~min} ; \mathrm{MS}(\mathrm{ESI}) \mathrm{m} / z(+): 781.4[\mathrm{M}+2 \mathrm{H}]^{2+}, 1561.9[\mathrm{M}+\mathrm{H}]^{+}$; MS (ESI) $m / z(-): 1559.8[\mathrm{M}-\mathrm{H}]^{-}, 1581.7[\mathrm{M}+\mathrm{Na}-2 \mathrm{H}]^{-}$.

Linear lipodepsipeptidyl resin $\mathrm{C}_{15} \mathrm{H}_{31} \mathrm{CO}-\mathrm{Glu}\left(\mathrm{O}^{t} \mathrm{Bu}\right)-\mathrm{D}-\mathrm{Orn}(\mathrm{Boc})-\mathrm{Tyr}(\mathrm{O}-\mathrm{Ile}-\mathrm{Alloc})-\mathrm{D}-\mathrm{Thr}\left({ }^{t} \mathrm{Bu}\right)$ Glu(O'Bu)-D-Val-Pro-GIn(Tr)-D-Tyr(Wang)-OAll (7c). Starting from 5c, this depsipeptidyl resin was prepared according to the general procedure described above. Acidolytic cleavage of an aliquot of 7c afforded $\mathrm{C}_{15} \mathrm{H}_{31} \mathrm{CO}-\mathrm{Glu}-\mathrm{D}-\mathrm{Orn}-\mathrm{Ty}(\mathrm{O}-\mathrm{Il}-\mathrm{Alloc})-\mathrm{D}-\mathrm{Thr}-\mathrm{Glu}-\mathrm{D}-\mathrm{Val}-\mathrm{Pro}-\mathrm{Gln}-\mathrm{D}-\mathrm{Tyr}-\mathrm{OAll}$ in $88 \%$ 
purity. HPLC $(\lambda=220 \mathrm{~nm}) t_{R}=9.74 \mathrm{~min} ; \mathrm{MS}(\mathrm{ESI}) \mathrm{m} / z(+): 809.5[\mathrm{M}+2 \mathrm{H}]^{2+}, 1617.9[\mathrm{M}+\mathrm{H}]^{+}$; MS (ESI) $m / z(-): 1615.8[\mathrm{M}-\mathrm{H}]^{-}, 1637.8[\mathrm{M}+\mathrm{Na}-2 \mathrm{H}]^{-}$.

\section{Linear lipodepsipeptidyl resin $\mathrm{C}_{7} \mathrm{H}_{15} \mathrm{CO}-\mathrm{Glu}\left(\mathrm{O}^{t} \mathrm{Bu}\right)-\mathrm{D}-\mathrm{Orn}(\mathrm{Boc})-\mathrm{Tyr}(\mathrm{O}-\mathrm{Ile}-\mathrm{Alloc})-\mathrm{D}-\mathrm{Ser}\left({ }^{t} \mathrm{Bu}\right)-$} Glu(O'Bu)-D-Val-Pro-GIn(Tr)-D-Tyr(Wang)-OAll. Starting from $\mathrm{C}_{7} \mathrm{H}_{15} \mathrm{CO}-\mathrm{Glu}\left(\mathrm{O}^{t} \mathrm{Bu}\right)$-D-Orn(Boc)Tyr-D-Ser $\left({ }^{t} \mathrm{Bu}\right)-\mathrm{Glu}\left(\mathrm{O}^{t} \mathrm{Bu}\right)-\mathrm{D}-\mathrm{Val}-\mathrm{Pro}-\mathrm{G} \ln (\mathrm{Tr})-\mathrm{D}-\mathrm{Tyr}(\mathrm{Wang})-\mathrm{OAll}$, this depsipeptidyl resin was prepared according to the general procedure described above. Acidolytic cleavage of an aliquot of the resulting resin afforded $\mathrm{C}_{7} \mathrm{H}_{15} \mathrm{CO}-\mathrm{Glu}-\mathrm{D}-\mathrm{Orn}-\mathrm{Tyr}(\mathrm{O}-\mathrm{Ile}-\mathrm{Alloc})-\mathrm{D}-\mathrm{Ser}-\mathrm{Glu}-\mathrm{D}-\mathrm{Val}-\mathrm{Pro}-\mathrm{Gln}-\mathrm{D}-\mathrm{Tyr}-\mathrm{OAll}$ in $81 \%$ purity. $\operatorname{HPLC}(\lambda=220 \mathrm{~nm}) t_{R}=7.72 \mathrm{~min} ; \mathrm{MS}(\mathrm{ESI}) \mathrm{m} / \mathrm{z}(+): 746.3[\mathrm{M}+2 \mathrm{H}]^{2+}, 1491.7[\mathrm{M}+\mathrm{H}]^{+}$; MS (ESI) $m / z(-): 1489.7[\mathrm{M}-\mathrm{H}]^{-}, 1512.6[\mathrm{M}+\mathrm{Na}-2 \mathrm{H}]^{-}$.

\section{Linear lipodepsipeptidyl resin $\mathrm{C}_{7} \mathrm{H}_{15} \mathrm{CO}-\mathrm{Glu}\left(\mathrm{O}^{t} \mathrm{Bu}\right)-\mathrm{D}-\mathrm{Orn}(\mathrm{Boc})-\mathrm{Tyr}(\mathrm{O}-\mathrm{Ile}-\mathrm{Alloc})-\mathrm{D}-\mathrm{Thr}\left({ }^{t} \mathrm{Bu}\right)$ -} Glu(O'Bu)-D-Ala-Pro-GIn(Tr)-D-Tyr(Wang)-OAll. Starting from $\mathrm{C}_{7} \mathrm{H}_{15} \mathrm{CO}-\mathrm{Glu}\left(\mathrm{O}^{t} \mathrm{Bu}\right)$-D-Orn(Boc)Tyr-D-Thr $\left({ }^{t} \mathrm{Bu}\right)-\mathrm{Glu}\left(\mathrm{O}^{t} \mathrm{Bu}\right)-\mathrm{D}-\mathrm{Ala}-\mathrm{Pro}-\mathrm{G} \ln (\mathrm{Tr})-\mathrm{D}-\mathrm{Tyr}($ Wang)-OAll, this depsipeptidyl resin was prepared according to the general procedure described above. Acidolytic cleavage of an aliquot of the resulting resin afforded $\mathrm{C}_{7} \mathrm{H}_{15} \mathrm{CO}-\mathrm{Glu}-\mathrm{D}-\mathrm{Orn}-\mathrm{Tyr}(\mathrm{O}-\mathrm{Ile}-\mathrm{Alloc})-\mathrm{D}-\mathrm{Thr}$-Glu-D-Ala-Pro-Gln-D-Tyr-OAll in $77 \%$ purity. $\operatorname{HPLC}(\lambda=220 \mathrm{~nm}) t_{R}=7.68 \mathrm{~min}$; MS (ESI) $m / z(+): 739.8[\mathrm{M}+2 \mathrm{H}]^{2+}, 1477.7[\mathrm{M}+\mathrm{H}]^{+}$; MS (ESI) $m / z(-): 1475.6[\mathrm{M}-\mathrm{H}]^{-}, 1497.6[\mathrm{M}+\mathrm{Na}-2 \mathrm{H}]^{-}$.

\section{Linear lipodepsipeptidyl resin $\mathrm{C}_{7} \mathrm{H}_{15} \mathrm{CO}-\mathrm{Glu}\left(\mathrm{O}^{t} \mathrm{Bu}\right)-\mathrm{D}-\mathrm{Orn}(\mathrm{Boc})-\mathrm{Tyr}\left(\mathrm{O}-\mathrm{Ile}\right.$-Alloc)-D-Ser( $\left.{ }^{t} \mathrm{Bu}\right)-$} Glu(O'Bu)-D-Ala-Pro-Gln(Tr)-D-Tyr(Wang)-OAll. Starting from $\mathrm{C}_{7} \mathrm{H}_{15} \mathrm{CO}-\mathrm{Glu}\left(\mathrm{O}^{t} \mathrm{Bu}\right)$-D-Orn(Boc)Tyr-D-Ser $\left({ }^{t} \mathrm{Bu}\right)-\mathrm{Glu}\left(\mathrm{O}^{t} \mathrm{Bu}\right)-\mathrm{D}-\mathrm{Ala}-\mathrm{Pro}-\mathrm{G} \ln (\mathrm{Tr})-\mathrm{D}-\mathrm{Tyr}(\mathrm{Wang})-\mathrm{OAll}$, this depsipeptidyl resin was prepared according to the general procedure described above. Acidolytic cleavage of an aliquot of the resulting resin afforded $\mathrm{C}_{7} \mathrm{H}_{15} \mathrm{CO}-$ Glu-D-Orn-Tyr(O-Ile-Alloc)-D-Ser-Glu-D-Ala-Pro-Gln-D-Tyr-OAll 
in $77 \%$ purity. $\operatorname{HPLC}(\lambda=220 \mathrm{~nm}) t_{R}=7.60 \mathrm{~min} ; \mathrm{MS}(\mathrm{ESI}) \mathrm{m} / \mathrm{z}(+): 732.3[\mathrm{M}+2 \mathrm{H}]^{2+}, 1463.7[\mathrm{M}+\mathrm{H}]^{+}$; MS (ESI) $m / z(-): 1461.6[\mathrm{M}-\mathrm{H}]^{-}, 1483.6[\mathrm{M}+\mathrm{Na}-2 \mathrm{H}]^{-}$.

\section{Linear lipodepsipeptidyl resin $\mathrm{C}_{7} \mathrm{H}_{15} \mathrm{CO}-\mathrm{Glu}\left(\mathrm{O}^{t} \mathrm{Bu}\right)-\mathrm{D}-\mathrm{Orn}(\mathrm{Boc})-\mathrm{D}-\mathrm{Tyr}(\mathrm{O}-\mathrm{Ile}-\mathrm{Alloc})-\mathrm{D}-\mathrm{Thr}\left({ }^{t} \mathrm{Bu}\right)-$} Glu(O'Bu)-D-Val-Pro-Gln(Tr)-Tyr(Wang)-OAll. Starting from $\mathrm{C}_{7} \mathrm{H}_{15} \mathrm{CO}-\mathrm{Glu}\left(\mathrm{O}^{t} \mathrm{Bu}\right)$-D-Orn(Boc)-DTyr-D-Thr $\left({ }^{t} \mathrm{Bu}\right)-\mathrm{Glu}\left(\mathrm{O}^{t} \mathrm{Bu}\right)-\mathrm{D}-$ Val-Pro-Gln(Tr)-Tyr(Wang)-OAll, this depsipeptidyl resin was prepared according to the general procedure described above. Acidolytic cleavage of an aliquot of the resulting resin afforded $\mathrm{C}_{7} \mathrm{H}_{15} \mathrm{CO}-\mathrm{Glu}-\mathrm{D}-\mathrm{Orn}-\mathrm{D}-\mathrm{Tyr}(\mathrm{O}-\mathrm{Ile}-\mathrm{Alloc})$-D-Thr-Glu-D-Val-Pro-Gln-Tyr-OAll in $79 \%$ purity. HPLC $(\lambda=220 \mathrm{~nm}) t_{R}=7.94 \mathrm{~min}$; MS (ESI) $m / z(+): 753.8[\mathrm{M}+2 \mathrm{H}]^{2+}, 1505.8[\mathrm{M}+\mathrm{H}]^{+}$; MS (ESI) $m / z(-): 1503.7[\mathrm{M}-\mathrm{H}]^{-}, 1526.7[\mathrm{M}+\mathrm{Na}-2 \mathrm{H}]^{-}$.

\section{Linear lipodepsipeptidyl resin $\mathrm{C}_{7} \mathrm{H}_{15} \mathrm{CO}-\mathrm{Glu}\left(\mathrm{O}^{t} \mathrm{Bu}\right)$-D-Orn(Boc)-D-Tyr(O-Ile-Alloc)-D-Ser( $\left.{ }^{t} \mathrm{Bu}\right)$ -} Glu(O'Bu)-D-Val-Pro-Gln(Tr)-Tyr(Wang)-OAll. Starting from $\mathrm{C}_{7} \mathrm{H}_{15} \mathrm{CO}-\mathrm{Glu}\left(\mathrm{O}^{t} \mathrm{Bu}\right)$-D-Orn(Boc)-DTyr-D-Ser $\left({ }^{t} \mathrm{Bu}\right)-\mathrm{Glu}\left(\mathrm{O}^{t} \mathrm{Bu}\right)-\mathrm{D}-\mathrm{Val}-\mathrm{Pro}-\mathrm{G} \ln (\mathrm{Tr})-\mathrm{Tyr}(\mathrm{Wang})-\mathrm{OAll}$, this depsipeptidyl resin was prepared according to the general procedure described above. Acidolytic cleavage of an aliquot of the resulting resin afforded $\mathrm{C}_{7} \mathrm{H}_{15} \mathrm{CO}-\mathrm{Glu}-\mathrm{D}-\mathrm{Orn}-\mathrm{D}-\mathrm{Tyr}(\mathrm{O}-\mathrm{Il}$-Alloc)-D-Ser-Glu-D-Val-Pro-Gln-Tyr-OAll in $77 \%$ purity. HPLC $(\lambda=220 \mathrm{~nm}) t_{R}=7.89 \mathrm{~min}$; MS (ESI) $m / z(+): 746.3[\mathrm{M}+2 \mathrm{H}]^{2+}, 1491.7[\mathrm{M}+\mathrm{H}]^{+}$; MS (ESI) $m / z(-): 1489.7[\mathrm{M}-\mathrm{H}]^{-}, 1511.7[\mathrm{M}+\mathrm{Na}-2 \mathrm{H}]^{-}$.

Linear lipodepsipeptidyl resin $\mathrm{C}_{7} \mathrm{H}_{15} \mathrm{CO}-\mathrm{Glu}\left(\mathrm{O}^{t} \mathrm{Bu}\right)-\mathrm{D}-\mathrm{Orn}(\mathrm{Boc})-\mathrm{D}-\mathrm{Ty}(\mathrm{O}-\mathrm{Ile}-\mathrm{Alloc})-\mathrm{D}-\mathrm{Thr}\left({ }^{t} \mathrm{Bu}\right)-$ Glu(O'Bu)-D-Ala-Pro-Gln(Tr)-Tyr(Wang)-OAll. Starting from $\mathrm{C}_{7} \mathrm{H}_{15} \mathrm{CO}-\mathrm{Glu}\left(\mathrm{O}^{t} \mathrm{Bu}\right)$-D-Orn(Boc)-DTyr-D-Thr $\left({ }^{t} \mathrm{Bu}\right)-\mathrm{Glu}\left(\mathrm{O}^{t} \mathrm{Bu}\right)-\mathrm{D}-\mathrm{Ala}-\mathrm{Pro}-\mathrm{Gln}(\mathrm{Tr})-\mathrm{Tyr}($ Wang)-OAll, this depsipeptidyl resin was prepared according to the general procedure described above. Acidolytic cleavage of an aliquot of the resulting resin afforded $\mathrm{C}_{7} \mathrm{H}_{15} \mathrm{CO}-\mathrm{Glu}$-D-Orn-D-Tyr(O-Ile-Alloc)-D-Thr-Glu-D-Ala-Pro-Gln-Tyr-OAll in $83 \%$ 
purity. HPLC $(\lambda=220 \mathrm{~nm}) t_{R}=7.85 \mathrm{~min} ; \mathrm{MS}(\mathrm{ESI}) \mathrm{m} / z(+): 739.8[\mathrm{M}+2 \mathrm{H}]^{2+}, 1477.7[\mathrm{M}+\mathrm{H}]^{+}$; MS (ESI) $m / z(-): 1475.7[\mathrm{M}-\mathrm{H}]^{-}, 1498.6[\mathrm{M}+\mathrm{Na}-2 \mathrm{H}]^{-}$.

\section{Linear lipodepsipeptidyl resin $\mathrm{C}_{7} \mathrm{H}_{15} \mathrm{CO}-\mathrm{Glu}\left(\mathrm{O}^{t} \mathrm{Bu}\right)-\mathrm{D}-\mathrm{Orn}(\mathrm{Boc})-\mathrm{D}-\mathrm{Ty}(\mathrm{O}-\mathrm{Ile}-\mathrm{Alloc})-\mathrm{D}-\mathrm{Ser}\left({ }^{t} \mathrm{Bu}\right)-$} Glu(O'Bu)-D-Ala-Pro-Gln(Tr)-Tyr(Wang)-OAll. Starting from $\mathrm{C}_{7} \mathrm{H}_{15} \mathrm{CO}-\mathrm{Glu}\left(\mathrm{O}^{t} \mathrm{Bu}\right)$-D-Orn(Boc)-DTyr-D-Ser $\left({ }^{t} \mathrm{Bu}\right)-\mathrm{Glu}\left(\mathrm{O}^{t} \mathrm{Bu}\right)-\mathrm{D}-\mathrm{Ala}-\mathrm{Pro}-\mathrm{G} \ln (\mathrm{Tr})-\mathrm{Tyr}(\mathrm{Wang})-\mathrm{OAll}$, this depsipeptidyl resin was prepared according to the general procedure described above. Acidolytic cleavage of an aliquot of the resulting peptidyl resin afforded $\mathrm{C}_{7} \mathrm{H}_{15} \mathrm{CO}-\mathrm{Glu}-\mathrm{D}-\mathrm{Orn}-\mathrm{D}-\mathrm{Tyr}(\mathrm{O}-\mathrm{Ile}-\mathrm{Alloc})$-D-Ser-Glu-D-Ala-Pro-Gln-Tyr-OAll in $83 \%$ purity. $\operatorname{HPLC}(\lambda=220 \mathrm{~nm}) t_{R}=7.79 \mathrm{~min}$; MS (ESI) $\mathrm{m} / \mathrm{z}(+): 732.3[\mathrm{M}+2 \mathrm{H}]^{2+}, 1463.7[\mathrm{M}+\mathrm{H}]^{+}$; MS (ESI) $m / z(-): 1461.7[\mathrm{M}-\mathrm{H}]^{-}, 1483.6[\mathrm{M}+\mathrm{Na}-2 \mathrm{H}]^{-}$.

\section{Linear lipodepsipeptidyl resin $\mathrm{C}_{11} \mathrm{H}_{23} \mathrm{CO}-\mathrm{Glu}\left(\mathrm{O}^{t} \mathrm{Bu}\right)$-D-Orn(Boc)-D-Tyr(O-Ile-Alloc)-D-Thr( $\left.{ }^{t} \mathrm{Bu}\right)$ -} Glu(O'Bu)-D-Val-Pro-Gln(Tr)-Tyr(Wang)-OAll. Starting from $\mathrm{C}_{11} \mathrm{H}_{23} \mathrm{CO}-\mathrm{Glu}\left(\mathrm{O}^{t} \mathrm{Bu}\right)$-D-Orn(Boc)-DTyr-D-Thr( $\left.{ }^{t} \mathrm{Bu}\right)-\mathrm{Glu}\left(\mathrm{O}^{t} \mathrm{Bu}\right)-\mathrm{D}-\mathrm{Val}-\mathrm{Pro}-\mathrm{G} \ln (\mathrm{Tr})-\mathrm{Tyr}(\mathrm{Wang})-\mathrm{OAll}$, this depsipeptidyl resin was prepared according to the general procedure described above. Acidolytic cleavage of an aliquot of the resulting resin afforded $\mathrm{C}_{11} \mathrm{H}_{23} \mathrm{CO}-\mathrm{Glu}-\mathrm{D}-\mathrm{Orn}-\mathrm{D}-\mathrm{Tyr}(\mathrm{O}-\mathrm{Ile}-\mathrm{Alloc}$-D-Thr-Glu-D-Val-Pro-Gln-Tyr-OAll in $82 \%$ purity. HPLC $(\lambda=220 \mathrm{~nm}) t_{R}=9.07 \mathrm{~min}$; MS (ESI) $m / z(+): 1562.0[\mathrm{M}+\mathrm{H}]^{+}, 1583.9[\mathrm{M}+\mathrm{Na}]^{+}$; MS (ESI) $m / z(-): 1559.9[\mathrm{M}-\mathrm{H}]^{-}, 1581.8[\mathrm{M}+\mathrm{Na}-2 \mathrm{H}]^{-}$.

Linear lipodepsipeptidyl resin $\mathrm{C}_{15} \mathrm{H}_{31} \mathrm{CO}-\mathrm{Glu}\left(\mathrm{O}^{t} \mathrm{Bu}\right)-\mathrm{D}-\mathrm{Orn}(\mathrm{Boc})-\mathrm{D}-\mathrm{Tyr}(\mathrm{O}-\mathrm{Ile}-\mathrm{Alloc})-\mathrm{D}-\mathrm{Thr}\left({ }^{t} \mathrm{Bu}\right)-$ Glu(O'Bu)-D-Val-Pro-Gln(Tr)-Tyr(Wang)-OAll. Starting from $\mathrm{C}_{15} \mathrm{H}_{31} \mathrm{CO}-\mathrm{Glu}\left(\mathrm{O}^{t} \mathrm{Bu}\right)$-D-Orn(Boc)-DTyr-D-Thr $\left({ }^{t} \mathrm{Bu}\right)-\mathrm{Glu}\left(\mathrm{O}^{t} \mathrm{Bu}\right)-\mathrm{D}-\mathrm{Val}-\mathrm{Pro}-\mathrm{Gln}(\mathrm{Tr})-\mathrm{Tyr}($ Wang)-OAll, this depsipeptidyl resin was prepared according to the general procedure described above. Acidolytic cleavage of an aliquot of the resulting resin afforded $\mathrm{C}_{15} \mathrm{H}_{31} \mathrm{CO}-\mathrm{Glu}-\mathrm{D}-\mathrm{Orn}-\mathrm{D}-\mathrm{Tyr}(\mathrm{O}-\mathrm{Ile}-\mathrm{Alloc})-\mathrm{D}-\mathrm{Thr}-\mathrm{Glu}-\mathrm{D}-\mathrm{Val}-\mathrm{Pro}-\mathrm{Gln}-\mathrm{Tyr}-\mathrm{OAll}$ in $69 \%$ 
purity. HPLC $(\lambda=220 \mathrm{~nm}) t_{R}=10.13 \mathrm{~min} ; \mathrm{MS}(\mathrm{ESI}) \mathrm{m} / z(+): 1618.0[\mathrm{M}+\mathrm{H}]^{+}, 1639.9[\mathrm{M}+\mathrm{Na}]^{+}$; MS (ESI) $m / z(-): 1616.9[\mathrm{M}-\mathrm{H}]^{-}, 1638.8[\mathrm{M}+\mathrm{Na}-2 \mathrm{H}]^{-}$.

\section{General method for allyl/Alloc removal}

Each peptidyl resin was treated with $\mathrm{Pd}\left(\mathrm{PPh}_{3}\right)_{3}\left(0.1\right.$ equiv) and $\mathrm{PhSiH}_{3}$ (10 equiv) in $\mathrm{CH}_{2} \mathrm{Cl}_{2}$ under nitrogen for $4 \mathrm{~h}$. After this time, the resulting resin was washed with THF $(3 \times 15 \mathrm{sec}), \mathrm{CH}_{2} \mathrm{Cl}_{2}(3 \times 2$ $\min ), \operatorname{DMF}(10 \times 1 \mathrm{~min})$ and $\mathrm{CH}_{2} \mathrm{Cl}_{2}(3 \times 2 \mathrm{~min})$, and air-dried. An aliquot of each resulting peptidyl resin was exposed to acidolytic conditions of TFA/ $\mathrm{H}_{2} \mathrm{O} / \mathrm{TIS}(95: 2.5: 2.5)$ for $2 \mathrm{~h}$ at room temperature. Following TFA evaporation and diethyl ether extraction, the crude peptide was dissolved in $\mathrm{H}_{2} \mathrm{O} / \mathrm{CH}_{3} \mathrm{CN}$, lyophilized, analyzed by HPLC and characterized by mass spectrometry.

\section{Linear depsipeptidyl resin $p N Z-T y r(O-I l e-H)-D-T h r\left({ }^{t} B u\right)-G l u\left(O^{t} B u\right)-D-V a l-P r o-G \ln (T r)-D-$} Tyr(Wang)-OH. This peptidyl resin was obtained from 2 following the above general procedure. Acidolytic cleavage of an aliquot of this resin afforded $p$ NZ-Tyr(O-Ile-H)-D-Thr-Glu-D-Val-Pro-GlnD-Tyr-OH in $72 \%$ purity. HPLC $(\lambda=220 \mathrm{~nm}) t_{R}=6.77 \mathrm{~min}$; MS (ESI) $m / z(+): 615.2[\mathrm{M}+\mathrm{H}+\mathrm{K}]^{2+}$, $1191.5[\mathrm{M}+\mathrm{H}]^{+}, 1213.5[\mathrm{M}+\mathrm{Na}]^{+}, 1229.5[\mathrm{M}+\mathrm{K}]^{+}$.

\section{Linear lipodepsipeptidyl resin $\quad \mathrm{C}_{7} \mathrm{H}_{15} \mathrm{CO}-\mathrm{Glu}\left(\mathrm{O}^{t} \mathrm{Bu}\right)-\mathrm{D}-\mathrm{Orn}(\mathrm{Boc})-\mathrm{Tyr}(\mathrm{O}-\mathrm{Ile}-\mathrm{H})-\mathrm{D}-\mathrm{Th}\left({ }^{t} \mathrm{Bu}\right)-$} Glu(O'Bu)-D-Val-Pro-GIn(Tr)-D-Tyr(Wang)-OH (8a). This peptidyl resin was obtained from 7a following the above general procedure. Acidolytic cleavage of an aliquot of $\mathbf{8 a}$ afforded $\mathrm{C}_{7} \mathrm{H}_{15} \mathrm{CO}-\mathrm{Glu}-$ D-Orn-Tyr(O-Ile-H)-D-Thr-Glu-D-Val-Pro-Gln-D-Tyr-OH (BPC837) in 76\% purity. HPLC ( $\lambda=220$ nm) $t_{R}=6.68 \mathrm{~min} ; \mathrm{MS}(\mathrm{ESI}) \mathrm{m} / z(+): 691.3[\mathrm{M}+2 \mathrm{H}]^{2+}, 1381.6[\mathrm{M}+\mathrm{H}]^{+}$; MS (ESI) $m / z(-): 1379.6[\mathrm{M}-$ $\mathrm{H}]^{-}, 1401.6[\mathrm{M}+\mathrm{Na}-2 \mathrm{H}]^{-}$ 
Linear lipodepsipeptidyl resin $\quad \mathrm{C}_{7} \mathrm{H}_{15} \mathrm{CO}-\mathrm{Glu}\left(\mathrm{O}^{t} \mathrm{Bu}\right)-\mathrm{D}-\mathrm{Orn}(\mathrm{Boc})-\mathrm{Tyr}(\mathrm{O}-\mathrm{Ile}-\mathrm{H})-\mathrm{D}-\mathrm{Ser}\left({ }^{t} \mathrm{Bu}\right)-$ Glu(O'Bu)-D-Val-Pro-GIn(Tr)-D-Tyr(Wang)-OH. This peptidyl resin was obtained from $\mathrm{C}_{7} \mathrm{H}_{15} \mathrm{CO}-$ Glu( $\left.{ }^{t} \mathrm{Bu}\right)-\mathrm{D}-\mathrm{Orn}(\mathrm{Boc})-\mathrm{Tyr}(\mathrm{O}-\mathrm{Ile}-\mathrm{Alloc})-\mathrm{D}-\mathrm{Ser}\left({ }^{t} \mathrm{Bu}\right)-\mathrm{Glu}\left(\mathrm{O}^{t} \mathrm{Bu}\right)-\mathrm{D}-\mathrm{Val}-\mathrm{Pro}-\mathrm{G} \ln (\mathrm{Tr})-\mathrm{D}-\mathrm{Tyr}(\mathrm{Wang})-\mathrm{OAll}$ following the above general procedure. Acidolytic cleavage of an aliquot of the resulting resin afforded $\mathrm{C}_{7} \mathrm{H}_{15} \mathrm{CO}-\mathrm{Glu}-\mathrm{D}-\mathrm{Orn}-\mathrm{Tyr}(\mathrm{O}-\mathrm{Il}-\mathrm{H})-\mathrm{D}-\mathrm{Ser}-\mathrm{Glu}-\mathrm{D}-\mathrm{V}$ al-Pro-Gln-D-Tyr-OH (BPC839) in 86\% purity. $\operatorname{HPLC}(\lambda=220 \mathrm{~nm}) t_{R}=6.65 \mathrm{~min} ; \mathrm{MS}(\mathrm{ESI}) \mathrm{m} / \mathrm{z}(+): 684.3[\mathrm{M}+2 \mathrm{H}]^{2+}, 1367.6[\mathrm{M}+\mathrm{H}]^{+} ; \mathrm{MS}(\mathrm{ESI})$ $m / z(-): 1365.6[\mathrm{M}-\mathrm{H}]^{-}, 1387.6[\mathrm{M}+\mathrm{Na}-2 \mathrm{H}]^{-}$.

Linear lipodepsipeptidyl resin $\quad \mathrm{C}_{7} \mathrm{H}_{15} \mathrm{CO}-\mathrm{Glu}\left(\mathrm{O}^{t} \mathrm{Bu}\right)-\mathrm{D}-\mathrm{Orn}(\mathrm{Boc})-\mathrm{Tyr}(\mathrm{O}-\mathrm{Ile}-\mathrm{H})-\mathrm{D}-\mathrm{Thr}\left({ }^{t} \mathrm{Bu}\right)-$ Glu(Ot ${ }^{t}$ Bu)-D-Ala-Pro-Gln(Tr)-D-Tyr(Wang)-OH. This peptidyl resin was obtained from $\mathrm{C}_{7} \mathrm{H}_{15} \mathrm{CO}$ Glu(O $\left.{ }^{t} \mathrm{Bu}\right)-\mathrm{D}-\mathrm{Orn}(\mathrm{Boc})-\mathrm{Tyr}(\mathrm{O}-\mathrm{Ile}-\mathrm{Alloc})-\mathrm{D}-\mathrm{Thr}\left({ }^{t} \mathrm{Bu}\right)-\mathrm{Glu}\left(\mathrm{O}^{t} \mathrm{Bu}\right)-\mathrm{D}-\mathrm{Ala}-\mathrm{Pro}-\mathrm{Gln}(\mathrm{Tr})-\mathrm{D}-\mathrm{Tyr}(\mathrm{Wang})-$ OAll following the above general procedure. Acidolytic cleavage of an aliquot of the resulting resin afforded $\mathrm{C}_{7} \mathrm{H}_{15} \mathrm{CO}-\mathrm{Glu}-\mathrm{D}-\mathrm{Orn}-\mathrm{Tyr}(\mathrm{O}-\mathrm{Ile}-\mathrm{H})-\mathrm{D}-\mathrm{Thr}-\mathrm{Glu}-\mathrm{D}-\mathrm{Ala}-\mathrm{Pro}-\mathrm{Gln}-\mathrm{D}-\mathrm{Tyr}-\mathrm{OH}$ (BPC841) in $82 \%$ purity. HPLC $(\lambda=220 \mathrm{~nm}) t_{R}=6.59 \mathrm{~min}$; MS (ESI) $m / z(+): 677.3[\mathrm{M}+2 \mathrm{H}]^{2+}, 1353.6[\mathrm{M}+\mathrm{H}]^{+}$; MS (ESI) $m / z(-): 1351.6[\mathrm{M}-\mathrm{H}]^{-}, 1373.5[\mathrm{M}+\mathrm{Na}-2 \mathrm{H}]^{-}$.

\section{Linear lipodepsipeptidyl resin $\quad \mathrm{C}_{7} \mathrm{H}_{15} \mathrm{CO}-\mathrm{Glu}\left(\mathrm{O}^{t} \mathrm{Bu}\right)-\mathrm{D}-\mathrm{Orn}(\mathrm{Boc})-\mathrm{Ty}(\mathrm{O}-\mathrm{Ile}-\mathrm{H})-\mathrm{D}-\mathrm{Ser}\left({ }^{t} \mathrm{Bu}\right)-$} Glu(O'Bu)-D-Ala-Pro-GIn(Tr)-D-Tyr(Wang)-OH. This peptidyl resin was obtained from $\mathrm{C}_{7} \mathrm{H}_{15} \mathrm{CO}-$ $\mathrm{Glu}\left(\mathrm{O}^{t} \mathrm{Bu}\right)-\mathrm{D}-\mathrm{Orn}(\mathrm{Boc})-\mathrm{Tyr}(\mathrm{O}-\mathrm{Ile}-\mathrm{Alloc})-\mathrm{D}-\mathrm{Ser}\left({ }^{t} \mathrm{Bu}\right)-\mathrm{Glu}\left(\mathrm{O}^{t} \mathrm{Bu}\right)-\mathrm{D}-\mathrm{Ala}-\mathrm{Pro}-\mathrm{G} \ln (\mathrm{Tr})-\mathrm{D}-\mathrm{Tyr}(\mathrm{Wang})-\mathrm{OAll}$ following the above general procedure. Acidolytic cleavage of an aliquot of the resulting resin afforded $\mathrm{C}_{7} \mathrm{H}_{15} \mathrm{CO}-\mathrm{Glu}-\mathrm{D}-\mathrm{Orn}-\mathrm{Tyr}(\mathrm{O}-\mathrm{Ile}-\mathrm{H})-\mathrm{D}-\mathrm{Ser}-\mathrm{Glu}-\mathrm{D}-\mathrm{Ala}-\mathrm{Pro}-\mathrm{Gln}-\mathrm{D}-\mathrm{Tyr}-\mathrm{OH}$ (BPC843) in 69\% purity. $\operatorname{HPLC}(\lambda=220 \mathrm{~nm}) t_{R}=6.55 \mathrm{~min} ; \mathrm{MS}(\mathrm{ESI}) \mathrm{m} / \mathrm{z}(+): 670.3[\mathrm{M}+2 \mathrm{H}]^{2+}, 1339.6[\mathrm{M}+\mathrm{H}]^{+} ; \mathrm{MS}(\mathrm{ESI})$ $m / z(-): 1337.6[\mathrm{M}-\mathrm{H}]^{-}, 1359.6[\mathrm{M}+\mathrm{Na}-2 \mathrm{H}]^{-}$. 
Linear lipodepsipeptidyl resin $\mathrm{C}_{7} \mathrm{H}_{15} \mathrm{CO}-\mathrm{Glu}\left(\mathrm{O}^{t} \mathrm{Bu}\right)-\mathrm{D}-\mathrm{Orn}(\mathrm{Boc})-\mathrm{D}-\mathrm{Tyr}(\mathrm{O}-\mathrm{Ile}-\mathrm{H})-\mathrm{D}-\mathrm{Thr}\left({ }^{t} \mathrm{Bu}\right)-$ Glu(Ot $\left.{ }^{t} \mathbf{B u}\right)$-D-Val-Pro-GIn(Tr)-Tyr(Wang)-OH. This peptidyl resin was obtained from $\mathrm{C}_{7} \mathrm{H}_{15} \mathrm{CO}-$ Glu(O $\left.{ }^{t} \mathrm{Bu}\right)-\mathrm{D}-\mathrm{Orn}(\mathrm{Boc})-\mathrm{D}-\mathrm{Tyr}(\mathrm{O}-\mathrm{Ile}-\mathrm{Alloc})-\mathrm{D}-\mathrm{Thr}\left({ }^{t} \mathrm{Bu}\right)-\mathrm{Glu}\left(\mathrm{O}^{t} \mathrm{Bu}\right)-\mathrm{D}-\mathrm{Val}-\mathrm{Pro}-\mathrm{Gln}(\mathrm{Tr})-\mathrm{Tyr}(\mathrm{Wang})-$

OAll following the above general procedure. Acidolytic cleavage of an aliquot of the resulting resin afforded $\mathrm{C}_{7} \mathrm{H}_{15} \mathrm{CO}-\mathrm{Glu}-\mathrm{D}-\mathrm{Orn}-\mathrm{D}-\mathrm{Tyr}(\mathrm{O}-\mathrm{Ile}-\mathrm{H})-\mathrm{D}-\mathrm{Thr}-\mathrm{Glu}-\mathrm{D}-\mathrm{Val}-\mathrm{Pro}-\mathrm{Gln}-\mathrm{Tyr}-\mathrm{OH}$ (BPC845) in $87 \%$ purity. HPLC $(\lambda=220 \mathrm{~nm}) t_{R}=6.82 \mathrm{~min}$; MS (ESI) $\mathrm{m} / z(+): 1268.6[\mathrm{M}-\mathrm{Ile}+\mathrm{H}]^{+}, 1381.7[\mathrm{M}+\mathrm{H}]^{+} ; \mathrm{MS}$ (ESI) $m / z(-): 1266.6[\mathrm{M}-\mathrm{Ile}-\mathrm{H}]^{-}, 1379.6[\mathrm{M}-\mathrm{H}]^{-}, 1401.6[\mathrm{M}+\mathrm{Na}-2 \mathrm{H}]^{-}$.

\section{Linear lipodepsipeptidyl resin $\mathrm{C}_{7} \mathrm{H}_{15} \mathrm{CO}-\mathrm{Glu}\left(\mathrm{O}^{t} \mathrm{Bu}\right)-\mathrm{D}-\mathrm{Orn}(\mathrm{Boc})-\mathrm{D}-\mathrm{Tyr}(\mathrm{O}-\mathrm{Ile}-\mathrm{H})-\mathrm{D}-\mathrm{Ser}\left({ }^{t} \mathrm{Bu}\right)$ -} Glu(Ot ${ }^{t}$ Bu)-D-Val-Pro-Gln(Tr)-Tyr(Wang)-OH. This peptidyl resin was obtained from $\mathrm{C}_{7} \mathrm{H}_{15} \mathrm{CO}$ Glu(O $\left.{ }^{t} \mathrm{Bu}\right)-\mathrm{D}-\mathrm{Orn}(\mathrm{Boc})-\mathrm{D}-\mathrm{Tyr}(\mathrm{O}-\mathrm{Ile}-\mathrm{Alloc})-\mathrm{D}-\mathrm{Ser}\left({ }^{t} \mathrm{Bu}\right)-\mathrm{Glu}\left(\mathrm{O}^{t} \mathrm{Bu}\right)-\mathrm{D}-\mathrm{Val}-\mathrm{Pro}-\mathrm{G} \ln (\mathrm{Tr})-\mathrm{Tyr}(\mathrm{Wang})-\mathrm{OAll}$ following the above general procedure. Acidolytic cleavage of an aliquot of the resulting resin afforded $\mathrm{C}_{7} \mathrm{H}_{15} \mathrm{CO}-\mathrm{Glu}-\mathrm{D}-\mathrm{Orn}-\mathrm{D}-\mathrm{Tyr}(\mathrm{O}-\mathrm{Ile}-\mathrm{H})-\mathrm{D}-\mathrm{Ser}-\mathrm{Glu}-\mathrm{D}-\mathrm{Val}-\mathrm{Pro}-\mathrm{Gln}-\mathrm{Tyr}-\mathrm{OH}$ (BPC847) in 93\% purity. $\operatorname{HPLC}(\lambda=220 \mathrm{~nm}) t_{R}:=6.78 \mathrm{~min} ; \mathrm{MS}(\mathrm{ESI}) \mathrm{m} / z(+): 1254.6[\mathrm{M}-\mathrm{Ile}+\mathrm{H}]^{+}, 1367.7[\mathrm{M}+\mathrm{H}]^{+} ; \mathrm{MS}(\mathrm{ESI})$ $m / z(-): 1252.5$ [M-Ile-H]', $1365.6[\mathrm{M}-\mathrm{H}]^{-}, 1387.6[\mathrm{M}+\mathrm{Na}-2 \mathrm{H}]^{-}$.

\section{Linear lipodepsipeptidyl resin $\mathrm{C}_{7} \mathrm{H}_{15} \mathrm{CO}-\mathrm{Glu}\left(\mathrm{O}^{t} \mathrm{Bu}\right)-\mathrm{D}-\mathrm{Orn}(\mathrm{Boc})-\mathrm{D}-\mathrm{Tyr}(\mathrm{O}-\mathrm{Ile}-\mathrm{H})-\mathrm{D}-\mathrm{Th}\left({ }^{t} \mathrm{Bu}\right)-$} Glu(Ot $\left.{ }^{t} \mathbf{B u}\right)$-D-Ala-Pro-GIn(Tr)-Tyr(Wang)-OH. This peptidyl resin was obtained from $\mathrm{C}_{7} \mathrm{H}_{15} \mathrm{CO}$ Glu(O $\left.{ }^{t} \mathrm{Bu}\right)-\mathrm{D}-\mathrm{Orn}(\mathrm{Boc})-\mathrm{D}-\mathrm{Tyr}(\mathrm{O}-\mathrm{Ile}-\mathrm{Alloc})-\mathrm{D}-\mathrm{Thr}\left({ }^{t} \mathrm{Bu}\right)-\mathrm{Glu}\left(\mathrm{O}^{t} \mathrm{Bu}\right)$-D-Ala-Pro-Gln(Tr)-Tyr(Wang)OAll following the above general procedure. Acidolytic cleavage of an aliquot of the resulting resin afforded $\mathrm{C}_{7} \mathrm{H}_{15} \mathrm{CO}-\mathrm{Glu}-\mathrm{D}-\mathrm{Orn}-\mathrm{D}-\mathrm{Tyr}(\mathrm{O}-\mathrm{Ile}-\mathrm{H})-\mathrm{D}-\mathrm{Thr}-\mathrm{Glu}-\mathrm{D}-\mathrm{Ala}-\mathrm{Pro}-\mathrm{Gln}-\mathrm{Tyr}-\mathrm{OH}$ (BPC849) in 79\% purity. HPLC $(\lambda=220 \mathrm{~nm}) t_{R}=6.73 \mathrm{~min}$; MS (ESI) $m / z(+): 677.4[\mathrm{M}+2 \mathrm{H}]^{2+}, 1240.6[\mathrm{M}-\mathrm{Ile}+\mathrm{H}]^{+}$, $1353.7[\mathrm{M}+\mathrm{H}]^{+}$; MS (ESI) m/z(-): 1238.5 [M-Ile-H]', $1351.6[\mathrm{M}-\mathrm{H}]^{-}, 1373.6[\mathrm{M}+\mathrm{Na}-2 \mathrm{H}]^{-}$. 
Linear lipodepsipeptidyl resin $\mathrm{C}_{7} \mathrm{H}_{15} \mathrm{CO}-\mathrm{Glu}\left(\mathrm{O}^{t} \mathrm{Bu}\right)-\mathrm{D}-\mathrm{Orn}(\mathrm{Boc})-\mathrm{D}-\mathrm{Tyr}(\mathrm{O}-\mathrm{Ile}-\mathrm{H})-\mathrm{D}-\mathrm{Ser}\left({ }^{t} \mathrm{Bu}\right)-$ Glu(O'Bu)-D-Ala-Pro-GIn(Tr)-Tyr(Wang)-OH. This peptidyl resin was obtained from $\mathrm{C}_{7} \mathrm{H}_{15} \mathrm{CO}-$ Glu( $\left.{ }^{t} \mathrm{Bu}\right)$-D-Orn(Boc)-D-Tyr(O-Ile-Alloc)-D-Ser( $\left.{ }^{t} \mathrm{Bu}\right)-\mathrm{Glu}\left(\mathrm{O}^{t} \mathrm{Bu}\right)-\mathrm{D}-\mathrm{Ala}-\mathrm{Pro}-\mathrm{G} \ln (\mathrm{Tr})-\mathrm{Tyr}(\mathrm{Wang})-\mathrm{OAll}$ following the above general procedure. Acidolytic cleavage of an aliquot of the resulting resin afforded $\mathrm{C}_{7} \mathrm{H}_{15} \mathrm{CO}-\mathrm{Glu}-\mathrm{D}-\mathrm{Orn}-\mathrm{D}-\mathrm{Tyr}(\mathrm{O}-\mathrm{Ile}-\mathrm{H})-\mathrm{D}-\mathrm{Ser}-\mathrm{Glu}-\mathrm{D}-\mathrm{Ala}-\mathrm{Pro}-\mathrm{Gln}-\mathrm{Tyr}-\mathrm{OH}$ (BPC851) in 78\% purity. $\operatorname{HPLC}(\lambda=220 \mathrm{~nm}) t_{R}=6.67 \mathrm{~min} ; \mathrm{MS}(\mathrm{ESI}) \mathrm{m} / z(+): 1226.6[\mathrm{M}-\mathrm{Ile}+\mathrm{H}]^{+}, 1339.7[\mathrm{M}+\mathrm{H}]^{+}, 1361.6$ $[\mathrm{M}+\mathrm{Na}]^{+}$; MS (ESI) m/z(-): $1224.5[\mathrm{M}-\mathrm{Ile}-\mathrm{H}]^{-}, 1337.6[\mathrm{M}-\mathrm{H}]^{-}, 1359.6[\mathrm{M}+\mathrm{Na}-2 \mathrm{H}]^{-}$.

\section{Linear lipodepsipeptidyl resin $\mathrm{C}_{11} \mathrm{H}_{23} \mathrm{CO}-\mathrm{Glu}\left(\mathrm{O}^{t} \mathrm{Bu}\right)-\mathrm{D}-\mathrm{Orn}(\mathrm{Boc})-\mathrm{Tyr}(\mathrm{O}-\mathrm{Ile}-\mathrm{H})-\mathrm{D}-\mathrm{Thr}\left({ }^{t} \mathrm{Bu}\right)-$} Glu(Ot ${ }^{t}$ u) $)$-D-Val-Pro-Gln(Tr)-D-Tyr(Wang)-OH (8b). This peptidyl resin was obtained from $7 \mathbf{b}$ following the above general procedure. Acidolytic cleavage of an aliquot of $\mathbf{8 b}$ afforded $\mathrm{C}_{11} \mathrm{H}_{23} \mathrm{CO}$ Glu-D-Orn-Tyr(O-Ile-H)-D-Thr-Glu-D-Val-Pro-Gln-D-Tyr-OH (BPC853) in 86\% purity. HPLC $(\lambda=$ $220 \mathrm{~nm}) t_{R}=7.45 \mathrm{~min} ; \mathrm{MS}(\mathrm{ESI}) \mathrm{m} / z(+): 719.4[\mathrm{M}+2 \mathrm{H}]^{2+}, 1437.7[\mathrm{M}+\mathrm{H}]^{+}$; MS (ESI) $\mathrm{m} / z(-): 1436.6$ $[\mathrm{M}-\mathrm{H}]^{-}, 1457.6[\mathrm{M}+\mathrm{Na}-2 \mathrm{H}]^{-}$

Linear lipodepsipeptidyl resin $\mathrm{C}_{15} \mathrm{H}_{31} \mathrm{CO}-\mathrm{Glu}\left(\mathrm{O}^{t} \mathrm{Bu}\right)-\mathrm{D}-\mathrm{Orn}(\mathrm{Boc})-\mathrm{Tyr}(\mathrm{O}-\mathrm{Ile}-\mathrm{H})-\mathrm{D}-\mathrm{Thr}\left({ }^{t} \mathrm{Bu}\right)-$ Glu(O'Bu)-D-Val-Pro-GIn(Tr)-D-Tyr(Wang)-OH (8c). This peptidyl resin was obtained from 7c following the above general procedure. Acidolytic cleavage of an aliquot of $\mathbf{8 c}$ afforded $\mathrm{C}_{15} \mathrm{H}_{31} \mathrm{CO}$ Glu-D-Orn-Tyr(O-Ile-H)-D-Thr-Glu-D-Val-Pro-Gln-D-Tyr-OH (BPC855) in 89\% purity. HPLC $(\lambda=$ $220 \mathrm{~nm}) t_{R}=8.23 \mathrm{~min} ; \mathrm{MS}(\mathrm{ESI}) \mathrm{m} / \mathrm{z}(+): 747.4[\mathrm{M}+2 \mathrm{H}]^{2+}, 1493.8[\mathrm{M}+\mathrm{H}]^{+}$; MS (ESI) $\mathrm{m} / z(-): 1491.7$ $[\mathrm{M}-\mathrm{H}]^{-}, 1513.7[\mathrm{M}+\mathrm{Na}-2 \mathrm{H}]^{-}$

Linear lipodepsipeptidyl resin $\mathrm{C}_{11} \mathrm{H}_{23} \mathrm{CO}-\mathrm{Glu}\left(\mathrm{O}^{t} \mathrm{Bu}\right)-\mathrm{D}-\mathrm{Orn}(\mathrm{Boc})-\mathrm{D}-\mathrm{Tyr}(\mathrm{O}-\mathrm{Ile}-\mathrm{H})-\mathrm{D}-\mathrm{Thr}\left({ }^{t} \mathrm{Bu}\right)-$ Glu(O'Bu)-D-Val-Pro-GIn(Tr)-Tyr(Wang)-OH. This peptidyl resin was obtained from $\mathrm{C}_{11} \mathrm{H}_{23} \mathrm{CO}$ Glu(O $\left.{ }^{t} \mathrm{Bu}\right)-\mathrm{D}-\mathrm{Orn}(\mathrm{Boc})-\mathrm{D}-\mathrm{Tyr}(\mathrm{O}-\mathrm{Ile}-\mathrm{Alloc})-\mathrm{D}-\mathrm{Thr}\left({ }^{t} \mathrm{Bu}\right)-\mathrm{Glu}\left(\mathrm{O}^{t} \mathrm{Bu}\right)-\mathrm{D}-\mathrm{Val}-\mathrm{Pro}-\mathrm{Gln}(\mathrm{Tr})-\mathrm{Tyr}(\mathrm{Wang})-$ 
OAll following the above general procedure. Acidolytic cleavage of an aliquot of the resulting resin afforded $\mathrm{C}_{11} \mathrm{H}_{23} \mathrm{CO}-\mathrm{Glu}-\mathrm{D}-\mathrm{Orn}-\mathrm{D}-\mathrm{Tyr}(\mathrm{O}-\mathrm{Ile}-\mathrm{H})-\mathrm{D}-\mathrm{Thr}-\mathrm{Glu}-\mathrm{D}-\mathrm{Val}-\mathrm{Pro}-\mathrm{Gln}-\mathrm{Tyr}-\mathrm{OH}$ (BPC857) in 62\% purity. HPLC $(\lambda=220 \mathrm{~nm}) t_{R}=7.84 \mathrm{~min} ; \mathrm{MS}(\mathrm{ESI}) \mathrm{m} / z(+): 719.4[\mathrm{M}+2 \mathrm{H}]^{2+}, 1325.8[\mathrm{M}-\mathrm{Ile}+\mathrm{H}]^{+}$, $1437.9[\mathrm{M}+\mathrm{H}]^{+}, 1459.9[\mathrm{M}+\mathrm{Na}]^{+}$; MS (ESI) m/z(-): $1322.7[\mathrm{M}-\mathrm{Ile}-\mathrm{H}]^{-}, 1435.8[\mathrm{M}-\mathrm{H}]^{-}, 1457.8[\mathrm{M}+\mathrm{Na}-$ $2 \mathrm{H}]^{-}$.

\section{Linear lipodepsipeptidyl resin $\mathrm{C}_{15} \mathrm{H}_{31} \mathrm{CO}-\mathrm{Glu}\left(\mathrm{O}^{t} \mathrm{Bu}\right)$-D-Orn(Boc)-D-Tyr(O-Ile-H)-D-Thr( $\left.{ }^{t} \mathrm{Bu}\right)$ -} Glu(O'Bu)-D-Val-Pro-GIn(Tr)-Tyr(Wang)-OH. This peptidyl resin was obtained from $\mathrm{C}_{15} \mathrm{H}_{31} \mathrm{CO}-$ Glu(O $\left.{ }^{t} \mathrm{Bu}\right)-\mathrm{D}-\mathrm{Orn}(\mathrm{Boc})-\mathrm{D}-\mathrm{Tyr}(\mathrm{O}-\mathrm{Ile}-\mathrm{Alloc})-\mathrm{D}-\mathrm{Thr}\left({ }^{t} \mathrm{Bu}\right)-\mathrm{Glu}\left(\mathrm{O}^{t} \mathrm{Bu}\right)-\mathrm{D}-\mathrm{Val}-\mathrm{Pro}-\mathrm{Gln}(\mathrm{Tr})-\mathrm{Tyr}(\mathrm{Wang})-$ OAll following the above general procedure. Acidolytic cleavage of an aliquot of the resulting resin afforded $\mathrm{C}_{15} \mathrm{H}_{31} \mathrm{CO}-\mathrm{Glu}-\mathrm{D}-\mathrm{Orn}-\mathrm{D}-\mathrm{Tyr}(\mathrm{O}-\mathrm{Ile}-\mathrm{H})-\mathrm{D}-\mathrm{Thr}-\mathrm{Glu}-\mathrm{D}-\mathrm{Val}-\mathrm{Pro}-\mathrm{Gln}-\mathrm{Tyr}-\mathrm{OH}$ (BPC859) in 64\% purity. HPLC $(\lambda=220 \mathrm{~nm}) t_{R}=8.69 \mathrm{~min} ; \mathrm{MS}(\mathrm{ESI}) \mathrm{m} / z(+): 747.5[\mathrm{M}+2 \mathrm{H}]^{2+}, 758.4[\mathrm{M}+\mathrm{Na}+\mathrm{H}]^{2+}$, $1380.9[\mathrm{M}-\mathrm{Ile}+\mathrm{H}]^{+}, 1494.0[\mathrm{M}+\mathrm{H}]^{+}, 1516.9[\mathrm{M}+\mathrm{Na}]^{+}$; MS (ESI) $m / z(-): 1378.8[\mathrm{M}-\mathrm{Ile}+\mathrm{H}]^{-}, 1491.9[\mathrm{M}-$ $\mathrm{H}]^{-}, 1513.9[\mathrm{M}+\mathrm{Na}-2 \mathrm{H}]^{-}$.

\section{Synthesis of the cyclic lipodepsipeptides}

On-resin cyclization of each linear peptidyl resin was mediated by Oxyma (5 equiv), [ethyl cyano(hydroxyimino)acetato- $\left.O^{2}\right] \operatorname{tri}$-(1-pyrrolidinyl)-phosphonium hexafluorophosphate (PyOxim) (5 equiv) and DIEA (10 equiv) in DMF. The reaction mixture was stirred at room temperature for $24 \mathrm{~h}$. The resin was then washed with DMF $(6 \times 1 \mathrm{~min}), \mathrm{CH}_{2} \mathrm{Cl}_{2}(3 \times 1 \mathrm{~min})$ and diethyl ether $(3 \times 2 \mathrm{~min})$, and dried in vacuo. The completion of the cyclization was checked with the Kaiser test. ${ }^{45}$ The resulting peptide was cleaved from the resin with TFA/ $\mathrm{H}_{2} \mathrm{O} / \mathrm{TIS}(95: 2.5: 2.5)$ for $2 \mathrm{~h}$ at room temperature. 
Following TFA evaporation and diethyl ether extraction, the crude peptide was dissolved in $\mathrm{H}_{2} \mathrm{O} / \mathrm{CH}_{3} \mathrm{CN}$, lyophilized and analyzed by HPLC and mass spectrometry.

Cyclic depsipeptide $p$ NZ-Tyr(\&)-D-Thr-Glu-D-Val-Pro-Gln-D-Tyr-Ile-\& (4). Starting from $p$ NZTyr(O-Ile-H)-D-Thr( $\left.{ }^{t} \mathrm{Bu}\right)-\mathrm{Glu}\left(\mathrm{O}^{t} \mathrm{Bu}\right)-\mathrm{D}-\mathrm{Val}-\mathrm{Pro}-\mathrm{G} \ln (\mathrm{Tr})-\mathrm{D}-\mathrm{Tyr}(\mathrm{Wang})-\mathrm{OH}, \quad$ on-resin $\quad$ cyclization followed by acidolytic cleavage afforded the cyclic depsipeptide $p$ NZ-Tyr(\&)-D-Thr-Glu-D-Val-ProGln-D-Tyr-Ile- $\&$ (4) in $31 \%$ purity. HPLC $(\lambda=220 \mathrm{~nm}) t_{R}=7.55 \mathrm{~min}$; MS (ESI) $\mathrm{m} / z(+): 1195.5$ $[\mathrm{M}+\mathrm{Na}]^{+}, 1211.4[\mathrm{M}+\mathrm{K}]^{+}$; MS (ESI) $m / z(-): 1171.5[\mathrm{M}-\mathrm{H}]^{-}$.

\section{Cyclic lipodepsipeptide $\quad \mathrm{C}_{7} \mathrm{H}_{15} \mathrm{CO}-$ Glu-D-Orn-Tyr(\&)-D-Thr-Glu-D-Val-Pro-Gln-D-Tyr-Ile-\&}

(BPC838). Starting from 8a, on-resin cyclization followed by acidolytic cleavage afforded the cyclic lipodepsipeptide BPC838 in 33\% purity. $\operatorname{HPLC}(\lambda=220 \mathrm{~nm}) t_{R}=7.26 \mathrm{~min}$; MS (ESI) $\mathrm{m} / z(+): 1363.6$ $[\mathrm{M}+\mathrm{H}]^{+}$; MS (ESI) $m / z(-)$ : $1361.6[\mathrm{M}-\mathrm{H}]^{-}$. The crude peptide was purified by RP-HPLC obtaining BPC838 in $>99 \%$ purity. HPLC $(\lambda=220 \mathrm{~nm}) t_{R}=7.45 \mathrm{~min} ;{ }^{1} \mathrm{H}-\mathrm{NMR}\left(400 \mathrm{MHz}, \mathrm{DMSO}-\mathrm{d}_{6}+\mathrm{D}_{2} \mathrm{O}\right) \delta$ (ppm): 0.72-0.87 (m, $18 \mathrm{H}), 1.15-1.20(\mathrm{~m}, 14 \mathrm{H}), 1.41-1.45(\mathrm{~m}, 4 \mathrm{H}), 1.55-1.60$ (m, $3 \mathrm{H}), 1.69-1.76$ (m, $4 \mathrm{H}), 1.82-1.98(\mathrm{~m}, 4 \mathrm{H}), 2.06-2.26(\mathrm{~m}, 10 \mathrm{H}), 2.67-2.77$ (m, $3 \mathrm{H}), 2.86-2.88(\mathrm{~m}, 2 \mathrm{H}), 3.16-3.27$ (m, 2 H), 3.50-3.53 (m, $1 \mathrm{H}), 3.97-4.00(\mathrm{~m}, 1 \mathrm{H}), 4.17-4.26(\mathrm{~m}, 3 \mathrm{H}), 4.31-4.39$ (m, $1 \mathrm{H}), 4.44-4.47$ (m, $1 \mathrm{H})$, 4.55-4.63 (m, $2 \mathrm{H}), 6.63$ (d, $2 \mathrm{H}, J=8.0 \mathrm{~Hz}), 6.84(\mathrm{~d}, 2 \mathrm{H}, J=8.0 \mathrm{~Hz}), 6.99-7.04(\mathrm{~m}, 4 \mathrm{H})$; MS (ESI) $m / z(+): 682.3[\mathrm{M}+2 \mathrm{H}]^{2+}, 693.3[\mathrm{M}+\mathrm{Na}+\mathrm{H}]^{2+}, 1363.8[\mathrm{M}+\mathrm{H}]^{+}, 1385.8[\mathrm{M}+\mathrm{Na}]^{+} ; \mathrm{MS}(\mathrm{ESI}) m / z(-):$ $1361.8[\mathrm{M}-\mathrm{H}]^{-}, 1383.6[\mathrm{M}+\mathrm{Na}-2 \mathrm{H}]^{-}$; HRMS (ESI) $m / z(+)$ : calcd for $\mathrm{C}_{66} \mathrm{H}_{99} \mathrm{~N}_{12} \mathrm{O}_{19}$ 1363.7144, found 1363.7130; calcd for $\mathrm{C}_{66} \mathrm{H}_{98} \mathrm{~N}_{12} \mathrm{NaO}_{19}$ 1385.6963, found 1385.6934; calcd for $\mathrm{C}_{66} \mathrm{H}_{99} \mathrm{~N}_{12} \mathrm{NaO}_{19}$ 693.3518, found 693.3505; calcd for $\mathrm{C}_{66} \mathrm{H}_{98} \mathrm{~N}_{12} \mathrm{Na}_{2} \mathrm{O}_{19}$ 704.3428, found 704.3408.

\section{Cyclic lipodepsipeptide $\quad \mathrm{C}_{7} \mathrm{H}_{15} \mathrm{CO}$-Glu-D-Orn-Tyr(\&)-D-Ser-Glu-D-Val-Pro-Gln-D-Tyr-Ile-\&} (BPC840). Starting from $\mathrm{C}_{7} \mathrm{H}_{15} \mathrm{CO}-\mathrm{Glu}\left(\mathrm{O}^{t} \mathrm{Bu}\right)$-D-Orn(Boc)-Tyr(O-Ile-H)-D-Ser( $\left.{ }^{t} \mathrm{Bu}\right)-\mathrm{Glu}\left(\mathrm{O}^{t} \mathrm{Bu}\right)-\mathrm{D}-\mathrm{Val}-$ 
Pro-Gln(Tr)-D-Tyr(Wang)-OH, on-resin cyclization followed by acidolytic cleavage afforded the cyclic lipodepsipeptide BPC840 in 35\% purity. $\operatorname{HPLC}(\lambda=220 \mathrm{~nm}) t_{R}=7.25 \mathrm{~min}$; MS (ESI) $\mathrm{m} / z(+): 1349.6$ $[\mathrm{M}+\mathrm{H}]^{+}$; MS (ESI) $m / z(-)$ : $1347.6[\mathrm{M}-\mathrm{H}]^{-}$. The crude peptide was purified by RP-HPLC obtaining BPC840 in $>99 \%$ purity. HPLC $(\lambda=220 \mathrm{~nm}) t_{R}=7.22 \mathrm{~min} ;{ }^{1} \mathrm{H}-\mathrm{NMR}\left(400 \mathrm{MHz}, \mathrm{DMSO}-\mathrm{d}_{6}+\mathrm{D}_{2} \mathrm{O}\right) \delta$ (ppm): 0.73-0.86 (m, $15 \mathrm{H}), 1.14-1.21(\mathrm{~m}, 12 \mathrm{H}), 1.41-1.48(\mathrm{~m}, 4 \mathrm{H}), 1.55-1.61(\mathrm{~m}, 3 \mathrm{H}), 1.68-1.90$ (m, $10 \mathrm{H}), 2.00-2.33$ (m, 8 H), 2.67-2.77 (m, 2 H), 2.85-2.87 (m, 2 H), 3.19-3.33 (m, 4 H), 3.97-3.99 (m, 1 H), 4.12-4.26 (m, $6 \mathrm{H}), 4.35-4.46(\mathrm{~m}, 2 \mathrm{H}), 4.56-4.60(\mathrm{~m}, 1 \mathrm{H}), 4.64-4.75(\mathrm{~m}, 1 \mathrm{H}), 6.63(\mathrm{~d}, 2 \mathrm{H}, J=$ $8.0 \mathrm{~Hz}), 6.86(\mathrm{~d}, 2 \mathrm{H}, J=8.0 \mathrm{~Hz}), 6.95-7.04(\mathrm{~m}, 4 \mathrm{H})$; MS (ESI) $m / z(+): 675.3[\mathrm{M}+2 \mathrm{H}]^{2+}, 1349.7$ $[\mathrm{M}+\mathrm{H}]^{+}$; MS (ESI) $m / z(-)$ : $1347.6[\mathrm{M}-\mathrm{H}]^{-}$; HRMS (ESI) $m / z(+)$ : calcd. for $\mathrm{C}_{65} \mathrm{H}_{97} \mathrm{~N}_{12} \mathrm{O}_{19}$ 1349.6987, found 1349.6962; calcd. for $\mathrm{C}_{65} \mathrm{H}_{96} \mathrm{~N}_{12} \mathrm{NaO}_{19}$ 1371.6807, found 1371.6804; calcd. for $\mathrm{C}_{65} \mathrm{H}_{97} \mathrm{~N}_{12} \mathrm{NaO}_{19}$ 686.3440, found 686.3431; calcd. for $\mathrm{C}_{65} \mathrm{H}_{96} \mathrm{~N}_{12} \mathrm{Na}_{2} \mathrm{O}_{19}$ 697.3350, found 697.3342.

\section{Cyclic lipodepsipeptide $\quad \mathrm{C}_{7} \mathrm{H}_{15} \mathrm{CO}-G l u-D-O r n-T y r(\&)-D-T h r-G l u-D-A l a-P r o-G l n-D-T y r-I l e-\&$}

(BPC842). Starting from $\mathrm{C}_{7} \mathrm{H}_{15} \mathrm{CO}-\mathrm{Glu}\left(\mathrm{O}^{t} \mathrm{Bu}\right)-\mathrm{D}-\mathrm{Orn}(\mathrm{Boc})-\mathrm{Tyr}(\mathrm{O}-\mathrm{Ile}-\mathrm{H})-\mathrm{D}-\mathrm{Thr}\left({ }^{t} \mathrm{Bu}\right)-\mathrm{Glu}\left(\mathrm{O}^{t} \mathrm{Bu}\right)-\mathrm{D}-$ Ala-Pro-Gln(Tr)-D-Tyr(Wang)-OH, on-resin cyclization followed by acidolytic cleavage afforded the cyclic lipodepsipeptide BPC842 in 26\% purity. HPLC $(\lambda=220 \mathrm{~nm}) t_{R}=7.13 \mathrm{~min}$; MS $(\mathrm{ESI}) \mathrm{m} / \mathrm{z}(+)$ : 1335.6 $[\mathrm{M}+\mathrm{H}]^{+}$; MS (ESI) $m / z(-)$ : $1333.6[\mathrm{M}-\mathrm{H}]^{-}$. The crude peptide was purified by RP-HPLC obtaining BPC842 in $>99 \%$ purity. $\operatorname{HPLC}(\lambda=220 \mathrm{~nm}) t_{R}=7.26 \mathrm{~min} ;{ }^{1} \mathrm{H}-\mathrm{NMR}\left(400 \mathrm{MHz}, \mathrm{DMSO}-\mathrm{d}_{6}\right.$ $\left.+\mathrm{D}_{2} \mathrm{O}\right) \delta(\mathrm{ppm}): 0.71-0.89(\mathrm{~m}, 12 \mathrm{H}), 1.10-1.22(\mathrm{~m}, 15 \mathrm{H}), 1.43-1.45(\mathrm{~m}, 4 \mathrm{H}), 1.57-1.61(\mathrm{~m}, 2 \mathrm{H})$, 1.71-1.89 (m, 8 H), 1.99-2.22 (m, $10 \mathrm{H}), 2.69-2.76(\mathrm{~m}, 2 \mathrm{H}), 2.84-2.95$ (m, $2 \mathrm{H}), 3.22-3.25(\mathrm{~m}, 1 \mathrm{H})$, 3.50-3.55 (m, $2 \mathrm{H}), 3.98-4.01(\mathrm{~m}, 2 \mathrm{H}), 4.10-4.14(\mathrm{~m}, 1 \mathrm{H}), 4.18-4.24(\mathrm{~m}, 2 \mathrm{H}), 4.38-4.42(\mathrm{~m}, 1 \mathrm{H})$, 4.57-4.61 (m, $1 \mathrm{H}), 4.62-4.69(\mathrm{~m}, 1 \mathrm{H}), 4.81-4.90(\mathrm{~m}, 1 \mathrm{H}), 6.63(\mathrm{~d}, 2 \mathrm{H}, J=8.0 \mathrm{~Hz}), 6.84(\mathrm{~d}, 2 \mathrm{H}, J=$ $8.0 \mathrm{~Hz}), 6.94(\mathrm{~d}, 2 \mathrm{H}, J=8.0 \mathrm{~Hz}), 7.05\left(\mathrm{~d}, 2 \mathrm{H}, J=8.0 \mathrm{~Hz}\right.$ ); MS (ESI) $m / z(+): 668.3[\mathrm{M}+2 \mathrm{H}]^{2+}, 1335.7$ $[\mathrm{M}+\mathrm{H}]^{+}$; MS (ESI) $m / z(-)$ : $1333.6[\mathrm{M}-\mathrm{H}]^{-}$; HRMS (ESI) $m / z(+)$ : calcd. for $\mathrm{C}_{64} \mathrm{H}_{95} \mathrm{~N}_{12} \mathrm{O}_{19} 1335.6831$, 
found 1335.6855; calcd. for $\mathrm{C}_{64} \mathrm{H}_{95} \mathrm{~N}_{12} \mathrm{NaO}_{19}$ 679.3362, found 679.3379; calcd. for $\mathrm{C}_{64} \mathrm{H}_{94} \mathrm{~N}_{12} \mathrm{Na}_{2} \mathrm{O}_{19}$ 690.3271, found 690.3301 .

\section{Cyclic lipodepsipeptide $\quad \mathrm{C}_{7} \mathrm{H}_{15} \mathrm{CO}$-Glu-D-Orn-Tyr(\&)-D-Ser-Glu-D-Ala-Pro-GIn-D-Tyr-Ile-\&} (BPC844). Starting from $\mathrm{C}_{7} \mathrm{H}_{15} \mathrm{CO}-\mathrm{Glu}\left(\mathrm{O}^{t} \mathrm{Bu}\right)$-D-Orn(Boc)-Tyr(O-Ile-H)-D-Ser( $\left.{ }^{t} \mathrm{Bu}\right)-\mathrm{Glu}\left(\mathrm{O}^{t} \mathrm{Bu}\right)-\mathrm{D}-\mathrm{Ala}-$ Pro-Gln(Tr)-D-Tyr(Wang)-OH, on-resin cyclization followed by acidolytic cleavage afforded the cyclic lipodepsipeptide BPC844 in $21 \%$ purity. $\operatorname{HPLC}(\lambda=220 \mathrm{~nm}) t_{R}=7.13 \mathrm{~min} ;{ }^{1} \mathrm{H}-\mathrm{NMR}(400 \mathrm{MHz}$, DMSO-d $\left.{ }_{6}+\mathrm{D}_{2} \mathrm{O}\right) \delta(\mathrm{ppm}):$ 0.73-0.76 (m, $\left.6 \mathrm{H}\right), 0.83-0.85(\mathrm{~m}, 3 \mathrm{H}), 1.10-1.20(\mathrm{~m}, 15 \mathrm{H}), 1.40-1.45(\mathrm{~m}$, $4 \mathrm{H}), 1.56-1.62(\mathrm{~m}, 3 \mathrm{H}), 1.73-1.88(\mathrm{~m}, 8 \mathrm{H}), 2.07-2.24(\mathrm{~m}, 10 \mathrm{H}), 2.71-2.74(\mathrm{~m}, 2 \mathrm{H}), 2.87-2.91$ (m, 2 H), 3.19-3.33 (m, 2 H), 3.47-3.53 (m, 2 H), 3.95-3.97 (m, 1 H), 4.11-4.24 (m, 5 H), 4.36-4.40 (m, 1 H), 4.56-4.62 (m, $2 \mathrm{H}), 4.86-4.87(\mathrm{~m}, 1 \mathrm{H}), 6.63(\mathrm{~d}, 2 \mathrm{H}, J=8.0 \mathrm{~Hz}), 6.86(\mathrm{~d}, 2 \mathrm{H}, J=8.0 \mathrm{~Hz}), 6.94(\mathrm{~d}, 2$ $\mathrm{H}, J=8.0 \mathrm{~Hz}), 7.06(\mathrm{~d}, 2 \mathrm{H}, J=8.0 \mathrm{~Hz})$; MS (ESI) $m / z(+): 1321.6[\mathrm{M}+\mathrm{H}]^{+}$; MS (ESI) $m / z(-): 1319.6$ [M-H] $]^{-}$. The crude peptide was purified by RP-HPLC obtaining BPC844 in 94\% purity. HPLC $(\lambda=$ $220 \mathrm{~nm}) t_{R}=7.12 \mathrm{~min} ; \mathrm{MS}(\mathrm{ESI}) \mathrm{m} / z(+): 661.8[\mathrm{M}+2 \mathrm{H}]^{2+}, 1321.7[\mathrm{M}+\mathrm{H}]^{+} ;$HRMS (ESI) $m / z(+)$ : calcd. for $\mathrm{C}_{63} \mathrm{H}_{93} \mathrm{~N}_{12} \mathrm{O}_{19}$ 1321.6674, found 1321.6701; calcd. for $\mathrm{C}_{63} \mathrm{H}_{92} \mathrm{~N}_{12} \mathrm{NaO}_{19}$ 1343.6494, found 1343.6521.

\section{Cyclic lipodepsipeptide $\quad \mathrm{C}_{7} \mathrm{H}_{15} \mathrm{CO}-G l u-D-O r n-D-T y r(\&)-D-T h r-G l u-D-V a l-P r o-G l n-T y r-I l e-\&$} (BPC846). Starting from the peptidyl resin $\mathrm{C}_{7} \mathrm{H}_{15} \mathrm{CO}-\mathrm{Glu}\left(\mathrm{O}^{t} \mathrm{Bu}\right)-\mathrm{D}-\mathrm{Orn}(\mathrm{Boc})-\mathrm{D}-\mathrm{Tyr}(\mathrm{O}-\mathrm{Ile}-\mathrm{H})-\mathrm{D}-$ $\operatorname{Thr}\left({ }^{t} \mathrm{Bu}\right)-\mathrm{Glu}\left(\mathrm{O}^{t} \mathrm{Bu}\right)-\mathrm{D}-\mathrm{Val}-\mathrm{Pro}-\mathrm{G} \ln (\mathrm{Tr})-\mathrm{Tyr}(\mathrm{Wang})-\mathrm{OH}$, on-resin cyclization followed by acidolytic cleavage afforded a mixture of the cyclic lipodepsipeptide BPC846 (17\% purity, $\left.t_{R}=7.19 \mathrm{~min}\right)$ and the linear product from the hydrolysis of the macrolactone $\mathrm{C}_{7} \mathrm{H}_{15} \mathrm{CO}-\mathrm{Glu}-\mathrm{D}-\mathrm{Orn}-\mathrm{D}-\mathrm{Tyr}-\mathrm{D}-\mathrm{Thr}-\mathrm{Glu}-\mathrm{D}-\mathrm{Val}$ Pro-Gln-Tyr-Ile-OH (17\% purity, $\left.t_{R}=7.04 \mathrm{~min}\right)$ [1:1 ratio by MS (ESI)]. MS (ESI) $m / z(+): 1363.7$ $[\mathrm{M}+\mathrm{H}]^{+}, 1381.7\left[\mathrm{M}+\mathrm{H}_{2} \mathrm{O}+\mathrm{H}\right]^{+}, 1403.7\left[\mathrm{M}+\mathrm{H}_{2} \mathrm{O}+\mathrm{Na}\right]^{+}$; $\mathrm{MS}$ (ESI) $m / z(-): 1361.7[\mathrm{M}-\mathrm{H}]^{-}, 1379.6$ $\left[\mathrm{M}+\mathrm{H}_{2} \mathrm{O}-\mathrm{H}\right]^{-}, 1402.6\left[\mathrm{M}+\mathrm{H}_{2} \mathrm{O}+\mathrm{Na}-2 \mathrm{H}\right]^{-}$. The crude mixture was purified by RP-HPLC obtaining 
BPC846 (59\% purity, $\left.t_{R}=7.11 \mathrm{~min}\right)$ together with the linear product from the hydrolysis of the macrolactone (37\% purity, $t_{R}=6.97 \mathrm{~min}$ ). MS (ESI) $\mathrm{m} / z(+): 682.3[\mathrm{M}+2 \mathrm{H}]^{2+}, 691.3\left[\mathrm{M}+\mathrm{H}_{2} \mathrm{O}+2 \mathrm{H}\right]^{2+}$, $1363.7[\mathrm{M}+\mathrm{H}]^{+}, 1381.7\left[\mathrm{M}+\mathrm{H}_{2} \mathrm{O}+\mathrm{H}\right]^{+}$; MS (ESI) $m / z(-): 1361.6[\mathrm{M}-\mathrm{H}]^{-}, 1379.6\left[\mathrm{M}+\mathrm{H}_{2} \mathrm{O}-\mathrm{H}\right]^{-} ; \mathrm{HRMS}$ (ESI) $m / z(+)$ : calcd. for $\mathrm{C}_{66} \mathrm{H}_{99} \mathrm{~N}_{12} \mathrm{O}_{19}$ 1363.7144, found 1363.7118; calcd for $\mathrm{C}_{66} \mathrm{H}_{98} \mathrm{~N}_{12} \mathrm{NaO}_{19}$ 1385.6963, found 1385.6984; calcd for $\mathrm{C}_{66} \mathrm{H}_{101} \mathrm{~N}_{12} \mathrm{O}_{20}$ 1381.7250, found 1381.7242; calcd for $\mathrm{C}_{66} \mathrm{H}_{100} \mathrm{~N}_{12} \mathrm{NaO}_{20}$ 1403.7069, found 1403.7063.

\section{Cyclic lipodepsipeptide $\quad \mathrm{C}_{7} \mathrm{H}_{15} \mathrm{CO}-G$ lu-D-Orn-D-Tyr(\&)-D-Ser-Glu-D-Val-Pro-Gln-Tyr-Ile-\&}

(BPC848). Starting from the peptidyl resin $\mathrm{C}_{7} \mathrm{H}_{15} \mathrm{CO}-\mathrm{Glu}\left(\mathrm{O}^{t} \mathrm{Bu}\right)-\mathrm{D}-\mathrm{Orn}(\mathrm{Boc})-\mathrm{D}-\mathrm{Ty}(\mathrm{O}-\mathrm{Ile}-\mathrm{H})-\mathrm{D}-$ $\operatorname{Ser}\left({ }^{t} \mathrm{Bu}\right)-\mathrm{Glu}\left(\mathrm{O}^{t} \mathrm{Bu}\right)-\mathrm{D}-\mathrm{Val}-\mathrm{Pro}-\mathrm{G} \ln (\mathrm{Tr})-\mathrm{Tyr}(\mathrm{Wang})-\mathrm{OH}$, on-resin cyclization followed by acidolytic cleavage afforded a mixture of the cyclic lipodepsipeptide BPC848 (10\% purity, $\left.t_{R}=7.18 \mathrm{~min}\right)$ and the linear product from the hydrolysis of the macrolactone $\mathrm{C}_{7} \mathrm{H}_{15} \mathrm{CO}-\mathrm{Glu}-\mathrm{D}-\mathrm{Orn}-\mathrm{D}-\mathrm{Tyr}-\mathrm{D}-\mathrm{Ser}-\mathrm{Glu}-\mathrm{D}-\mathrm{Val}-$ Pro-Gln-Tyr-Ile-OH (17\% purity, $\left.t_{R}=7.00 \mathrm{~min}\right)$ [3:4 ratio by MS (ESI)]. MS (ESI) $\mathrm{m} / z(+): 685.4$ $\left[\mathrm{M}+\mathrm{H}_{2} \mathrm{O}+2 \mathrm{H}\right]^{2+}, 1350.7[\mathrm{M}+\mathrm{H}]^{+}, 1367.7\left[\mathrm{M}+\mathrm{H}_{2} \mathrm{O}+\mathrm{H}\right]^{+}$; MS (ESI) $m / z(-): 682.2\left[\mathrm{M}+\mathrm{H}_{2} \mathrm{O}-2 \mathrm{H}\right]^{2-}, 1348.6$ [M-H]', $1366.7\left[\mathrm{M}+\mathrm{H}_{2} \mathrm{O}-\mathrm{H}\right]^{-}, 1387.6\left[\mathrm{M}+\mathrm{H}_{2} \mathrm{O}+\mathrm{Na}-2 \mathrm{H}\right]^{-}$. The crude mixture was purified by RP-HPLC obtaining BPC848 (38\% purity, $\left.t_{R}=7.09 \mathrm{~min}\right)$ together with the linear product from the hydrolysis of the macrolactone $\left(62 \%\right.$ purity, $\left.t_{R}=6.93 \mathrm{~min}\right) . \mathrm{MS}(\mathrm{ESI}) \mathrm{m} / \mathrm{z}(+): 675.3[\mathrm{M}+2 \mathrm{H}]^{2+}, 1349.7[\mathrm{M}+\mathrm{H}]^{+}$, $1367.7\left[\mathrm{M}+\mathrm{H}_{2} \mathrm{O}+\mathrm{H}\right]^{+}$; MS (ESI) $m / z(-): 1347.6[\mathrm{M}-\mathrm{H}]^{-}, 1365.6\left[\mathrm{M}+\mathrm{H}_{2} \mathrm{O}-\mathrm{H}\right]^{-} ; \mathrm{HRMS}(\mathrm{ESI}) m / z(+)$ : calcd. for $\mathrm{C}_{65} \mathrm{H}_{97} \mathrm{~N}_{12} \mathrm{O}_{19}$ 1349.6987, found 1349.6999; calcd. for $\mathrm{C}_{65} \mathrm{H}_{96} \mathrm{~N}_{12} \mathrm{NaO}_{19}$ 1371.6807, found 1371.6827; calcd. for $\mathrm{C}_{65} \mathrm{H}_{99} \mathrm{~N}_{12} \mathrm{O}_{20}$ 1367.7093, found 1367.7079 ; calcd. for $\mathrm{C}_{65} \mathrm{H}_{98} \mathrm{~N}_{12} \mathrm{NaO}_{20}$ 1389.6913, found 1389.6902 .

\section{Cyclic lipodepsipeptide $\quad \mathrm{C}_{7} \mathrm{H}_{15} \mathrm{CO}-G l u-D-O r n-D-T y r(\&)-D-T h r-G l u-D-A l a-P r o-G l n-T y r-I l e-\&$}

(BPC850). Starting from the peptidyl resin $\mathrm{C}_{7} \mathrm{H}_{15} \mathrm{CO}-\mathrm{Glu}\left(\mathrm{O}^{t} \mathrm{Bu}\right)-\mathrm{D}-\mathrm{Orn}(\mathrm{Boc})-\mathrm{D}-\mathrm{Tyr}(\mathrm{O}-\mathrm{Ile}-\mathrm{H})-\mathrm{D}-$ $\operatorname{Thr}\left({ }^{t} \mathrm{Bu}\right)-\mathrm{Glu}\left(\mathrm{O}^{t} \mathrm{Bu}\right)$-D-Ala-Pro-Gln(Tr)-Tyr(Wang)-OH, on-resin cyclization followed by acidolytic 
cleavage afforded a mixture of the cyclic lipodepsipeptide BPC850 (14\% purity, $\left.t_{R}=7.07 \mathrm{~min}\right)$ and the linear product from the hydrolysis of the macrolactone $\mathrm{C}_{7} \mathrm{H}_{15} \mathrm{CO}-\mathrm{Glu}-\mathrm{D}-\mathrm{Orn}-\mathrm{D}-\mathrm{Tyr}-\mathrm{D}-\mathrm{Thr}-\mathrm{Glu}-\mathrm{D}-\mathrm{Ala}-$ Pro-Gln-Tyr-Ile-OH (16\% purity, $\left.t_{R}=6.93 \mathrm{~min}\right)[3: 4$ ratio by MS (ESI)]. MS (ESI) $\mathrm{m} / z(+): 677.3$ $\left[\mathrm{M}+\mathrm{H}_{2} \mathrm{O}+2 \mathrm{H}\right]^{2+}, 1335.7[\mathrm{M}+\mathrm{H}]^{+}, 1353.7\left[\mathrm{M}+\mathrm{H}_{2} \mathrm{O}+\mathrm{H}\right]^{+}, 1375.7\left[\mathrm{M}+\mathrm{H}_{2} \mathrm{O}+\mathrm{Na}\right]^{+} ; \mathrm{MS}(\mathrm{ESI}) \mathrm{m} / z(-): 675.1$ $\left[\mathrm{M}+\mathrm{H}_{2} \mathrm{O}-2 \mathrm{H}\right]^{2-}, 1333.6[\mathrm{M}-\mathrm{H}]^{-}, 1351.6\left[\mathrm{M}+\mathrm{H}_{2} \mathrm{O}-\mathrm{H}\right]^{-}, 1373.6\left[\mathrm{M}+\mathrm{H}_{2} \mathrm{O}+\mathrm{Na}-2 \mathrm{H}\right]^{-}$. The crude mixture was purified by RP-HPLC obtaining BPC850 (48\% purity, $t_{R}=6.96 \mathrm{~min}$ ) together with the linear product from the hydrolysis of the macrolactone ( $52 \%$ purity, $t_{R}=6.84 \mathrm{~min}$ ). MS (ESI) $\mathrm{m} / z(+): 668.8$ $[\mathrm{M}+2 \mathrm{H}]^{2+}, 1335.7[\mathrm{M}+\mathrm{H}]^{+}, 1353.6\left[\mathrm{M}+\mathrm{H}_{2} \mathrm{O}+\mathrm{H}\right]^{+} ; \mathrm{MS}(\mathrm{ESI}) m / z(-): 1333.6[\mathrm{M}-\mathrm{H}]^{-}, 1351.6\left[\mathrm{M}+\mathrm{H}_{2} \mathrm{O}-\right.$ $\mathrm{H}]^{-}$; HRMS (ESI) $m / z$ (+): calcd for $\mathrm{C}_{64} \mathrm{H}_{95} \mathrm{~N}_{12} \mathrm{O}_{19}$ 1335.6831, found 1335.6812; calcd for $\mathrm{C}_{64} \mathrm{H}_{94} \mathrm{~N}_{12} \mathrm{NaO}_{19}$ 1357.6650, found 1357.6648; calcd for $\mathrm{C}_{64} \mathrm{H}_{97} \mathrm{~N}_{12} \mathrm{O}_{20}$ 1353.6937, found 1353.6909; calcd for $\mathrm{C}_{64} \mathrm{H}_{96} \mathrm{~N}_{12} \mathrm{NaO}_{20}$ 1375.6756, found 1375.6727.

\section{Cyclic lipodepsipeptide $\quad \mathrm{C}_{7} \mathrm{H}_{15} \mathrm{CO}$-Glu-D-Orn-D-Tyr(\&)-D-Ser-Glu-D-Ala-Pro-Gln-Tyr-Ile-\&}

(BPC852). Starting from the peptidyl resin $\mathrm{C}_{7} \mathrm{H}_{15} \mathrm{CO}-\mathrm{Glu}\left(\mathrm{O}^{t} \mathrm{Bu}\right)-\mathrm{D}-\mathrm{Orn}(\mathrm{Boc})-\mathrm{D}-\mathrm{Ty}(\mathrm{O}-\mathrm{Ile}-\mathrm{H})-\mathrm{D}-$ $\operatorname{Ser}\left({ }^{t} \mathrm{Bu}\right)-\mathrm{Glu}\left(\mathrm{O}^{t} \mathrm{Bu}\right)-\mathrm{D}-\mathrm{Ala}-\mathrm{Pro}-\mathrm{G} \ln (\mathrm{Tr})-\mathrm{Tyr}(\mathrm{Wang})-\mathrm{OH}$, on-resin cyclization followed by acidolytic cleavage afforded a mixture of the cyclic lipodepsipeptide BPC852 (11\% purity, $\left.t_{R}=7.06 \mathrm{~min}\right)$ and the linear product from the hydrolysis of the macrolactone $\mathrm{C}_{7} \mathrm{H}_{15} \mathrm{CO}-\mathrm{Glu}-\mathrm{D}-\mathrm{Orn}-\mathrm{D}-\mathrm{Tyr}-\mathrm{D}-\mathrm{Ser}-\mathrm{Glu}-\mathrm{D}-\mathrm{Ala}$ Pro-Gln-Tyr-Ile-OH (18\% purity, $\left.t_{R}=6.88 \mathrm{~min}\right)$ [1:2 ratio by MS (ESI)]. MS (ESI) $\mathrm{m} / z(+): 1321.7$ $[\mathrm{M}+\mathrm{H}]^{+}, 1339.7\left[\mathrm{M}+\mathrm{H}_{2} \mathrm{O}+\mathrm{H}\right]^{+} ; \mathrm{MS} \quad(\mathrm{ESI}) \quad m / z(-): 1319.6[\mathrm{M}-\mathrm{H}]^{-}, 1337.6\left[\mathrm{M}+\mathrm{H}_{2} \mathrm{O}-\mathrm{H}\right]^{-}, 1359.6$ $\left[\mathrm{M}+\mathrm{H}_{2} \mathrm{O}+\mathrm{Na}-2 \mathrm{H}\right]^{-}$. The crude mixture was purified by RP-HPLC obtaining BPC852 $\left(81 \%\right.$ purity, $t_{R}=$ $6.95 \mathrm{~min})$ together with the linear product from the hydrolysis of the macrolactone $\left(19 \%\right.$ purity, $t_{R}=$ $6.78 \mathrm{~min}) . \mathrm{MS}(\mathrm{ESI}) \mathrm{m} / z(+): 1321.6[\mathrm{M}+\mathrm{H}]^{+}, 1339.6\left[\mathrm{M}+\mathrm{H}_{2} \mathrm{O}+\mathrm{H}\right]^{+}$; MS (ESI) $m / z(-): 1319.6[\mathrm{M}-\mathrm{H}]^{-}$, $1337.6\left[\mathrm{M}+\mathrm{H}_{2} \mathrm{O}-\mathrm{H}\right]^{-}$; HRMS (ESI) $\mathrm{m} / z(+)$ : calcd. for $\mathrm{C}_{63} \mathrm{H}_{93} \mathrm{~N}_{12} \mathrm{O}_{19}$ 1321.6674, found 1321.6653; 
calcd. for $\mathrm{C}_{63} \mathrm{H}_{92} \mathrm{~N}_{12} \mathrm{NaO}_{19}$ 1343.6494, found 1343.6510; calcd. for $\mathrm{C}_{63} \mathrm{H}_{95} \mathrm{~N}_{12} \mathrm{O}_{20}$ 1339.6780, found 1339.6800; calcd. for $\mathrm{C}_{63} \mathrm{H}_{94} \mathrm{~N}_{12} \mathrm{NaO}_{20} 1361.6600$, found 1361.6581 .

\section{Cyclic lipodepsipeptide $\quad \mathrm{C}_{11} \mathrm{H}_{23} \mathrm{CO}$-Glu-D-Orn-Tyr(\&)-D-Thr-Glu-D-Val-Pro-Gln-D-Tyr-Ile-\&}

(BPC854). Starting from 8b, on-resin cyclization followed by acidolytic cleavage afforded the cyclic lipodepsipeptide BPC854 in 49\% purity. $\operatorname{HPLC}(\lambda=220 \mathrm{~nm}) t_{R}=8.21 \mathrm{~min}$. MS (ESI) $\mathrm{m} / z(+): 1420.8$ $[\mathrm{M}+\mathrm{H}]^{+}$; MS (ESI) $m / z(-): 1417.8[\mathrm{M}-\mathrm{H}]^{-}$. The crude peptide was purified by RP-HPLC obtaining BPC854 in 92\% purity. HPLC $(\lambda=220 \mathrm{~nm}) t_{R}=7.99 \mathrm{~min} ;{ }^{1} \mathrm{H}-\mathrm{NMR}\left(400 \mathrm{MHz}, \mathrm{DMSO}-\mathrm{d}_{6}+\mathrm{D}_{2} \mathrm{O}\right) \delta$ (ppm): 0.71-0.85 (m, $18 \mathrm{H}), 1.14-1.19(\mathrm{~m}, 18 \mathrm{H}), 1.40-1.58(\mathrm{~m}, 6 \mathrm{H}), 1.61-1.74(\mathrm{~m}, 4 \mathrm{H}), 1.87-2.25$ (m, $14 \mathrm{H}), 2.65-2.76$ (m, $3 \mathrm{H}), 2.80-2.85$ (m, $2 \mathrm{H}), 3.02-3.21$ (m, $2 \mathrm{H}), 3.26-3.34(\mathrm{~m}, 1 \mathrm{H}), 3.48-3.50$ (m, 1 H), 3.80-3.87 (m, 2 H), 3.96-4.02 (m, 2 H), 4.11-4.25 (m, 4 H), 4.42-4.46 (m, 1 H), 4.56-4.60 (m, 2 H), $6.61(\mathrm{~d}, 2 \mathrm{H}, J=8.0 \mathrm{~Hz}), 6.83(\mathrm{~d}, 2 \mathrm{H}, J=8.0 \mathrm{~Hz}), 6.99-7.03(\mathrm{~m}, 4 \mathrm{H})$; MS (ESI) $m / z(+): 1419.8$ $[\mathrm{M}+\mathrm{H}]^{+}$; MS (ESI) $m / z(-): 1417.7$ [M-H] ; HRMS (ESI) $m / z(+)$ : calcd. for $\mathrm{C}_{70} \mathrm{H}_{107} \mathrm{~N}_{12} \mathrm{O}_{19} 1419.7770$, found 1419.7763; calcd. for $\mathrm{C}_{70} \mathrm{H}_{106} \mathrm{~N}_{12} \mathrm{NaO}_{19} 1441.7589$, found 1441.7593 .

\section{Cyclic lipodepsipeptide $\quad \mathrm{C}_{15} \mathrm{H}_{31} \mathrm{CO}-G l u-D-O r n-T y r(\&)-D-T h r-G l u-D-V a l-P r o-G I n-D-T y r-I l e-\&$}

(BPC856). Starting from 8c, on-resin cyclization followed by acidolytic cleavage afforded the cyclic lipodepsipeptide BPC856 in 50\% purity. $\operatorname{HPLC}(\lambda=220 \mathrm{~nm}) t_{R}=9.25 \mathrm{~min}$. MS (ESI) $\mathrm{m} / z(+): 1475.8$ $[\mathrm{M}+\mathrm{H}]^{+}, 1497.8[\mathrm{M}+\mathrm{Na}]^{+}$; MS (ESI) $m / z(-): 1473.8[\mathrm{M}-\mathrm{H}]^{-}$. The crude peptide was purified by RPHPLC yielding BPC856 in 92\% purity. $\operatorname{HPLC}(\lambda=220 \mathrm{~nm}) t_{R}=9.23 \mathrm{~min} ;{ }^{1} \mathrm{H}-\mathrm{NMR}(400 \mathrm{MHz}$, DMSO- $\left.\mathrm{d}_{6}+\mathrm{D}_{2} \mathrm{O}\right) \delta(\mathrm{ppm}): 0.79-0.89(\mathrm{~m}, 18 \mathrm{H}), 1.14-1.22(\mathrm{~m}, 28 \mathrm{H}), 1.40-1.50(\mathrm{~m}, 6 \mathrm{H}), 1.56-1.63$ (m, 2 H), 1.69-1.77 (m, 2 H), 1.84-2.33 (m, 14 H), 2.68-2.73 (m, 4 H), 2.88-2.90 (m, 2 H), 3.26-3.37 (m, 2 H), 3.60-3.63 (m, 1 H), 4.00-4.04 (m, 2 H), 4.11-4.17 (m, 2 H), 4.23-4.28 (m, 2 H), 4.43-4.46 (m, $1 \mathrm{H}), 4.48-4.51(\mathrm{~m}, 1 \mathrm{H}), 4.56-4.60(\mathrm{~m}, 1 \mathrm{H}), 4.70-4.73(\mathrm{~m}, 1 \mathrm{H}), 6.63(\mathrm{~d}, 2 \mathrm{H}, J=8.0 \mathrm{~Hz}), 6.83(\mathrm{~d}, 2$ $\mathrm{H}, J=8.0 \mathrm{~Hz}), 6.93(\mathrm{~d}, 2 \mathrm{H}, J=8.0 \mathrm{~Hz}), 7.04(\mathrm{~d}, 2 \mathrm{H}, J=8.0 \mathrm{~Hz})$; MS (ESI) $m / z(+): 738.4[\mathrm{M}+2 \mathrm{H}]^{2+}$, 
$749.9[\mathrm{M}+\mathrm{Na}+\mathrm{H}]^{2+}, 1475.9[\mathrm{M}+\mathrm{H}]^{+}, 1497.9[\mathrm{M}+\mathrm{Na}]^{+} ; \mathrm{MS}(\mathrm{ESI}) \mathrm{m} / z(-)$ : $1473.9[\mathrm{M}-\mathrm{H}]^{-}, 1495.8$ [M+Na-2H]; HRMS (ESI) $m / z(+)$ : calcd. for $\mathrm{C}_{74} \mathrm{H}_{115} \mathrm{~N}_{12} \mathrm{O}_{19}$ 1475.8396, found 1475.8376; calcd. for $\mathrm{C}_{74} \mathrm{H}_{114} \mathrm{~N}_{12} \mathrm{NaO}_{19}$ 1497.8215, found 1497.8227.

\section{Cyclic lipodepsipeptide $\mathrm{C}_{11} \mathrm{H}_{23} \mathrm{CO}-G l u-D-O r n-D-T y r(\&)-D-T h r-G l u-D-V a l-P r o-G I n-T y r-I l e-\&$}

(BPC858). Starting from the peptidyl resin $\mathrm{C}_{11} \mathrm{H}_{23} \mathrm{CO}-\mathrm{Glu}\left(\mathrm{O}^{t} \mathrm{Bu}\right)-\mathrm{D}-\mathrm{Orn}(\mathrm{Boc})-\mathrm{D}-\mathrm{Tyr}(\mathrm{O}-\mathrm{Ile}-\mathrm{H})-\mathrm{D}-$ $\operatorname{Thr}\left({ }^{t} \mathrm{Bu}\right)-\mathrm{Glu}\left(\mathrm{O}^{t} \mathrm{Bu}\right)-\mathrm{D}-$ Val-Pro-Gln(Tr)-Tyr(Wang)-OH, on-resin cyclization followed by acidolytic cleavage afforded a mixture of the cyclic lipodepsipeptide BPC858 $\left(16 \%\right.$ purity, $\left.t_{R}=8.39 \mathrm{~min}\right)$ and the linear product from the hydrolysis of the macrolactone $\mathrm{C}_{11} \mathrm{H}_{23} \mathrm{CO}-\mathrm{Glu}-\mathrm{D}-\mathrm{Orn}-\mathrm{D}-\mathrm{Tyr}-\mathrm{D}-\mathrm{Thr}-\mathrm{Glu}-\mathrm{D}-\mathrm{Val}$ Pro-Gln-Tyr-Ile-OH (21\% purity, $\left.t_{R}=8.23 \mathrm{~min}\right)[2.5: 4$ ratio by MS (ESI)]. MS (ESI) $m / z(+): 1419.9$ $[\mathrm{M}+\mathrm{H}]^{+}, 1437.9\left[\mathrm{M}+\mathrm{H}_{2} \mathrm{O}+\mathrm{H}\right]^{+}, 1459.8\left[\mathrm{M}+\mathrm{H}_{2} \mathrm{O}+\mathrm{Na}\right]^{+}$; $\mathrm{MS}$ (ESI) $m / z(-): 1418.8[\mathrm{M}-\mathrm{H}]^{-}, 1435.8$ $\left[\mathrm{M}+\mathrm{H}_{2} \mathrm{O}-\mathrm{H}\right]^{-}, \quad 1457.8 \quad\left[\mathrm{M}+\mathrm{H}_{2} \mathrm{O}+\mathrm{Na}-2 \mathrm{H}\right]^{-}$. The mixture was purified by reverse-phase column chromatography. The cyclic lipodepsipeptide BPC858 was obtained in 78\% purity. HPLC $(\lambda=220$ nm) $t_{R}=8.14 \mathrm{~min} ; \mathrm{MS}(\mathrm{ESI}) \mathrm{m} / z(+): 1419.9[\mathrm{M}+\mathrm{H}]^{+}, 1441.9[\mathrm{M}+\mathrm{Na}]^{+}$; MS (ESI) $m / z(-): 1417.8[\mathrm{M}-$ $\mathrm{H}]^{-}, \quad 1439.7 \quad[\mathrm{M}+\mathrm{Na}-2 \mathrm{H}]^{-} . \quad \mathrm{C}_{11} \mathrm{H}_{23} \mathrm{CO}-\mathrm{Glu}-\mathrm{D}-\mathrm{Orn}-\mathrm{D}-\mathrm{Tyr}-\mathrm{D}-\mathrm{Th}$-Glu-D-Val-Pro-Gln-Tyr-Ile-OH was obtained in $91 \%$ purity. HPLC $(\lambda=220 \mathrm{~nm}) t_{R}=7.99 \mathrm{~min}$; MS (ESI) $\mathrm{m} / \mathrm{z}(+): 1437.9[\mathrm{M}+\mathrm{H}]^{+}, 1459.8$ $[\mathrm{M}+\mathrm{Na}]^{+}$; MS (ESI) $m / z(-): 1435.8[\mathrm{M}-\mathrm{H}]^{-}, 1457.8[\mathrm{M}+\mathrm{Na}-2 \mathrm{H}]^{-}$.

\section{Cyclic lipodepsipeptide $\quad \mathrm{C}_{15} \mathrm{H}_{31} \mathrm{CO}-G l u-D-O r n-D-T y r(\&)-D-T h r-G l u-D-V a l-P r o-G I n-T y r-I l e-\&$} (BPC860). Starting from the peptidyl resin $\mathrm{C}_{15} \mathrm{H}_{31} \mathrm{CO}-\mathrm{Glu}\left(\mathrm{O}^{t} \mathrm{Bu}\right)-\mathrm{D}-\mathrm{Orn}(\mathrm{Boc})-\mathrm{D}-\mathrm{Tyr}(\mathrm{O}-\mathrm{Ile}-\mathrm{H})-\mathrm{D}-$ $\operatorname{Thr}\left({ }^{t} \mathrm{Bu}\right)-\mathrm{Glu}\left(\mathrm{O}^{t} \mathrm{Bu}\right)-\mathrm{D}-\mathrm{Val}-\mathrm{Pro}-\mathrm{G} \ln (\mathrm{Tr})-\mathrm{Tyr}(\mathrm{Wang})-\mathrm{OH}$, on-resin cyclization followed by acidolytic cleavage afforded a mixture of the cyclic lipodepsipeptide BPC860 (24\% purity, $\left.t_{R}=9.04 \mathrm{~min}\right)$ and the linear product from the hydrolysis of the macrolactone $\mathrm{C}_{15} \mathrm{H}_{31} \mathrm{CO}-\mathrm{Glu}-\mathrm{D}-\mathrm{Orn}-\mathrm{D}-\mathrm{Tyr}-\mathrm{D}-\mathrm{Thr}-\mathrm{Glu}-\mathrm{D}-\mathrm{Val}-$ Pro-Gln-Tyr-Ile-OH (25\% purity, $\left.t_{R}=8.88 \mathrm{~min}\right)$ [2:3 ratio by MS (ESI)]. MS (ESI) $m / z(+): 1475.8$ $[\mathrm{M}+\mathrm{H}]^{+}, 1493.8\left[\mathrm{M}+\mathrm{H}_{2} \mathrm{O}+\mathrm{H}\right]^{+}, 1515.8\left[\mathrm{M}+\mathrm{H}_{2} \mathrm{O}+\mathrm{Na}\right]^{+}$; $\mathrm{MS}$ (ESI) $m / z(-): 1473.7[\mathrm{M}-\mathrm{H}]^{-}, 1491.7$ 
$\left[\mathrm{M}+\mathrm{H}_{2} \mathrm{O}-\mathrm{H}\right]^{-}, \quad 1514.7 \quad[\mathrm{M}+\mathrm{Na}-2 \mathrm{H}]^{-}$. The mixture was purified by reverse-phase column chromatography. Cyclic lipodepsipeptide BPC860 was obtained in 70\% purity. HPLC $(\lambda=220 \mathrm{~nm}) t_{R}$ $=9.06 \mathrm{~min} ; \mathrm{MS}(\mathrm{ESI}) \mathrm{m} / z(+): 1476.0[\mathrm{M}+\mathrm{H}]^{+}, 1497.9[\mathrm{M}+\mathrm{Na}]^{+}$; MS (ESI) $m / z(-): 1473.9[\mathrm{M}-\mathrm{H}]^{-}$, 1495.8 [M+Na-2H]'. $\mathrm{C}_{15} \mathrm{H}_{31} \mathrm{CO}-\mathrm{Mlu}-\mathrm{D}-\mathrm{Orn}-\mathrm{D}-\mathrm{Tyr}-\mathrm{D}-\mathrm{Thr}-\mathrm{Glu}-\mathrm{D}-\mathrm{Val}-\mathrm{Pro}-\mathrm{Gln}-\mathrm{Tyr}-\mathrm{Ile}-\mathrm{OH}$ was obtained in $78 \%$ purity. $\operatorname{HPLC}(\lambda=220 \mathrm{~nm}) t_{R}=8.89 \mathrm{~min}$; MS (ESI) $m / z(+): 1494.0[\mathrm{M}+\mathrm{H}]^{+}, 1516.0[\mathrm{M}+\mathrm{Na}]^{+}$; MS (ESI) $m / z(-): 1491.9[\mathrm{M}-\mathrm{H}]^{-}, 1513.9[\mathrm{M}+\mathrm{Na}-2 \mathrm{H}]^{-}$.

\section{General method for the hydrolysis of cyclic depsipeptides}

Cyclic depsipeptide 4 and cyclic lipodepsipeptides BPC846, BPC848, BPC850, BPC852, BPC854, BPC856, BPC858 and BPC860 were treated with a solution of $\mathrm{CH}_{3} \mathrm{OH} / \mathrm{H}_{2} \mathrm{O} / \mathrm{NH}_{3}(4: 1: 1)$ for $24 \mathrm{~h}$ at room temperature. ${ }^{42}$ Next, the mixture was evaporated and the crude residue was dissolved in $\mathrm{H}_{2} \mathrm{O} / \mathrm{CH}_{3} \mathrm{CN}$, lyophilized, analyzed by HPLC and characterized by mass spectrometry.

pNZ-Tyr-D-Thr-Glu-D-Val-Pro-GIn-D-Tyr-Ile-OH. The hydrolysis of the peptide 4 was performed following the methodology described above. $p$ NZ-Tyr-D-Thr-Glu-D-Val-Pro-Gln-D-Tyr-Ile-OH $\left(t_{R}=\right.$ 7.34 min, 26\% HPLC purity) together with the methyl ester derivative $p$ NZ-Tyr-D-Thr-Glu-D-Val-ProGln-D-Tyr-Ile-OMe $\left(t_{R}=7.49 \mathrm{~min}, 20 \%\right.$ HPLC purity) were obtained; MS (ESI) $\mathrm{m} / z(+): 1191.5$ $[\mathrm{M}+\mathrm{H}]^{+}, 1205.5[\mathrm{M}(\mathrm{OMe})+\mathrm{H}]^{+}, 1213.5[\mathrm{M}+\mathrm{Na}]^{+}, 1227.5[\mathrm{M}(\mathrm{OMe})+\mathrm{Na}]^{+}$; MS (ESI) $m / z(-): 1189.5$ $[\mathrm{M}-\mathrm{H}]^{-}, 1203.5[\mathrm{M}(\mathrm{OMe})-\mathrm{H}]^{-}$ 
$\mathrm{C}_{7} \mathrm{H}_{15} \mathrm{CO}$-Glu-D-Orn-D-Tyr-D-Thr-Glu-D-Val-Pro-GIn-Tyr-Ile-OH. The hydrolysis of the peptide BPC846 was performed following the methodology described above. $\mathrm{C}_{7} \mathrm{H}_{15} \mathrm{CO}-\mathrm{Glu}-\mathrm{D}-\mathrm{Orn}-\mathrm{D}-\mathrm{Tyr}-\mathrm{D}-$ Thr-Glu-D-Val-Pro-Gln-Tyr-Ile-OH $\left(t_{R}=7.04 \mathrm{~min}, 30 \%\right.$ HPLC purity) together with the methyl ester derivative $\mathrm{C}_{7} \mathrm{H}_{15} \mathrm{CO}-\mathrm{Glu}$-D-Orn-D-Tyr-D-Thr-Glu-D-Val-Pro-Gln-Tyr-Ile-OMe $\left(t_{R}=7.24 \mathrm{~min}, 30 \%\right.$ HPLC purity) were obtained; MS (ESI) $m / z(+)$ : $1381.7[\mathrm{M}+\mathrm{H}]^{+}, 1395.7[\mathrm{M}(\mathrm{OMe})+\mathrm{H}]^{+}$; MS (ESI) $m / z(-): 1379.6[\mathrm{M}-\mathrm{H}]^{-}, 1393.7$ [M(OMe)-H]', $1401.6[\mathrm{M}+\mathrm{Na}-2 \mathrm{H}]^{-}, 1415.6[\mathrm{M}(\mathrm{OMe})+\mathrm{Na}-2 \mathrm{H}]^{-}$.

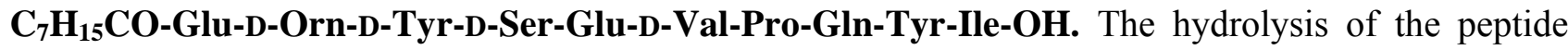
BPC848 was performed following the methodology described above. $\mathrm{C}_{7} \mathrm{H}_{15} \mathrm{CO}-\mathrm{Glu}$-D-Orn-D-Tyr-DSer-Glu-D-Val-Pro-Gln-Tyr-Ile-OH $\left(t_{R}=7.00 \mathrm{~min}, 28 \%\right.$ HPLC purity) together with the methyl ester derivative $\mathrm{C}_{7} \mathrm{H}_{15} \mathrm{CO}-\mathrm{Glu}-\mathrm{D}-\mathrm{Orn}-\mathrm{D}-\mathrm{Tyr}-\mathrm{D}-\mathrm{Ser}-\mathrm{Glu}-\mathrm{D}-\mathrm{Val}-\mathrm{Pro}-\mathrm{Gln}-\mathrm{Tyr}-\mathrm{Ile}-\mathrm{OMe}\left(t_{R}=7.20 \mathrm{~min}, 27 \%\right.$ HPLC purity) were obtained; MS (ESI) $m / z(+): 1367.7[\mathrm{M}+\mathrm{H}]^{+}, 1381.7[\mathrm{M}(\mathrm{OMe})+\mathrm{H}]^{+}, 1389.7$ $[\mathrm{M}+\mathrm{Na}]^{+} ; \mathrm{MS}(\mathrm{ESI}) \mathrm{m} / z(-): 1365.6[\mathrm{M}-\mathrm{H}]^{-}, 1379.7[\mathrm{M}(\mathrm{OMe})-\mathrm{H}]^{-}, 1387.6[\mathrm{M}+\mathrm{Na}-2 \mathrm{H}]^{-}$.

$\mathrm{C}_{7} \mathrm{H}_{15} \mathrm{CO}-\mathrm{Glu}-\mathrm{D}-\mathrm{Orn}-\mathrm{D}-\mathrm{Ty}$-D-Thr-Glu-D-Ala-Pro-Gln-Tyr-Ile-OH. The hydrolysis of the peptide BPC850 was performed following the methodology described above. $\mathrm{C}_{7} \mathrm{H}_{15} \mathrm{CO}-\mathrm{Glu}-\mathrm{D}-\mathrm{Orn}-\mathrm{D}-\mathrm{Tyr}-\mathrm{D}-$ Thr-Glu-D-Ala-Pro-Gln-Tyr-Ile-OH $\left(t_{R}=6.92 \mathrm{~min}, 35 \%\right.$ HPLC purity) together with the methyl ester derivative $\mathrm{C}_{7} \mathrm{H}_{15} \mathrm{CO}-$ Glu-D-Orn-D-Tyr-D-Thr-Glu-D-Ala-Pro-Gln-Tyr-Ile-OMe $\left(t_{R}=7.12 \mathrm{~min}, 23 \%\right.$ HPLC purity) were obtained; MS (ESI) $m / z(+): 1353.6[\mathrm{M}+\mathrm{H}]^{+}, 1368.6[\mathrm{M}(\mathrm{OMe})+\mathrm{H}]^{+}, 1375.7$ $[\mathrm{M}+\mathrm{Na}]^{+}$; MS (ESI) $m / z(-): 1351.6[\mathrm{M}-\mathrm{H}]^{-}, 1365.6[\mathrm{M}(\mathrm{OMe})-\mathrm{H}]^{-}, 1387.5[\mathrm{M}(\mathrm{OMe})+\mathrm{Na}-2 \mathrm{H}]^{-}$.

$\mathrm{C}_{7} \mathrm{H}_{15} \mathrm{CO}-\mathrm{Glu}-\mathrm{D}-\mathrm{Orn}-\mathrm{D}-\mathrm{Tyr}-\mathrm{D}-\mathrm{Ser}-\mathrm{Glu}-\mathrm{D}-\mathrm{Ala}-\mathrm{Pro}-\mathrm{Gln}-\mathrm{Tyr}-\mathrm{Ile}-\mathrm{OH}$. The hydrolysis of the peptide BPC852 was performed following the methodology described above. $\mathrm{C}_{7} \mathrm{H}_{15} \mathrm{CO}-\mathrm{Glu}$-D-Orn-D-Tyr-DSer-Glu-D-Ala-Pro-Gln-Tyr-Ile-OH $\left(t_{R}=6.87 \mathrm{~min}, 33 \%\right.$ HPLC purity) together with the methyl ester derivative $\mathrm{C}_{7} \mathrm{H}_{15} \mathrm{CO}$-Glu-D-Orn-D-Tyr-D-Ser-Glu-D-Ala-Pro-Gln-Tyr-Ile-OMe $\left(t_{R}=7.07 \mathrm{~min}, 30 \%\right.$ 
HPLC purity) were obtained; MS (ESI) $m / z(+): 1339.7[\mathrm{M}+\mathrm{H}]^{+}, 1353.6[\mathrm{M}(\mathrm{OMe})+\mathrm{H}]^{+}, 1361.7$ $[\mathrm{M}+\mathrm{Na}]^{+} ; \mathrm{MS}(\mathrm{ESI}) \quad m / z(-): 1337.6[\mathrm{M}-\mathrm{H}]^{-}, 1351.7[\mathrm{M}(\mathrm{OMe})-\mathrm{H}]^{-}, 1359.5[\mathrm{M}+\mathrm{Na}-2 \mathrm{H}]^{-}, 1373.5$ $[\mathrm{M}(\mathrm{OMe})+\mathrm{Na}-2 \mathrm{H}]^{-}$

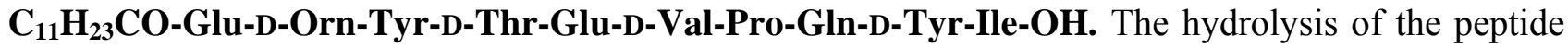
BPC854 was performed following the methodology described above. $\mathrm{C}_{11} \mathrm{H}_{23} \mathrm{CO}$-Glu-D-Orn-Tyr-DThr-Glu-D-Val-Pro-Gln-D-Tyr-Ile-OH $\left(t_{R}=7.99 \mathrm{~min}, 30 \%\right.$ HPLC purity) together with the methyl ester derivative $\mathrm{C}_{11} \mathrm{H}_{23} \mathrm{CO}$-Glu-D-Orn-Tyr-D-Thr-Glu-D-Val-Pro-Gln-D-Tyr-Ile-OMe $\left(t_{R}=8.09\right.$ min, 16\% HPLC purity) were obtained; MS (ESI) $m / z(+): 726.4[\mathrm{M}(\mathrm{OMe})+2 \mathrm{H}]^{2+}, 1437.8[\mathrm{M}+\mathrm{H}]^{+}, 1451.8$ $\left[\mathrm{M}(\mathrm{OMe})+\mathrm{H}^{+}\right.$; MS (ESI) m/z(-): 1435.7 [M-H]', 1449.8 [M(OMe)-H]', $1472.7[\mathrm{M}(\mathrm{OMe})+\mathrm{Na}-2 \mathrm{H}]^{-}$.

$\mathrm{C}_{15} \mathrm{H}_{31}$ CO-Glu-D-Orn-Tyr-D-Thr-Glu-D-Val-Pro-GIn-D-Tyr-Ile-OH. The hydrolysis of the peptide BPC856 was performed following the methodology described above. $\mathrm{C}_{15} \mathrm{H}_{31} \mathrm{CO}-\mathrm{Glu}-\mathrm{D}-\mathrm{Orn}$-Tyr-DThr-Glu-D-Val-Pro-Gln-D-Tyr-Ile-OH $\left(t_{R}=8.96 \mathrm{~min}, 20 \%\right.$ HPLC purity) together with the methyl ester derivative $\mathrm{C}_{15} \mathrm{H}_{31} \mathrm{CO}-\mathrm{Glu}$-D-Orn-Tyr-D-Thr-Glu-D-Val-Pro-Gln-D-Tyr-Ile-OMe $\left(t_{R}=9.05 \mathrm{~min}, 19 \%\right.$ HPLC purity) were obtained; MS (ESI) $m / z(+): 754.9[\mathrm{M}(\mathrm{OMe})+2 \mathrm{H}]^{2+}, 1493.9[\mathrm{M}+\mathrm{H}]^{+}, 1507.9$ $\left[\mathrm{M}(\mathrm{OMe})+\mathrm{H}^{+}\right.$; MS (ESI) m/z(-): $1491.8[\mathrm{M}-\mathrm{H}]^{-}, 1505.8[\mathrm{M}(\mathrm{OMe})-\mathrm{H}]^{-}, 1527.7[\mathrm{M}(\mathrm{OMe})+\mathrm{Na}-2 \mathrm{H}]^{-}$.

$\mathrm{C}_{11} \mathrm{H}_{23} \mathrm{CO}$-Glu-D-Orn-D-Tyr-D-Thr-Glu-D-Val-Pro-GIn-Tyr-Ile-OH. The hydrolysis of the peptide BPC858 was performed following the methodology described above. $\mathrm{C}_{11} \mathrm{H}_{23} \mathrm{CO}-\mathrm{Glu}-\mathrm{D}-\mathrm{Orn}-\mathrm{D}-\mathrm{Tyr}-\mathrm{D}-$ Thr-Glu-D-Val-Pro-Gln-Tyr-Ile-OH $\left(t_{R}=7.92 \mathrm{~min}, 43 \%\right.$ HPLC purity) together with the methyl ester derivative $\mathrm{C}_{11} \mathrm{H}_{23} \mathrm{CO}-\mathrm{Glu}-\mathrm{D}-\mathrm{Orn}-\mathrm{D}-\mathrm{Tyr}-\mathrm{D}-\mathrm{Thr}$-Glu-D-Val-Pro-Gln-Tyr-Ile-OMe $\left(t_{R}=8.05 \mathrm{~min}, 13 \%\right.$ HPLC purity) were obtained; MS (ESI) $m / z(+): 1437.9[\mathrm{M}+\mathrm{H}]^{+}, 1451.9[\mathrm{M}(\mathrm{OMe})+\mathrm{H}]^{+}, 1459.9$ $[\mathrm{M}+\mathrm{Na}]^{+} ; \quad \mathrm{MS}(\mathrm{ESI}) \quad m / z(-): 1435.8[\mathrm{M}-\mathrm{H}]^{-}, 1449.8[\mathrm{M}(\mathrm{OMe})-\mathrm{H}]^{-}, 1457.7[\mathrm{M}+\mathrm{Na}-2 \mathrm{H}]^{-}, 1471.7$ $[\mathrm{M}(\mathrm{OMe})+\mathrm{Na}-2 \mathrm{H}]^{-}$. 
$\mathrm{C}_{15} \mathrm{H}_{31} \mathrm{CO}-\mathrm{Glu}-\mathrm{D}-\mathrm{Orn}-\mathrm{D}-\mathrm{Tyr}-\mathrm{D}-\mathrm{Thr}-\mathrm{Glu}-\mathrm{D}-$ Val-Pro-GIn-Tyr-Ile-OH. The hydrolysis of the peptide BPC860 was performed following the methodology described above. $\mathrm{C}_{15} \mathrm{H}_{31} \mathrm{CO}-\mathrm{Glu}-\mathrm{D}-\mathrm{Orn}-\mathrm{D}-\mathrm{Tyr}-\mathrm{D}-$ Thr-Glu-D-Val-Pro-Gln-Tyr-Ile-OH $\left(t_{R}=8.87 \mathrm{~min}, 38 \%\right.$ HPLC purity) together with the methyl ester derivative $\mathrm{C}_{15} \mathrm{H}_{31} \mathrm{CO}-\mathrm{Glu}$-D-Orn-D-Tyr-D-Thr-Glu-D-Val-Pro-Gln-Tyr-Ile-OMe $\left(t_{R}=8.94 \mathrm{~min}, 19 \%\right.$ HPLC purity) were obtained; MS (ESI) $m / z(+): 1493.9[\mathrm{M}+\mathrm{H}]^{+}, 1507.9[\mathrm{M}(\mathrm{OMe})+\mathrm{H}]^{+}, 1515.8$ $[\mathrm{M}+\mathrm{Na}]^{+}$; MS (ESI) m/z(-): $1491.9[\mathrm{M}-\mathrm{H}]^{-}, 1505.9$ [M(OMe)-H]-, $1513.8[\mathrm{M}+\mathrm{Na}-2 \mathrm{H}]^{-}$.

\section{Supporting Information}

The Supporting Information is available free of charge on the ACS Publications website at DOI: xxx Spectral data of amino acids HPLC and ESI-MS of linear peptides, depsipeptides and lipodepsipeptides HPLC, ESI-MS, HRMS, 1D and 2D NMR of cyclic lipodepsipeptides

\section{Acknowledgements}

C.R. was the recipient of a predoctoral fellowship from the MINECO of Spain. This work was financed by grants AGL2012-39880-C02-02, AGL2015-69876-C2-2-R (MINECO/FEDER, EU) and MPCUdG2016/038. The authors acknowledge the Serveis Tècnics de Recerca of the University of Girona for the mass spectrometry analysis and the NMR experiments. 


\section{References}

(1) Stein, T. Bacillus Subtilis Antibiotics: Structures, Syntheses and Specific Functions. Mol. Microbiol. 2005, 56, 845-857.

(2) Ongena, M.; Jacques, P. Bacillus Lipopeptides: Versatile Weapons for Plant Disease Biocontrol. Trends Microbiol. 2008, 16, 115-125.

(3) Raaijmakers, J. M.; De Bruijn, I.; Nybroe, O.; Ongena, M. Natural Functions of Lipopeptides from Bacillus and Pseudomonas: More than Surfactants and Antibiotics. FEMS Microbiol. Rev. 2010, 34, 1037-1062.

(4) Bionda, N.; Cudic, P. Cyclic Lipodepsipeptides in Novel Antimicrobial Drug Discovery. Croat. Chem. Acta 2011, 84, 315-329.

(5) Sieber, S. A.; Marahiel, M. A. Learning from Nature's Drug Factories : Nonribosomal Synthesis of Macrocyclic Peptides. J. Bacteriol. 2003, 185, 7036-7043.

(6) Sarabia, F.; Chammaa, S.; Sánchez Ruiz, A.; Martín Ortiz, L.; López Herrera, F. J. Chemistry and Biology of Cyclic Depsipeptides of Medicinal and Biological Interest. Curr. Med. Chem. 2004, 11, $1309-1332$.

(7) Vanittanakom, N.; Loeffler, W.; Koch, U.; Jung, G. J. Fengycin- A Novel Antifungal Lipopeptide Antibiotic Produced by Bacillus Subtilis F-29-3. Antibiot. (Tokyo). 1986, XXXIX, 888-901.

(8) Chen, H.; Wang, L.; Su, C. X.; Gong, G. H.; Wang, P.; Yu, Z. L. Isolation and Characterization of Lipopeptide Antibiotics Produced by Bacillus Subtilis. Lett. Appl. Microbiol. 2008, 47, 180-186.

(9) Bie, X.; Lu, Z.; Lu, F. Identification of Fengycin Homologues from Bacillus Subtilis with ESIMS/CID. J. Microbiol. Methods 2009, 79, 272-278. 
(10)Pueyo, M. T.; Bloch, C.; Carmona-Ribeiro, A. M.; di Mascio, P. Lipopeptides Produced by a Soil Bacillus Megaterium Strain. Microb. Ecol. 2009, 57, 367-378.

(11)Pecci, Y.; Rivardo, F.; Martinotti, M. G.; Allegrone, G. LC/ESI-MS/MS Characterisation of Lipopeptide Biosurfactants Produced by the Bacillus Licheniformis V9T14 Strain. J. Mass Spectrom. 2010, 45, 772-778.

(12)Pathak, K. V.; Keharia, H.; Gupta, K.; Thakur, S. S.; Balaram, P. Lipopeptides from the Banyan Endophyte, Bacillus Subtilis K1: Mass Spectrometric Characterization of a Library of Fengycins. J. Am. Soc. Mass Spectrom. 2012, 23, 1716-1728.

(13) Villegas-Escobar, V.; Ceballos, I.; Mira, J. J.; Argel, L. E.; Peralta, S. O.; Romero-Tabarez, M. Fengycin C Produced by Bacillus Subtilis EA-CB0015. J. Nat. Prod. 2013, 76, 503-509.

(14)Sa, R.-B.; An, X.; Sui, J.-K.; Wang, X.-H.; Ji, C.; Wang, C.-Q.; Li, Q.; Hu, Y.-R.; Liu, X. Purification and Structural Characterization of Fengycin Homologues Produced by Bacillus subtilis from Poplar Wood Bark. Australas. Plant Pathol. 2018, 47, 259-268.

(15) Sang-Cheol, L.; Kim, S.-H.; Park, I.-H.; Chung, S.-Y.; Chandra, M. S.; Yong-Lark, C. Isolation, Purification, and Characterization of Novel Fengycin S from Bacillus Amyloliquefaciens LSC04 Degrading-Crude Oil. Biotechnol. Bioprocess Eng. 2010, 15, 246-253.

(16) Schneider, J.; Taraz, K.; Budzikiewicz, H.; Deleu, M.; Thonart, P.; Jacques, P. The Structure of Two Fengycins from Bacillus Subtilis S499. Zeitschrift fur Naturforsch. - Sect. C J. Biosci. 1999, $54,859-866$.

(17) Nishikiori, T.; Naganawa, H.; Muraoka, Y.; Aoyagi, T.; Umezawa, H. Plipastatins: New Inhibitors of Phospholipase A2, Produced by Bacillus Cereus BMG302-fF67. III. Structural Elucidation of Plipastatins. J. Antibiot. (Tokyo). 1986, XXXIX, 755-761.

(18) Volpon, L.; Besson, F.; Lancelin, J. NMR Structure of Antibiotics Plipastatins A and B from Bacillus Subtilis Inhibitors of Phospholipase A2. FEBS Lett. 2000, 485, 76-80. 
(19)Honma, M.; Tanaka, K.; Konno, K.; Tsuge, K.; Okuno, T.; Hashimoto, M. Termination of the Structural Confusion between Plipastatin A1 and Fengycin IX. Bioorg. Med. Chem. 2012, 20, $3793-3798$.

(20) Ma, Z.; Hu, J. Plipastatin A1 Produced by a Marine Sediment-Derived Bacillus amyloliquefaciens SH-B74 Contributes to the Control of Gray Mold Disease in Tomato. 3 Biotech 2018, 8:125.

(21) Deleu, M.; Paquot, M.; Nylander, T. Effect of Fengycin, a Lipopeptide Produced by Bacillus Subtilis, on Model Biomembranes. Biophys. J. 2008, 94, 2667-2679.

(22)Zhao, H.; Shao, D.; Jiang, C.; Shi, J.; Li, Q.; Huang, Q.; Rajoka, M. S. R.; Yang, H.; Jin, M. Biological activity of lipopeptides from Bacillus. Appl. Microbiol. Biotechnol. 2017, 101, 59515960.

(23) Sur, S.; Romo, T. D.; Grossfield, A. Selectivity and Mechanism of Fengycin, an Antimicrobial Lipopeptide, from Molecular Dynamics. J. Phys. Chem. B 2018, 122, 2219-2226.

(24) Ongena, M.; Jacques, P.; Touré, Y.; Destain, J.; Jabrane, A.; Thonart, P. Involvement of FengycinType Lipopeptides in the Multifaceted Biocontrol Potential of Bacillus Subtilis. Appl. Microbiol. Biotechnol. 2005, 69, 29-38

(25) Ongena, M.; Jourdan, E.; Adam, A.; Paquot, M.; Brans, A.; Joris, B.; Arpigny, J.-L.; Thonart, P. Surfactin and Fengycin Lipopeptides of Bacillus Subtilis as Elicitors of Induced Systemic Resistance in Plants. Environ. Microbiol. 2007, 9, 1084-1090.

(26)Emmert, E. A. B.; Handelsman, J. Biocontrol of Plant Disease: A (Gram-) Positive Perspective. FEMS Microbiol. Lett. 1999, 171, 1-9.

(27)Li, X.-Y.; Mao, Z.-C.; Wang, Y.-H.; Wu, Y.-X.; He, Y.-Q.; Long, C.-L. Diversity and Active Mechanism of Fengycin-Type Cyclopeptides from Bacillus subtilis XF-1 Against Plasmodiophora brassicae. J. Microbiol. Biotechnol. 2013, 23, 313-321. 
(28) Samel, S. A.; Wagner, B.; Marahiel, M. A.; Essen, L.-O. The Thioesterase Domain of the Fengycin Biosynthesis Cluster: A Structural Base for the Macrocyclization of a Non-Ribosomal Lipopeptide J. Mol. Biol. 2006, 359, 876-889.

(29) Sieber, S. A.; Tao, J.; Walsh, C. T.; Marahiel, M. A. Peptidyl thiophenols as substrates for nonribosomal peptide cyclases. Angew. Chem. Int. Ed. 2004, 43, 493-498.

(30)Hur, J.; Jang, J.; Sim, J.; Son, W. S.; Ahn, H.-C;. Kim, T. S.; Shin, Y.-H.; Lim, C.; Lee, S.; An, H.; Kim, S.-H.; Oh, D.-C.; Jo, E.-K.; Jang, J.; Lee, J.; Suh, Y.-G. Conformation-Enabled Total Syntheses of Ohmyungsamycins A and B and Structural Revision of Ohmyungsamycin B. Angew. Chem. Int. Ed. 2018, 57, 3069-3073.

(31)Liu, J.; Zhu, X.; Kim, S. J.; Zhang, W. Antimycin-type depsipeptides: discovery, biosynthesis, chemical synthesis, and bioactivities. Nat. Prod. Rep. 2016, 33, 1146-1165.

(32) Guo, C.; Mandalapu, D.; Ji, X.; Gao, J.; Zhang, Q. Chemistry and Biology of Teixobactin. Chem. Eur. J. 2018, 24, 5406-5422.

(33)Cruz, L. J.; Francesch, A.; Cuevas, C.; Albericio, F. Synthesis and Structure-Activity Relationship of Cytotoxic Marine Cyclodepsipeptide IB 01212 Analogues. ChemMedChem 2007, 2, 10761084.

(34)Hall, E. A.; Kuru, E.; VanNieuwenhze, M. S. Solid-Phase Synthesis of Lysobactin (Katanosin B): Insights into Structure and Function. Org. Lett. 2012, 14, 2730-2733.

(35)Rosés, C.; Camó, C.; Vogels, K.; Planas, M.; Feliu, L. Solid-Phase Synthesis of Cyclic Depsipeptides Containing a Tyrosine Phenyl Ester Bond. Org. Lett. 2016, 18, 4140-4143.

(36) Isidro-Llobet, A.; Guasch-Camell, J.; Álvarez, M.; Albericio, F. p Nitrobenzyloxycarbonyl (pNZ) as a Temporary N $\alpha$ Protecting Group in Orthogonal Solid Phase Peptide Synthesis - Avoiding Diketopiperazine and Aspartimide Formation. Eur. J. Org. Chem. 2005, 3031-3039. 
(37) Grant, G. A. Synthetic Peptides. A User's Guide; W. H. Freeman and Company: United States of America, 1992.

(38) Adamson, J. G.; Hoang, T.; Crivici, A.; Lajoie, G. A. Use of Marfey's Reagent to Quantitate Racemization upon Anchoring of Amino Acids to Solid Supports for Peptide Synthesis. Anal. Biochem. 1992, 202, 210-214.

(39) Gracia, C.; Isidro-llobet, A.; Cruz, L. J.; Acosta, G. A.; Mercedes, A.; Cuevas, C.; Giralt, E.; Albericio, F. Convergent Approaches for the Synthesis of the Antitumoral Peptide, Kahalalide F . Study of Orthogonal Protecting Groups. J. Org. Chem. 2006, 71, 7196-7204.

(40)Kuroda, J.; Fukai, T.; Nomura, T. Collision-Induced Dissociation of Ring-Opened Cyclic Depsipeptides with a Guanidino Group by Electrospray Ionization/ion Trap Mass Spectrometry. $J$. Mass Spectrom. 2001, 36, 30-37.

(41) Stawikowski, M.; Cudic, P. A Novel Strategy for the Solid-Phase Synthesis of Cyclic Lipodepsipeptides. Tetrahedron Lett. 2006, 47, 8587-8590.

(42)Cochrane, J. R.; Yoon, D. H.; McErlean, C. S. P.; Jolliffe, K. a. A Macrolactonization Approach to the Total Synthesis of the Antimicrobial Cyclic Depsipeptide LI-F04a and Diastereoisomeric Analogues. Beilstein J. Org. Chem. 2012, 8, 1344-1351.

(43) Parts of this manuscript are adapted from the Ph. D. dissertation of Cristina Rosés, Universitat de Girona, 2015: Solid-Phase Synthesis of Cell-Penetrating $\gamma$-Peptide/Antimicrobial Peptide Conjugates and of Cyclic Lipodepsipeptides Derived from Fengycins.

(44) Thutewohl, M.; Waldmann, H. Solid-Phase Synthesis of a Pepticinnamin E Library. Bioorg. Med. Chem. 2003, 11, 2591-2615.

(45) Kaiser, E.; Colescott, R. L.; Bossinger, C. D.; Cook, P. Color Test for Detection of Free Terminal Amino Groups in the Solid-Phase Synthesis of Peptides. Anal. Biochem. 1970, 595-598. 
(46) Vojkovsky, T. Detection of Secondary Amines on Solid Phase. Pept. Res. 1995, 8, 236-237. 